\title{
Abnormal Rat Cortical Development Induced by Ventricular Injection of rHMGB1 Mimicked the Pathophysiology of Human Cortical Dysplasia
}

\section{Xiaolin Yang}

Army Medical University

\section{Zhang Xiaoqing}

Army Medical University

\section{Zhongke Wang}

Army Medical University

Guolong Liu

Army Medical University

\section{Kaifeng Shen}

Army Medical University

\section{Gang Zhu}

Army Medical University

\section{Tingting Wang}

Army Medical University

\section{Shengqing LV}

Army Medical University

Chunqing Zhang

Army Medical University

Hui Yang

Army Medical University

Shiyong Liu ( $D$ liushi24252016@163.com )

Second affiliated Hospital, Army Medical University

\section{Research}

Keywords: Cortical dysplasia, High-mobility group box 1, Spike wave, Innate immune inflammation

Posted Date: December 1st, 2020

DOI: https://doi.org/10.21203/rs.3.rs-115999/v1 
License: (c) (i) This work is licensed under a Creative Commons Attribution 4.0 International License. Read Full License 


\section{Abstract}

\section{Background}

Cortical dysplasia (CD) is a common cause of drug-resistant epilepsy. Increasingly, innate immunity is involved in $C D$ with epilepsy. However, it is unclear whether innate immune factors contribute to induce epileptogenic CD. Here, we injected recombinant human HMGB1 (rHMGB1) into the embryonic rat ventricle to determine whether rHMGB1 can induce epileptogenic $\mathrm{CD}$ with pathophysiological characteristics similar to those of human CD.

\section{Methods}

At gestational day 14.5, rHMGB1 was injected into the ventricles of Sprague Dawley (SD) rat embryos. At 2 months postnatal, the effects of rHMGB1 on cortex construction were examined by Nissl staining; the alterations of nerve tissue were detected using immunostaining. At 3 months postnatal, the susceptibility and severity of pilocarpine-induced seizures and the spontaneous epileptic discharges were evaluated by EEG. Open-field tests, novel object recognition tests, and Morris water maze tests were performed to observe the behavior of rHMGB1-treated rats.

\section{Results}

The results showed cortical organization was severely disrupted in the rHMGB1-treated rats, and microgyria and heterotopia also emerged; additionally, disoriented neurons, dysmorphic neurons, and dysplastic neurons were found in the cortical lesions and heterotopias. Subcortical heterotopia also appeared in the white matter and the gray-white junction of the rHMGB1-treated rats. Moreover, the numbers of neurons and astrocytes were increased in the cortical lesions; the neuronal dendrites were thickened, randomly oriented, and frequently crossed each other. Moreover, the immunoreactivity of NR2A, NR2B, NR1, GAD65/67, EAAT1 and EAAT2 indicated that the excitation of cortical lesions and heterotopia were significantly increased. Furthermore, EEG showed more susceptibility and severity of seizures in rHMGB1-treatment rats compared with the control rats. Intriguingly, spontaneous nonepileptic seizure discharges were also detected in the rHMGB1-treated rats after 5 months of age, and spike-wave discharges of approximately $8 \mathrm{~Hz}$ were the most significantly increased synchronously propagated waves throughout the general brain cortex.

\section{Conclusions}

These results indicated that rHMGB1 exposure during pregnancy can modify the cerebral structure of offspring, which results in increased susceptibility to seizures and mimics the pathophysiological characteristics of human CD. Those results suggested that HMGB1 upregulation resulting from various insults could contribute to the development of epileptogenic CD during pregnancy.

\section{Background}


Cortical dysplasia (CD) is a common cause of drug-resistant intractable epilepsy and is detected in 40$50 \%$ of children undergoing epileptic zone resection[1]. However, the pathophysiological mechanism of $\mathrm{CD}$ has remained unknown. Increasing evidence has shown that innate immune inflammation plays an important role in $\mathrm{CD}$ with epilepsy. The inflammatory receptors Toll-like receptor (TLR) and receptor for advanced glycation end products (RAGE) are upregulated in CD and contribute to the high epileptogenicity of $\mathrm{CD}[2]$. Our previous study also indicated that virus infection and TLR-mediated responses are involved in epileptic $\mathrm{CD}[3]$. The above receptors are activated by damage-associated molecular patterns released by impaired tissue[4]. Specifically, high-mobility group box 1 (HMGB1) participates in the generation and amplification of neuroinflammation and promotes the progression of epileptic diseases with or without $C D[4,5]$. However, there is a lack of evidence that HMGB1 and its downstream signals are involved in the pathogenesis that leads to epileptogenic CD.

HMGB1, the key molecule linking tissue damage and stress to innate immune inflammation, is a member of the damage-associated molecular patterns and is widely expressed in the normal cell nucleus[4]. Moreover, HMGB1 is widely present throughout the developing cortex and thalamus in the E14.5 embryonic rodent brain[6]. HMGB1 is restricted to newly generated neurons of the cortical plate, which suggests that HMGB1 plays an important role in brain development[6]. HMGB1 facilitated the spontaneous proliferation, migration, and differentiation of neural stem cells in vitro[7]. HMGB1 also promoted neural progenitor differentiation in the hippocampus of Alzheimer's disease model mice[8]. HMGB1 acts as a macrophage-activating factor by binding to advanced glycation end products (RAGE) and contributes to the proliferation, migration, and sprouting of endothelial cells[9]. The above evidence illustrates a tight relationship between HMGB1 and brain development, and abnormal innate immunity is related to CD development. This suggests that prenatally, increased HMGB1 levels promote the development of $C D$ and may be involved in the pathogenesis of epileptogenic CD. Specifically, when neural cells are stimulated by hypoxia, infection, or other injury factors, HMGB1 is released from dying and necrotic cells[10].

In this study, we examined the effects of rHMGB1 on the developing brain during pregnancy by using Nissl staining and observed neurons, neuronal dendrites, and astrocytes by immunostaining. Moreover, we detected an imbalance in excitation and inhibition in cortical lesions. Furthermore, we monitored the susceptibility of the offspring to epileptic seizures and spontaneous epileptic discharges. We also analyzed the characteristics of raw EEG from the cortex and hippocampus to observe the electrophysiological features of rHMGB1-treated rats. Finally, we analyzed the behavioral changes of rHMGB1-treated rats.

\section{Methods}

\section{Intracerebroventricular injection of rHMGB1 into the rat embryo}


All rats were housed in an environment with a comfortable temperature and humidity on a 12:12 light/dark cycle. Food and water were freely available. As described in a previous study, SD rats carrying pups at 14.5 days of gestation were anesthetized by using pentobarbiturate, and midline laparotomy was performed to expose the uterus[11]. Two microliters of rHMGB1 diluent $(0.1 \mu \mathrm{g}, 0.2 \mu \mathrm{g}$, or $0.5 \mu \mathrm{g} r \mathrm{rMGB} 1)$ was administered to all fetuses via transuterine injection targeting the anterior horn of the lateral ventricle of the right hemisphere of the pup brain. The abdominal incision was sutured after filling with saline. The control rats were injected with the same dose of saline following the same procedure. Then, the rats were allowed to recover in a warm chamber and provided with topical and systemic analgesia.

\section{Tissue preprocessing and Nissl staining}

Rat pups were sacrificed at P60 with pentobarbiturate anesthesia. In the same way, the rats were sacrificed after the completion of EEG recording. All rats were perfused through the left cardiac ventricle with $4 \%$ paraformaldehyde at room temperature after isotonic saline. The brains were postfixed in $4 \%$ paraformaldehyde for 48 hours and then processed into frozen sections and dried at $-20{ }^{\circ} \mathrm{C}$. To observe histological abnormalities, the frozen sections were rehydrated in an ethanol gradient, and then brain tissues were stained by Nissl staining.

\section{Immunohistochemical staining and evaluation}

Frozen sections were subjected to immunostaining. Briefly, the sections were rehydrated in an ethanol gradient, treated with $0.3 \%$ hydrogen peroxide and $0.3 \%$ Triton X-100 for $30 \mathrm{~min}$, and washed three times in PBS. The sections were then incubated at $4{ }^{\circ} \mathrm{C}$ overnight with primary antibodies diluted in PBS containing 5\% normal goat serum (NGS). Primary antibodies against rat NR1 (1:400), NR2A (1:200), NR2B (1:400), GAD65/67 (1:100), GLAST/EAAT1 (1:200), GLT1/EAAT2 (1:400), NeuN (1:500) and GFAP (1:500) were purchased from Abcam in China. On the second day, the sections were washed three times in PBS and incubated with the appropriate horseradish peroxidase-conjugated secondary antibodies (Zhong Shan Jin Qiao, Shanghai, China) for 1 hour at $37^{\circ} \mathrm{C}$. Finally, the sections were washed again in PBS and reacted with 0.02\% 3,3-diaminobenzidine tetrahydrochloride (DAB, Zhong Shan Jin Qiao,

Shanghai, China). All sections were counterstained with hematoxylin for $30 \mathrm{~s}$, dehydrated and coverslipped. The intensity of NR1, NR2A, NR2B, GAD65/67, GLAST/EAAT1, and GLT1/EAAT2 immunoreactivity was semiquantitatively measured using ImageJ software for Windows (National Institutes of Health, U.S.A.). We selected cells or areas from the lesioned and control cortices and the hippocampus of rats from each group. The staining density of each cell body (excluding the nucleus) was measured, and the staining density of normal subcortical white matter was subtracted for standardization.

\section{Placement of electrodes}


Recording electrodes for EEG monitoring were placed on the bilateral frontal cortices, hippocampus, and occipital cortices of the rats. After sedation with pentobarbital, two silver electrodes were inserted into the bilateral hippocampus (AP $-4.7 \mathrm{~mm}, \mathrm{ML} \pm 5.0 \mathrm{~mm}, 5.5 \mathrm{~mm}$ depth from the surface), and the screws were implanted on bilateral frontal cortices (AP $3.0 \mathrm{~mm}, \mathrm{ML} \pm 2.5$ ), bilateral occipital cortices (AP $-7.5 \mathrm{~mm}, \mathrm{ML}$ $\pm 4.0 \mathrm{~mm}$ ), while the reference and ground electrodes were positioned at the olfactory bulb and cerebellum, respectively. All rats were allowed to recover in a warm chamber. Then, the rats were singly housed in a dedicated cage and provided with topical and systemic analgesia and antibiotics.

\section{Seizure behavior evaluation}

Rats received pilocarpine $(0.7 \mathrm{mg} / 5 \mu \mathrm{l})$ via intracerebroventricular injection and were observed to assess the latency of onset seizure and status epilepticus. Seizure scores were evaluated during the next two hours. Behavioral seizure score: stage 1 , akinesia, staring and salivation; stage 2 , head clonus, forelimb clonus, and wet dog shakes; stage 3 , rearing with forelimb clonus; stage 4, rearing and falling with generalized convulsions and status epilepticus; stage 5, death. After 3 hours of status epilepticus, all surviving rats received diazepam intraperitoneally to stop the seizure. Then, the rats were put into a warm chamber to recover and were administered saline intraperitoneally.

\section{Behavior tests}

A All behavioral tests were performed in 3-month-old rats. All experiments were blinded to the laboratory technician. After the tests, all rats were allowed to rest for $24 \mathrm{~h}$. Open-field tests, novel object recognition tests, and Morris water maze tests were performed. The following are brief descriptions of the test methods. Open-field test: Rats were placed in the center of an arena $(60 \times 60 \times 60 \mathrm{~cm})$ and recorded for 10 min using an open-field apparatus (Biowill, Shanghai, China). The total distance, average speed, center active time, and distance and periphery active time were recorded. Novel object recognition test: Before testing, rats were put into the empty testing apparatus for $5 \mathrm{~min}$. During the training, two identical objects were positioned in the right and left corners; the rats were allowed to freely explore both objects for 5 min. After the completion of training, the rats were tested for object memory. After the left top corner object was replaced with a novel object that was a similar size but a different shape and color compared with both previous objects, the rats were put into the center and allowed to explore the objects for $5 \mathrm{~min}$. The time spent exploring the novel and familiar objects was recorded and analyzed. Morris water maze test: This test was performed as described in previous studies. Rats were trained to find a hidden platform in the third quadrant of the pool according to a distal visual cue. The rats were trained for 6 consecutive days at the same time (9:00 PM) each day with 4 quadrant starting points. On day 7 , the probe tests were carried out after the platform was removed.

\section{EEG acquisition and analysis}


Two weeks after surgery, the rHMGB1-treated rats were divided into two groups ( $A$ and B). The EEGs of the control group and group A rHMGB1-treated rats were monitored after pilocarpine administration at 3 months postnatal. Spontaneous discharges and behavioral seizures were monitored for 3 months for 24 hours every day in the B group. The recording settings were a low-frequency filter of $0.1 \mathrm{~Hz}$, a highfrequency filter of $500 \mathrm{~Hz}$, and samples of $3000 \mathrm{~Hz}$. The intensity and frequency distribution of EEG was obtained from the power spectrum by fast Fourier transform with a 2-s Hanning window across a continuous $1-500 \mathrm{~Hz}$ frequency range. The absolute power $(\mu \mathrm{V} 2)$ of each EEG frequency band [delta (1$4 \mathrm{~Hz})$, theta $(4-8 \mathrm{~Hz})$, alpha $(8-13 \mathrm{~Hz})$, beta $(13-30 \mathrm{~Hz})$, lower gamma $(30-60 \mathrm{~Hz})$, higher gamma (60$80 \mathrm{~Hz}$ ), ripple $(80-250 \mathrm{~Hz})$ and fast ripple $(250-500 \mathrm{~Hz})]$ at selected pre-pilocarpine resting periods (background activity) and post-pilocarpine ictal periods were compared between the rHMGB1-treatment rats and the control rats. Time-frequency and wavelet analyses were performed using Morlet wavelets in Brainstorm, which was developed with MATLAB software. The synchrony of spontaneous spike-wave discharges was observed through coherence and cross-correlation analysis by EEGLAB and custom codes.

\section{Statistical methods}

The data are presented as the mean \pm SEM and were plotted by GraphPad 7.0 software. We compared the semiquantitative densitometric data of immunoreactivity intensities between lesions and controls by unpaired t-tests and ANOVAs. The latency of pilocarpine-induced seizures was analyzed using unpaired ttests. We compared the powers of different frequency bands between the control and rHMGB1-treated groups using ANOVAs. Furthermore, the powers of different frequency bands of the spontaneous epileptic discharges were also analyzed by ANOVAs.

\section{Results}

\section{General information}

In some of the ventricle-injected rHMGB1 pups, $23 \%$ (20-30\%) were born dead at $0.2 \mu \mathrm{g} / \mu \mathrm{l}$, with no deaths at $0.1 \mu \mathrm{g} / \mu \mathrm{l}$ but no survival at $0.5 \mu \mathrm{g} / \mu \mathrm{l}$. However, there were no differences in the diet, daily activities, or growth processes, including fur, body weight, and brain weight, between the rHMGB1-treated group and the control group. All animals survived until postnatal day 60 or the completion of EEG recording.

\section{Abnormalities in brain structure}

In this study, Nissl staining showed well-preserved cortical lamination, neuron orientation towards the pia, and normal-sized neurons in the cortex of the control rats (Fig. 1A-C); mild disorganized cortical lamination and obvious six-layered cortices were present in the rats treated with $0.1 \mu \mathrm{g} / \mu \mathrm{lHMGB} 1$, and the neuronal morphology was normal. However, in the group treated with $0.2 \mu \mathrm{g} / \mu \mathrm{rHMGB} 1$, cortical lamination was severely disrupted (Fig. 1D), and cortical lesions and heterotopic cortices were observed 
(Fig. 1D, arrows); intriguingly, linearly-aligned dysplastic neurons that were similar to those observed in FCD la were also found in the cortical lesions (Fig. 1E);furthermore, neuronal morphology and size varied, and the neuronal dendrite numbers were increased (Fig. 1E-F, arrows). Microgyria and heterotopic cortices were also clearly displayed, and even the neural cells in layers II-III were observed to dwell on the V-VI layer which was disappeared (Fig. 1G, arrows); in which the dendrites and size of neurons were increased (Fig. $1 \mathrm{H}-\mathrm{I}$, arrows). The heterotopic nodules in the periventricular region consisted of neurons of variable size, including disoriented neurons, dysmorphic neurons, dysplastic neurons, and balloon-like cells (Fig. $1 \mathrm{~J}-\mathrm{L}$, arrows). Subcortical heterotopia was found in the white matter and the gray-white junction of the rHMGB1-treated rats (Fig. 1M-0). However, enlarged balloon cells and abnormal gross hippocampal abnormalities were not found (Fig. 1P-R).

Neuron-specific nuclear protein (NeuN) immunostaining revealed that the number of neurons was increased, and the neurons of the rHMGB1-treated rats were distributed in a patternless fashion in cortical lesions compared with those of the control rats (Fig. $2 A-C, p<0.01$ ). Glial fibrillary acidic protein (GFAP)positive astrocytes displayed significantly increased cell density in the cortical lesions of the rHMGB1treated rats (Fig. 2D-F, p< 0.0001). Microtubule-associated protein 2 (MAP-2) immunostaining clearly showed that the neuronal dendrites in the lesions were thickened, randomly oriented, and frequently crossed each other (Fig. 2G-H).

\section{Immunohistochemical analysis of neuronal excitation and inhibition}

The neurons in the cortical lesions and heterotopias displayed stronger NR2B immunoreactivity (IR) than those in the control cortices ( $<<0.0001)$, whereas there was significantly stronger NR1 and NR2A IR in the cortical lesions but not in the heterotopias (Fig. 3A-I, p<0.0001). GAD 65/67-positive GABAergic neurons displayed normal cell density in the cortical lesions, but it was mildly reduced in the heterotopias (Fig. 3J$L, p<0.05)$. There was a significant decrease in the strength of EAAT1 IR in the cortical lesions and heterotopia compared with that in the control cortices (Fig. 3Q-T, $p<0.0001$ ). However, there was no significant increase in EAAT2 IR in the cortical lesions, but EAAT2 IR was weaker in the heterotopias than in the cortices of the control rats (Fig. 3U-X, $\mathrm{p}<0.0001$ ). Furthermore, stronger NR2B IR was detected in the entire hippocampal subregion in the rHMGB1-treated group than in the control group; however, stronger NR2A IR was only found in the CA3 and DG of the rHMGB1-treatment group (Fig. 4A-R, $p<0.001$ and $p<0.0001$, respectively).

\section{Pilocarpine-evoked seizure}

After intracerebroventricular injection of pilocarpine, the rats did not move but exhibited increased breathing rates and saliva secretion. In a few minutes, the rats started running with or without whistling. Next, the rats started to exhibit right forelimb tonic extension followed by left forelimb tremors and then 
rearing and falling behavior or bilateral tonic-clonic convulsions. Thus, pilocarpine led to hippocampal seizures in all rats in both groups. The rHMGB1-treated rats displayed a shorter latency of seizure onset and a shorter duration per seizure than the control rats (Fig. 5A-B, $p<0.0001$ ). The incidences of status epilepticus and mortality were also higher in the rHMGB1 treatment group (control, 60\% SE and 30\% mortality; rHMGB1 treatment, $100 \%$ SE and $80 \%$ mortality) (Fig. 5C-D). The behavioral seizure scores were $3 / 5$ in the control group and 4/5 in the rHMGB1-treated group. Therefore, the severity of the seizures triggered by a single pilocarpine dose was significantly higher in the rHMGB1-treated rats than in the control rats. Electroencephalography showed a high voltage spike, particularly in the hippocampus, before seizure onset after pilocarpine administration. Cortical discharges followed the hippocampal seizures. Quantitative measurement of the power spectra showed that the absolute powers of all bands after pilocarpine administration were increased in the cortex and hippocampus of the rHMGB1-treated rats compared with the control rats (Fig. $5 \mathrm{E}-\mathrm{N}, \mathrm{p}<0.0001$ ), and the powers of approximately $4 \mathrm{~Hz}, 8 \mathrm{~Hz}$ and $150 \mathrm{~Hz}$ bands were the most significantly increased in the rHMGB1-treatment rats compared with the control rats, but there was no obvious $8 \mathrm{~Hz}$ peak in the hippocampus (Fig. 5I-L).

\section{Spontaneous epileptic discharges}

We did not record spontaneous epileptic discharges in the rHMGB1-treated rats or in the control rats before 5 months postnatal. Paroxysmal synchronous nonconvulsive spontaneous spike-wave discharges (SWDs) were found in the cortex of $80 \%$ of the rHMGB1-treated rats beyond 5 months postnatal $(n=10)$. Moreover, slow waves appeared before the SWDs (Fig. 6A-C, arrows); the rats did not show abnormal behavior when the SWDs appeared. The time-frequency analysis showed that the SWDs were concentrated in the theta, alpha, and beta bands, and the power spectrum analysis found that the thetaalpha band (approximately $8 \mathrm{~Hz}$ ) was the most significantly increased (Fig. 6A-D, F, p<0.0001). However, the power of the hippocampus was not different from that at baseline (Fig. 6B-E, F).

To examine the temporal and spatial propagation of SWDs, coherence and cross-correlation analyses were employed. The results indicated that the degree of synchrony was the most significant between the frontal cortex and the ipsilateral occipital cortex (Fig. 7A1-A3, F-G, $p<0.001$ ). Intriguingly, synchronization of spontaneous discharges between the remote cortex areas was concentrated at approximately $8 \mathrm{~Hz}$ (Fig. 7A2-E2), suggesting that these slow waves may be involved in the generalization and synchronization of epileptic discharges across various brain cortices.

\section{Behavioral changes}

Open-field tests showed that the rHMGB1-treated rats spent less time in the center than the control rats (Fig. 8A, $p<0.05$ ). The rHMGB1-treated rats also had a decrease in the total distance traveled compared with that of the control rats (Fig. 8B, $p<0.05$ ). The rHMGB1-treated rats also spent less time examining novel objects than the control rats (Fig. $8 \mathrm{C}, \mathrm{p}<0.05$ ). Moreover, the time spent examining the familiar object in object recognition tests was also decreased in the rHMGB1-treated rats compared with the 
control rats (Fig. 8D, p<0.05). The escape latency of the rHMGB1-treated rats was significantly longer than that of the control rats during training on the Morris water maze; the number of platform crosses was also decreased (Fig. 8E-F, p<0.05). These results indicated that the rHMGB1-treated rats were less exploratory and active and even had cognitive deficits and reduced spatial learning and memory.

\section{Discussion}

The present study showed that rHMGB1 exposure on gestational day 14.5 resulted in cortical dysplasia, which consisted of cortical disruption, cortical heterotopia, and dysmorphic and dysplastic neurons. Imbalance of excitation and inhibition emerged in the cortical lesions. In addition, the excitation of the hippocampus was abnormal, although it was morphologically normal. EEG showed the seizures facilitated by pilocarpine administration, even spontaneous epileptic discharges in the rHMGB1-treated rats. Intriguingly, SWDs at approximately $8 \mathrm{~Hz}$ were found to be the most significantly increased in the cortex of 5-month-old rHMGB1-treated rats. Behavioral tests demonstrated that the rHMGB1-treated rats engaged in less exploratory activity and exhibited cognitive deficits and reduced spatial learning and memory. These results suggested that cortical dysplasia was evoked by rHMGB1 preceding seizures and mimicked the pathogenesis of human epileptogenic cortical dysplasia.

\section{The characteristics of cortical dysplasia}

The proper development and organization of the brain require the normal proliferation, migration, and differentiation of cortical neural progenitors. Disruption of these processes lead to CD. In humans, $C D$ is characterized by dyslamination and disrupted cortical architecture, including dysmorphic neurons, dysplastic neurons, and balloon cells, and sometimes abnormal brain size[1]. However, animal models only mimic some $C D$ characteristics, including laminar disorganization, microgyria, focal heterotopia, periventricular heterotopia, and occasionally cytomegalic neurons [12]. Our results showed cortical dyslamination, heterotopia, microgyria, and even dysmorphic and dysplastic neurons, which are characteristics of $\mathrm{CD}$. Increased neuron numbers and neuronal dendrite thickening and crossover and astrogliosis were also observed in the cortical lesions; these features are features of epileptic CD and promote epileptogenesis[13]. Astrogliosis is also associated with injury-induced inflammation and precedes the onset of FCD and TSC seizures[14-16]. These results demonstrated that HMGB1 could be the key factor involved in the development of $C D$.

\section{The feature of various CD models}

To detect the mechanism of CD and CD-associated epilepsy, animal models of CD are essential and are often experimentally induced by physical, chemical, or genetic manipulations in the developing brain[12]. The in utero frozon model mimics microgyria, focal heterotopia, and spontaneous epileptic discharges of the hippocampus[17]; the prenatal irradiation model reproduces microgyria and heterotopia but does not produce spontaneous epileptic discharges. The prenatal intraperitoneal injection of 
methylazoxymethanol (MAM) model exhibits cortical disorganization and periventricular nodules; carmustine 1-3-bis-chloroethyl-nitrosourea (BCNU) was injected intraperitoneally into pregnant rats to mimic laminar disorganization, heterotopias, and cytomegalic neurons, but spontaneous epileptic discharges were not observed in either model[12]. Moreover, the specific gene was manipulated to mimic cortical dysplasia with epilepsy that was caused by a single genetic abnormality $[18,19]$. Our results included cortical disorganization, microgyria, focal heterotopia, and cortical and periventricular heterotopia; some of these were the characteristics of various models and were also features of human $\mathrm{CD}[20]$. Moreover, dysmorphic and dysplastic neurons were also obvious; most importantly, spontaneous epileptic discharges from the cortex were also detected. In addition, the rHMGB1-treated rats had no extensive pathologic changes that were provoked by genetic or general systemic intervention, unlike the TSC knockout, MAM injection, and irradiation models. These results suggested that HMGB1 promoted the development of $C D$ that mimicked the pathogenesis of human $C D$.

\section{Abnormal excitation in CD}

In epileptic CD, dysplastic neurons and dysmorphic neurons are hyperexcitable and could be the pacemaker focus of seizure[21, 22]. In human resected tissues, the N1R immunoreactivity of dysplastic and dysmorphic neurons was increased; moreover, NR2A and NR2B were also overexpressed, which is a feature of hyperexcitable developing neurons[21, 23]. In CD animal models, NR2B and NR1 were also upregulated in lesions[17, 24]. Furthermore, in vitro ifenprodil reduced the excitability of CD brain tissue slices, and in vivo administration of ifenprodil blocked NR2B; these results clarified the important roles of NR2B and NR1 upregulation in increasing epileptogenicity in the rat frozen model of $C D[25,26]$. In this study, NR2B was increased in focal cortical lesions and heterotopia, and NR2A and NR1 were upregulated in the focal cortical lesions, suggesting that excitation was increased and susceptible to seizures.

Studies have shown that GABA neurons are reduced in $C D$, but GABA terminals are increased surrounding dysplastic neurons, and GABA is an excitatory rather than inhibitory neurotransmitter that enhances neuronal synchronization, leading to seizures[27]. In this study, GAD65/67-positive neurons displayed normal cell density in the focal cortical lesions but lower density in the heterotopias. EAAT1 was also downregulated in the focal cortical lesions and heterotopias. Although there was no difference in EAAT2 in the focal cortical lesions, it was reduced in the heterotopias. EAAT1 and EAAT2 are the major transporters involved in the uptake of matrix glutamate[28]. Therefore, the downregulation of EAAT1 and EAAT2 increased matrix glutamate, resulting in the hyperactivity of dysplastic neurons, which could be the mechanism of epileptic discharge in CD. In summary, our results indicated that HMGB1 promoted the development of abnormally excited $C D$, which increased the susceptibility to seizures.

\section{Spike-wave discharges in CD}

Previous studies have shown that spike-wave discharges (SWDs) appear to be in remission before adolescence[29, 30]. However, in our study, SWDs were recorded in adult rats, which suggested that the 
brains of rHMGB1-treated rats could be immature. In the frozen model of CD, SWDs were also detected, were more frequent and had longer durations during slow-wave sleep[30]. The SWD pattern was similar to that of the spontaneous SWDs that have been observed in absence epilepsy and similar to the spike wave that occurs in human absence seizures and electrical status epilepticus[29, 31, 32]. However, it is still unclear whether the mechanism of epileptogenesis is associated with SWDs. Some studies have suggested that evoked epileptiform activities can propagate to almost all cortical areas and could be involved in thalamocortical dysfunction and the imbalance of excitation and inhibition[33]. Therefore, some hypotheses suggest that spike waves and spindles share similar circuit mechanisms[34, 35]. In this study, the EEG displayed a pattern of epileptiform discharges that closely resembled spike waves. A total of $8 / 10$ adult rats exhibited multiple spontaneous nonconvulsive seizure epochs during 24-h intracranial EEG monitoring that were composed of bursts of rhythmic high-amplitude spike waves, and the frequency ranged from 6 to $10 \mathrm{~Hz}$ (theta-alpha band). In contrast, the controls did not display epileptic discharges and seizures $(0 / 10)$. Therefore, theta and alpha band rhythms may play an important role in the formation of spike-wave epileptic discharges. Next, the degree of synchrony was compared between different brain regions through coherence and cross-correlation analysis. Intriguingly, the results revealed that SWDs between the frontal and occipital regions were highly synchronous (distance $10.5 \mathrm{~mm}$ ); in particular, ictal spikes were concentrated to approximately $8 \mathrm{~Hz}$, suggesting that SWDs were highly generalized and that these low frequencies may be involved in generalization and synchronization in different remote brain regions.

\section{The mechanism of HMGB1-evoked CD}

HMGB1 on the neuronal surface and in the extracellular matrix promotes neurite outgrowth, cell migration, and neuroinflammation. In the E14.5 embryonic rodent brain, HMGB1 is widely present throughout the developing cortex and thalamic region, and HMGB1 is restricted to newly generated neurons of the cortical plate[6, 36]. At E16, HMGB1-positive neurons were observed moving to the cortex from the subventricular and ventricular zones[36]. However, beginning at E18, the expression level of HMGB1 was downregulated to the level observed in adults, in which HMGB1 is only present in regions of continuing neurogenesis, such as the hippocampus [36]. HMGB1 has been shown to trigger hippocampal neurogenesis after brain injury[37]. Moreover, HMGB1 knockdown induced a smaller head in zebrafish larvae, which reduced the number of proliferating cells in the neurogenesis zones and the number of neurons in the cortex[38]. Furthermore, HMGB1 injection partially rescued the above phenotype. In addition, cultured cerebellar granule neurons on postnatal days 4 to 7 displayed more extensive neurites and migrated out of the core of cultures in media containing HMGB1. Blocking HMGB1 reduced N18 neuroblastoma and C6 glioma cell migration in a dose-dependent manner[6]. Therefore, in this study, we chose to inject rHMGB1 into the E14.5 embryonic rat brain. The results demonstrated that rHMGB1 increased the number of neurons and promoted cortical neuron disorganization to form focal cortical lesions, microgyria, cortical and periventricular heterotopia, and subcortical heterotopia. Some studies have shown that disorganized superficial cortical layers are a representative feature of FCD la, and 
subcortical heterotopia is a class of $\operatorname{CD}[39,40]$. Therefore, our results suggested that HMGB1 caused epileptic CD that was similar to human CD.

\section{Behavioral abnormalities}

Brain developmental delay and cognitive impairment are common comorbidities of intractable epilepsyassociated $C D$. A previous rat model of $C D$ demonstrated impaired spatial memory, which depends on hippocampal subregions (CA1, CA3, and DG)[41]. It is known that recognition memory relies mostly on cortical areas, whereas some studies have suggested that the hippocampus is not essential for object recognition [42]. Behavioral deficits were observed in the rHMGB1-treated rats, which were similar to reports in the chemical and physical models of cortical dysplasia. The rHMGB1-treated rats were less exploratory and less active, spent more time on novel objects, and had a longer escape latency than the control rats. Thus, our results indicated that rHMGB1 evoked the development of CD and broadly affected the whole brain network to result in cognitive impairment.

\section{Endogenous HMGB1 was the key factor of CD development}

During pregnancy, environmental changes during brain development can result in alterations in cortical organization, such as altered brain size and aberrant neuronal clusters. To date, animal models of CD have mainly depended on physical and chemical damage. Previous studies have demonstrated that chemical and physical insults in the external environment (e.g., MAM and frozen) resulted in the impairment of neurogenesis, the disruption of cell death and apoptosis, the migration of pyramidal neurons, and the tangential migration of interneurons[12]. The above resulting malformations were similar to those observed in resected tissues from patients with CD. However, those treatments were exogenous and dependent on the time and extent of impairment in the developing brain rather than endogenous causes. Furthermore, various insults can induce CD in the pre- or perinatal period, such as hypoxia-ischemia and viral infection, which promote HMGB1 release to amplify inflammation and disrupt cortical development[43]. In conclusion, HMGB1 is the endogenous intersection point of various impairments resulting from chemical, physical and genetic manipulations in the rodent model of $C D$. Therefore, the rHMGB1-treated rats simulated the pathological features of clinical CD resulting from endogenous damage factors.

\section{Conclusion}

Our results showed that HMGB1 could contribute to the process of epileptic CD development, indicating that increased endogenous HMGB1 levels resulting from various insults during pregnancy could evoke the pathogenesis of $C D$.

\section{Declarations}




\section{Ethics Approval}

All research protocols were approved by the local institutional animal care and use committee. All experiments were performed according to the Ethical Guidelines on Animal Experiments and the Internal Animal Care and Use Committee of the Army Medical University. Appropriate manipulation was carried out to minimize the suffering and unnecessary use of animals.

\section{Consent for publication}

Not applicable

\section{Availability of data and materials}

All data in the study were available from the corresponding author on reasonable request.

\section{Competing interests}

The authors declare that they have no competing interests

\section{Funding}

This study is funded by the National Natural Science Foundation of China (No.81771394, No.81371424).

\section{Authors' contribution}

$X L Y$ and $X Q Z$ contributed equally to this study. SYL, HY, and XLY conceived and designed the research. SYL, CQZ, and SQL supervised the experiments. Nissl's staining and IHC were performed by XLY, XQZ, ZKW, GZ, and TTW. The EEG were monitored by XQZ, XLY and KFS. XLY and GLL carried out the EEG analysis and statistical analysis. XLY wrote the first draft and revised it with the suggestions of other authors. SYL reviewed the manuscript and provided advices. All authors have approved to submission of the final manuscript.

\section{Acknowledgements}

We express our appreciation to Bangyun Zhao for the advice for experimental methods and Ying Wu for raising animals.

\section{References}


1. Severino M, Geraldo AF, Utz N, Tortora D, Pogledic I, Klonowski W, Triulzi F, Arrigoni F, Mankad K, Leventer RJ, et al: Definitions and classification of malformations of cortical development: practical guidelines. Brain 2020.doi:10.1093/brain/awaa174

2. Zurolo E, lyer A, Maroso M, Carbonell C, Anink JJ, Ravizza T, Fluiter K, Spliet WG, van Rijen PC, Vezzani A, Aronica E: Activation of Toll-like receptor, RAGE and HMGB1 signalling in malformations of cortical development. Brain 2011, 134:1015-1032.doi:10.1093/brain/awr032

3. Liu S, Lu L, Cheng X, Xu G, Yang H: Viral infection and focal cortical dysplasia. Ann Neuro/ 2014, 75:614-616.doi:10.1002/ana.24037

4. Ravizza T, Terrone G, Salamone A, Frigerio F, Balosso S, Antoine DJ, Vezzani A: High Mobility Group Box 1 is a novel pathogenic factor and a mechanistic biomarker for epilepsy. Brain Behav Immun 2018, 72:14-21.doi:10.1016/j.bbi.2017.10.008

5. Wang F, Yang X, Ma Y, Wei Y, Yang M, Chen X, Chen B, He Q, Yang Q, Yang H, Liu S: TRIF contributes to epileptogenesis in temporal lobe epilepsy during TLR4 activation. Brain Behav Immun 2018, 67:65-76.doi:10.1016/j.bbi.2017.07.157

6. Fang P, Schachner M, Shen YQ: HMGB1 in development and diseases of the central nervous system. Mol Neurobiol 2012, 45:499-506.doi:10.1007/s12035-012-8264-y

7. Wang L, Yu L, Zhang T, Wang L, Leng Z, Guan Y, Wang X: HMGB1 enhances embryonic neural stem cell proliferation by activating the MAPK signaling pathway. Biotechnol Lett 2014, 36:16311639.doi:10.1007/s10529-014-1525-2

8. Meneghini V, Bortolotto V, Francese MT, Dellarole A, Carraro L, Terzieva S, Grilli M: High-mobility group box-1 protein and $\beta$-amyloid oligomers promote neuronal differentiation of adult hippocampal neural progenitors via receptor for advanced glycation end products/nuclear factor-kB axis: relevance for Alzheimer's disease. J Neurosci 2013, 33:6047-6059.doi:10.1523/jneurosci.205212.2013

9. Schlueter C, Weber H, Meyer B, Rogalla P, Roser K, Hauke S, Bullerdiek J: Angiogenetic signaling through hypoxia: HMGB1: an angiogenetic switch molecule. Am J Pathol 2005, 166:12591263.doi:10.1016/s0002-9440(10)62344-9

10. Vijayakumar EC, Bhatt LK, Prabhavalkar KS: High Mobility Group Box-1 (HMGB1): A Potential Target in Therapeutics. Curr Drug Targets 2019, 20:1474-1485.doi:10.2174/1389450120666190618125100

11. Walantus W, Castaneda D, Elias L, Kriegstein A: In utero intraventricular injection and electroporation of E15 mouse embryos. J Vis Exp 2007:239.doi:10.3791/239

12. Luhmann HJ: Models of cortical malformation-Chemical and physical. J Neurosci Methods 2016, 260:62-72.doi:10.1016/j.jneumeth.2015.03.034

13. Kielbinski M, Gzielo K, Soltys Z: Review: Roles for astrocytes in epilepsy: insights from malformations of cortical development. Neuropathol App/ Neurobiol 2016, 42:593606.doi:10.1111/nan.12331

14. Talos DM, Kwiatkowski DJ, Cordero K, Black PM, Jensen FE: Cell-specific alterations of glutamate receptor expression in tuberous sclerosis complex cortical tubers. Ann Neurol 2008, 63:454- 
465.doi:10.1002/ana.21342

15. Sosunov AA, Wu X, Weiner HL, Mikell CB, Goodman RR, Crino PD, McKhann GM, 2nd: Tuberous sclerosis: a primary pathology of astrocytes? Epilepsia 2008, 49 Suppl 2:53-62.doi:10.1111/j.15281167.2008.01493.x

16. Zeng LH, Rensing NR, Zhang B, Gutmann DH, Gambello MJ, Wong M: Tsc2 gene inactivation causes a more severe epilepsy phenotype than Tsc1 inactivation in a mouse model of tuberous sclerosis complex. Hum Mol Genet 2011, 20:445-454.doi:10.1093/hmg/ddq491

17. Kamada T, Sun W, Takase K, Shigeto H, Suzuki SO, Ohyagi Y, Kira J: Spontaneous seizures in a rat model of multiple prenatal freeze lesioning. Epilepsy Res 2013, 105:280-

291.doi:10.1016/j.eplepsyres.2013.03.003

18. Ribierre T, Deleuze C, Bacq A, Baldassari S, Marsan E, Chipaux M, Muraca G, Roussel D, Navarro V, Leguern E, et al: Second-hit mosaic mutation in mTORC1 repressor DEPDC5 causes focal cortical dysplasia-associated epilepsy. J Clin Invest 2018, 128:2452-2458.doi:10.1172/jci99384

19. Lim JS, Gopalappa R, Kim SH, Ramakrishna S, Lee M, Kim WI, Kim J, Park SM, Lee J, Oh JH, et al: Somatic Mutations in TSC1 and TSC2 Cause Focal Cortical Dysplasia. Am J Hum Genet 2017, 100:454-472.doi:10.1016/j.ajhg.2017.01.030

20. Najm IM, Sarnat HB, Blümcke I: Review: The international consensus classification of Focal Cortical Dysplasia - a critical update 2018. Neuropathol App/ Neurobiol 2018, 44:18-

31.doi:10.1111/nan.12462

21. Chassoux F, Devaux B, Landre E, Turak B, Nataf F, Varlet P, Chodkiewicz JP, Daumas-Duport C: Stereoelectroencephalography in focal cortical dysplasia: a 3D approach to delineating the dysplastic cortex. Brain 2000, 123 (Pt 8):1733-1751.doi:10.1093/brain/123.8.1733

22. Cepeda C, Levinson S, Nariai H, Yazon VW, Tran C, Barry J, Oikonomou KD, Vinters HV, Fallah A, Mathern GW, Wu JY: Pathological high frequency oscillations associate with increased GABA synaptic activity in pediatric epilepsy surgery patients. Neurobiol Dis 2020,

134:104618.doi:10.1016/j.nbd.2019.104618

23. Finardi A, Colciaghi F, Castana L, Locatelli D, Marras CE, Nobili P, Fratelli M, Bramerio MA, Lorusso G, Battaglia GS: Long-duration epilepsy affects cell morphology and glutamatergic synapses in type IIB focal cortical dysplasia. Acta Neuropathol 2013, 126:219-235.doi:10.1007/s00401-013-1143-4

24. Deshmukh A, Leichner J, Bae J, Song Y, Valdes-Hernandez PA, Lin WC, Riera JJ: Histological Characterization of the Irritative Zones in Focal Cortical Dysplasia Using a Preclinical Rat Model. Front Cell Neurosci 2018, 12:52.doi:10.3389/fncel.2018.00052

25. Wang X, He X, Li T, Shu Y, Qi S, Luan G: Anti-epileptic effect of ifenprodil on neocortical pyramidal neurons in patients with malformations of cortical development. Exp Ther Med 2017, 14:57575766.doi:10.3892/etm.2017.5311

26. Takase K, Shigeto H, Suzuki SO, Kikuchi H, Ohyagi Y, Kira J: Prenatal freeze lesioning produces epileptogenic focal cortical dysplasia. Epilepsia 2008, 49:997-1010.doi:10.1111/j.1528-

1167.2008.01558.x 
27. Abdijadid S, Mathern GW, Levine MS, Cepeda C: Basic mechanisms of epileptogenesis in pediatric cortical dysplasia. CNS Neurosci Ther 2015, 21:92-103.doi:10.1111/cns.12345

28. Bjørnsen LP, Hadera MG, Zhou Y, Danbolt NC, Sonnewald U: The GLT-1 (EAAT2; slc1a2) glutamate transporter is essential for glutamate homeostasis in the neocortex of the mouse. $J$ Neurochem 2014, 128:641-649.doi:10.1111/jnc.12509

29. Sun QQ, Zhou C, Yang W, Petrus D: Continuous spike-waves during slow-wave sleep in a mouse model of focal cortical dysplasia. Epilepsia 2016, 57:1581-1593.doi:10.1111/epi.13501

30. Williams AJ, Zhou C, Sun QQ: Enhanced Burst-Suppression and Disruption of Local Field Potential Synchrony in a Mouse Model of Focal Cortical Dysplasia Exhibiting Spike-Wave Seizures. Front Neural Circuits 2016, 10:93.doi:10.3389/fncir.2016.00093

31. Coenen AM, Van Luijtelaar EL: Genetic animal models for absence epilepsy: a review of the WAG/Rij strain of rats. Behav Genet 2003, 33:635-655.doi:10.1023/a:1026179013847

32. Antwi P, Atac E, Ryu JH, Arencibia CA, Tomatsu S, Saleem N, Wu J, Crowley MJ, Banz B, Vaca FE, et al: Driving status of patients with generalized spike-wave on EEG but no clinical seizures. Epilepsy Behav 2019, 92:5-13.doi:10.1016/j.yebeh.2018.11.031

33. Luhmann HJ, Raabe K, Qu M, Zilles K: Characterization of neuronal migration disorders in neocortical structures: extracellular in vitro recordings. Eur J Neurosci 1998, 10:3085-3094

34. Beenhakker MP, Huguenard JR: Neurons that fire together also conspire together: is normal sleep circuitry hijacked to generate epilepsy? Neuron 2009, 62:612-632.doi:10.1016/j.neuron.2009.05.015

35. Steriade M: Neuronal substrates of spike-wave seizures and hypsarrhythmia in corticothalamic systems. Adv Neurol 2006, 97:149-154

36. Guazzi S, Strangio A, Franzi AT, Bianchi ME: HMGB1, an architectural chromatin protein and extracellular signalling factor, has a spatially and temporally restricted expression pattern in mouse brain. Gene Expr Patterns 2003, 3:29-33

37. Wang H, Mei X, Cao Y, Liu C, Zhao Z, Guo Z, Bi Y, Shen Z, Yuan Y, Guo Y, et al: HMGB1/Advanced Glycation End Products (RAGE) does not aggravate inflammation but promote endogenous neural stem cells differentiation in spinal cord injury. Sci Rep 2017, 7:10332.doi:10.1038/s41598-01710611-8

38. Zhao X, Kuja-Panula J, Rouhiainen A, Chen YC, Panula P, Rauvala H: High mobility group box-1 (HMGB1; amphoterin) is required for zebrafish brain development. J Biol Chem 2011, 286:2320023213.doi:10.1074/jbc.M111.223834

39. Palmini A, Najm I, Avanzini G, Babb T, Guerrini R, Foldvary-Schaefer N, Jackson G, Lüders HO, Prayson R, Spreafico R, Vinters HV: Terminology and classification of the cortical dysplasias. Neurology 2004, 62:S2-8.doi:10.1212/01.wnl.0000114507.30388.7e

40. Barkovich AJ, Kuzniecky RI, Jackson GD, Guerrini R, Dobyns WB: A developmental and genetic classification for malformations of cortical development. Neurology 2005, 65:18731887.doi:10.1212/01.wnl.0000183747.05269.2d 
41. Clark RE, Broadbent NJ, Squire LR: The hippocampus and spatial memory: findings with a novel modification of the water maze. J Neurosci 2007, 27:6647-6654.doi:10.1523/jneurosci.0913-07.2007

42. Zhou FW, Rani A, Martinez-Diaz H, Foster TC, Roper SN: Altered behavior in experimental cortical dysplasia. Epilepsia 2011, 52:2293-2303.doi:10.1111/j.1528-1167.2011.03267.x

43. Blümcke I, Thom M, Aronica E, Armstrong DD, Vinters HV, Palmini A, Jacques TS, Avanzini G, Barkovich AJ, Battaglia G, et al: The clinicopathologic spectrum of focal cortical dysplasias: a consensus classification proposed by an ad hoc Task Force of the ILAE Diagnostic Methods Commission. Epilepsia 2011, 52:158-174.doi:10.1111/j.1528-1167.2010.02777.x

\section{Figures}




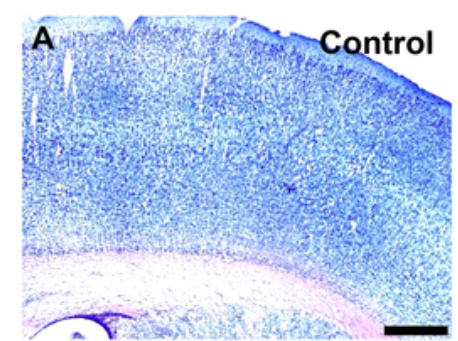

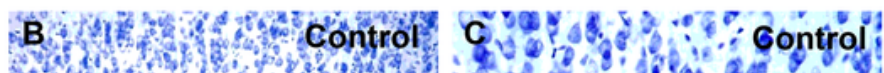
W w w of

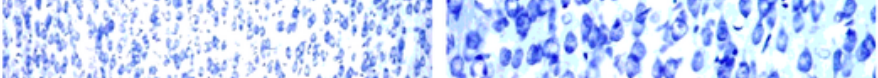

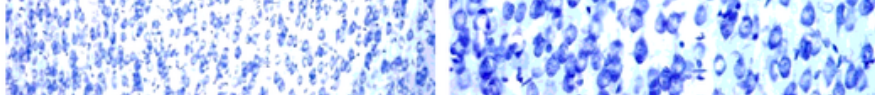
7.

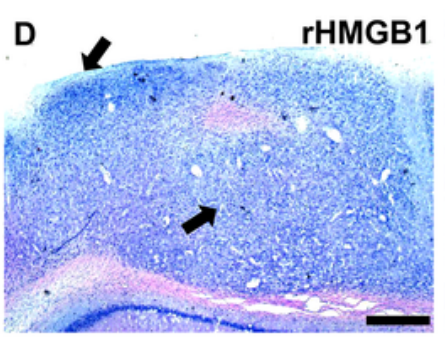

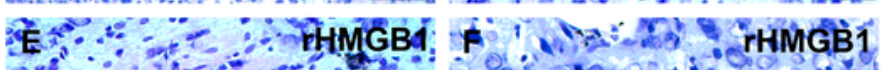
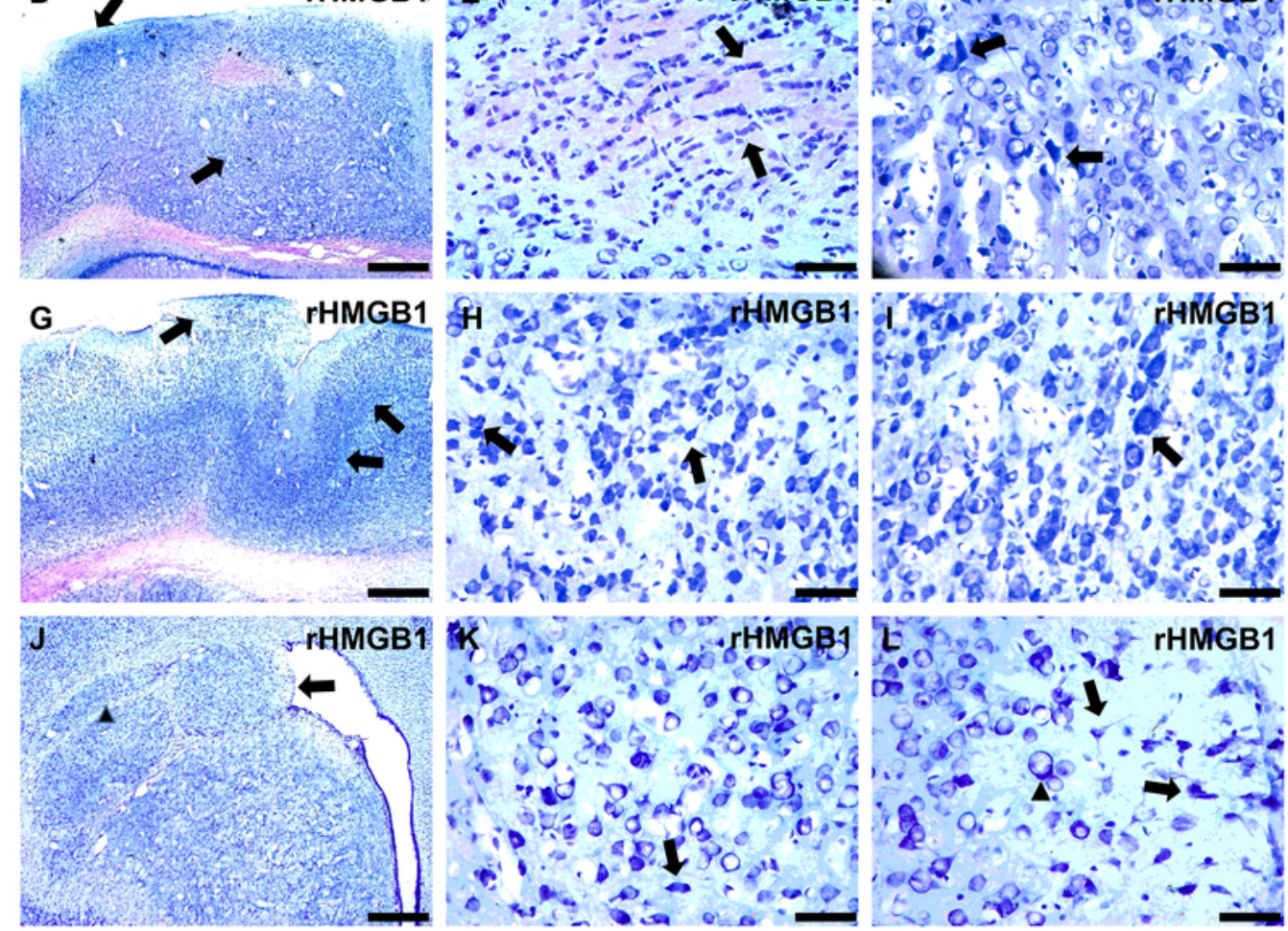

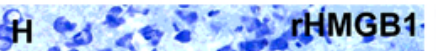

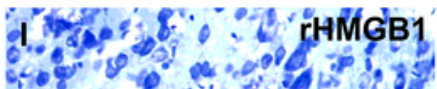
$\therefore 0^{6}-10 \%$
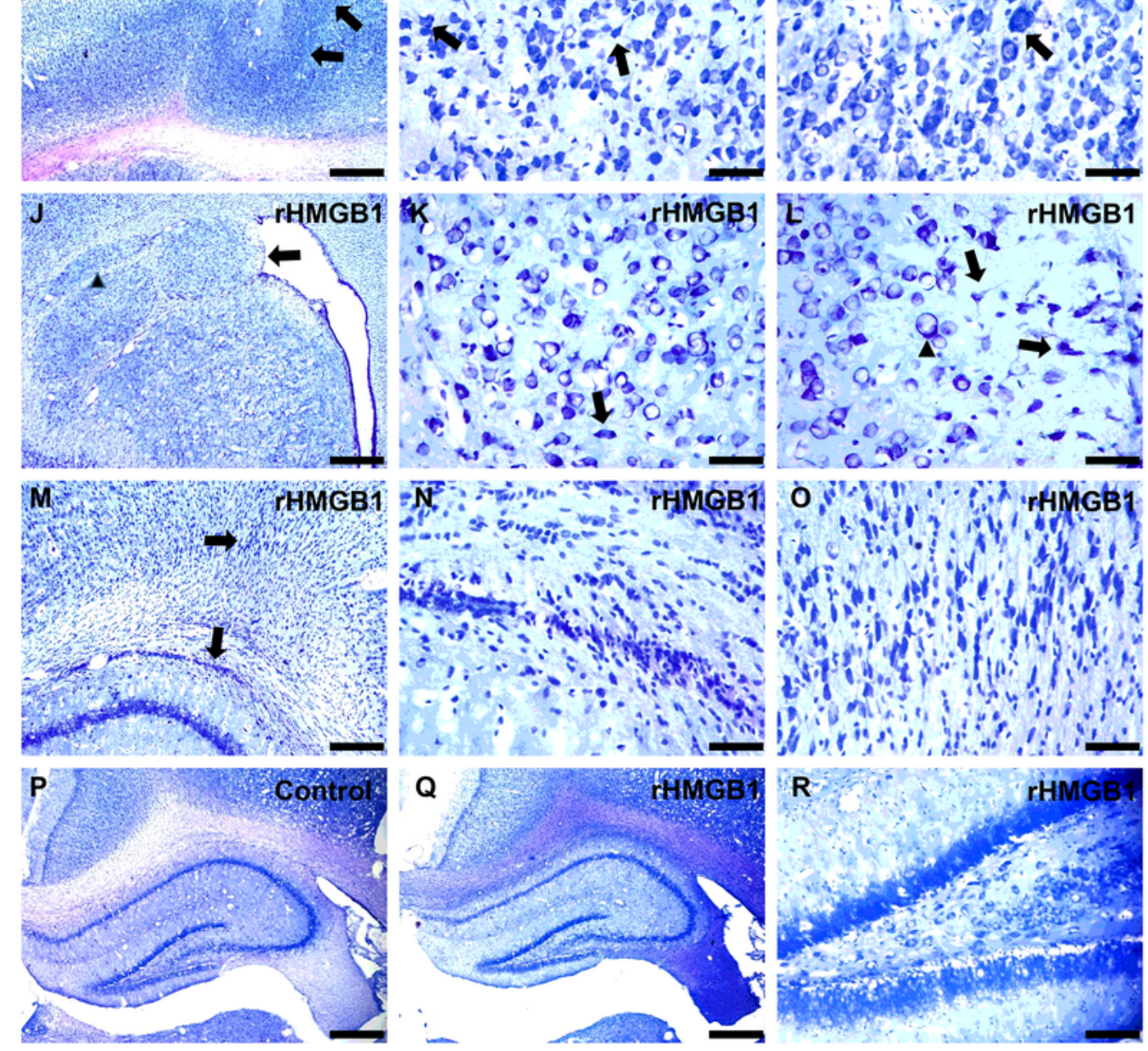

Figure 1

The effects of rHMGB1 during cortex development. A-C: The control showed clear cortex layers and neural orientation; D-F: rHMGB1- treated rats displayed cortical layers disruption, cortical malformation and heterotopic cortex including in disorganized neurons and disoriented neurons (D, F, arrows), even dysplastic neurons which were aligned linearly(E, arrows); G-I: rHMGB1- treated rats showed microgyria and cortex tuber(G, arrows), dysplastic neurons and dendrite increasement (H-I, arrows); J-L: rHMGB1- 
treated rat had paraventricular nodules( $\mathrm{J}$, arrow), subcortical heterotopia( $\mathrm{J}$, triangle), dysmorphic neurons ( $\mathrm{L}$, triangle) and balloon-like cell(K-L, arrows); M-O: The rHMGB1-treated rats displayed neuron migration delayed and retained in the white matter and the grey-white junction (M, arrows); P-R: there were no significant different of the hippocampus between controls and rHMGB1-treated rats in general morphology. rHMGB1, rHMGB1-treated. Scale bars, $500 \mu \mathrm{m}$ for $A, D, G, J, M, P, Q ; 100 \mu m$ for B, R; $50 \mu m$ for the remain.
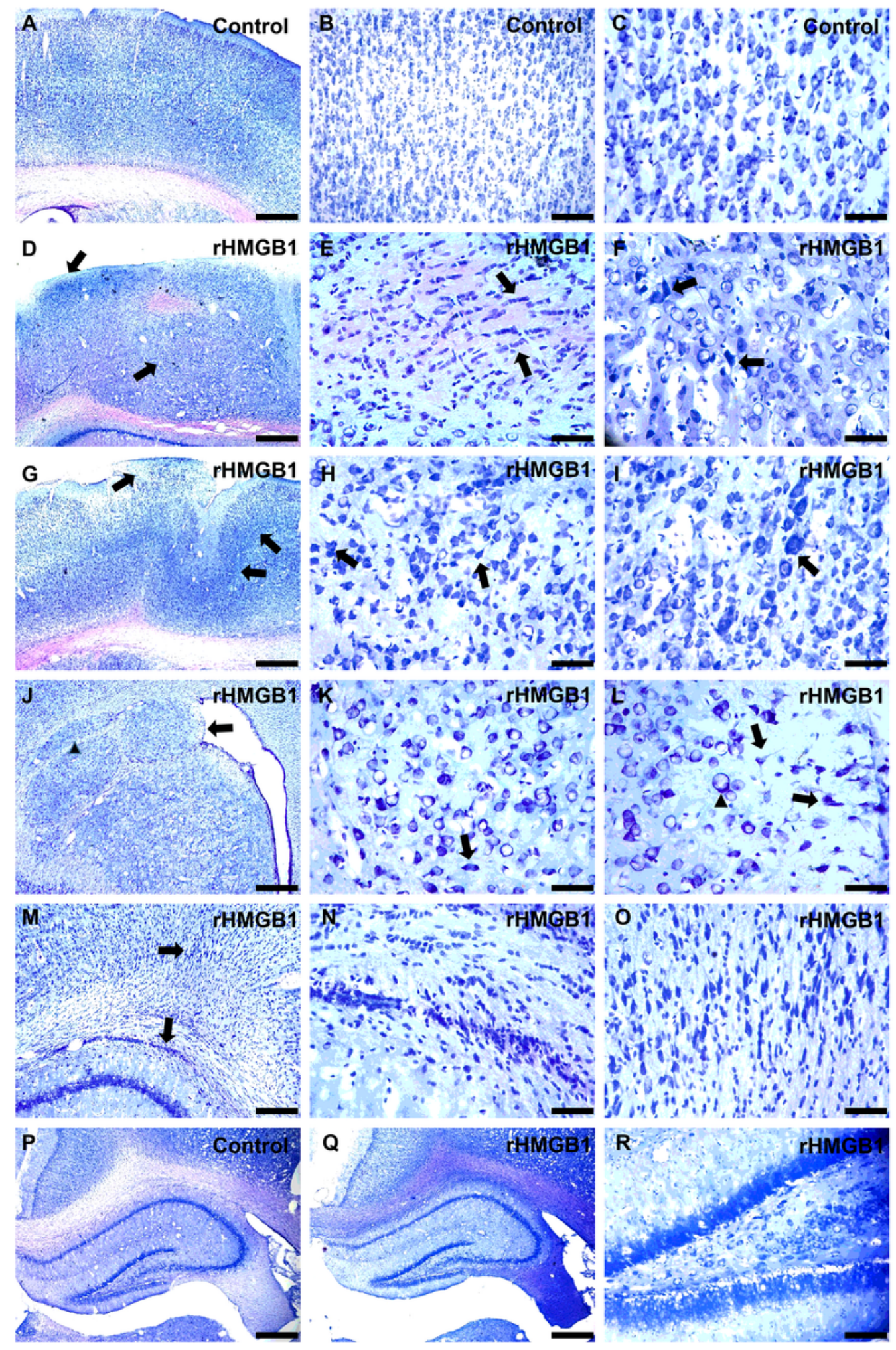

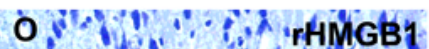

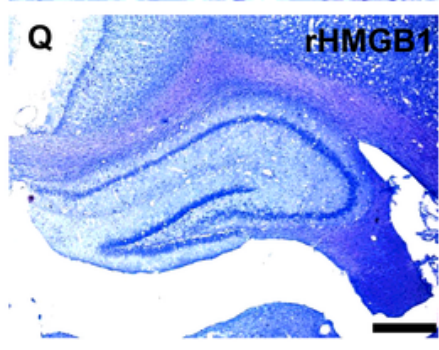

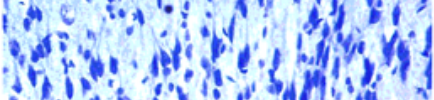
cond

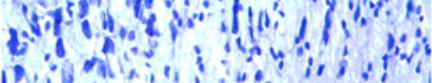

Figure 1 
The effects of rHMGB1 during cortex development. A-C: The control showed clear cortex layers and neural orientation; D-F: rHMGB1- treated rats displayed cortical layers disruption, cortical malformation and heterotopic cortex including in disorganized neurons and disoriented neurons (D, F, arrows), even dysplastic neurons which were aligned linearly(E, arrows); G-I: rHMGB1- treated rats showed microgyria and cortex tuber(G, arrows), dysplastic neurons and dendrite increasement (H-I, arrows); J-L: rHMGB1treated rat had paraventricular nodules( $\mathrm{J}$, arrow), subcortical heterotopia(J, triangle), dysmorphic neurons ( $\mathrm{L}$, triangle) and balloon-like cell(K-L, arrows); M-O: The rHMGB1-treated rats displayed neuron migration delayed and retained in the white matter and the grey-white junction ( $M$, arrows); P-R: there were no significant different of the hippocampus between controls and rHMGB1-treated rats in general morphology. rHMGB1, rHMGB1-treated. Scale bars, $500 \mu \mathrm{m}$ for A, D, G, J, M, P, Q; $100 \mu \mathrm{m}$ for B, R; $50 \mu \mathrm{m}$ for the remain. 


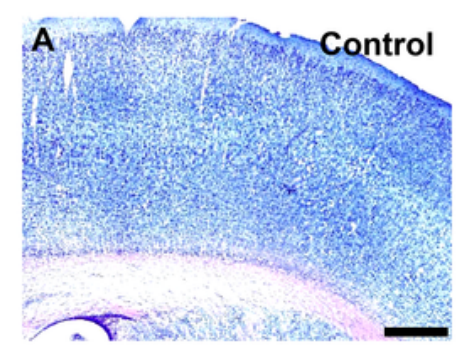

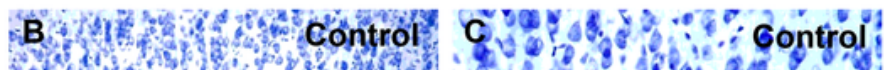
Wow wos 30.

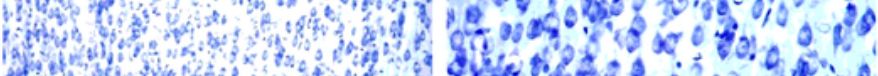
19.

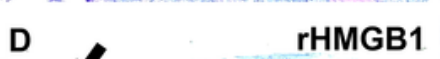

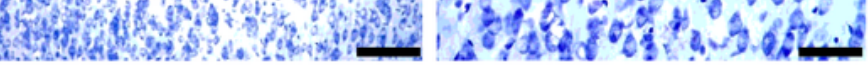
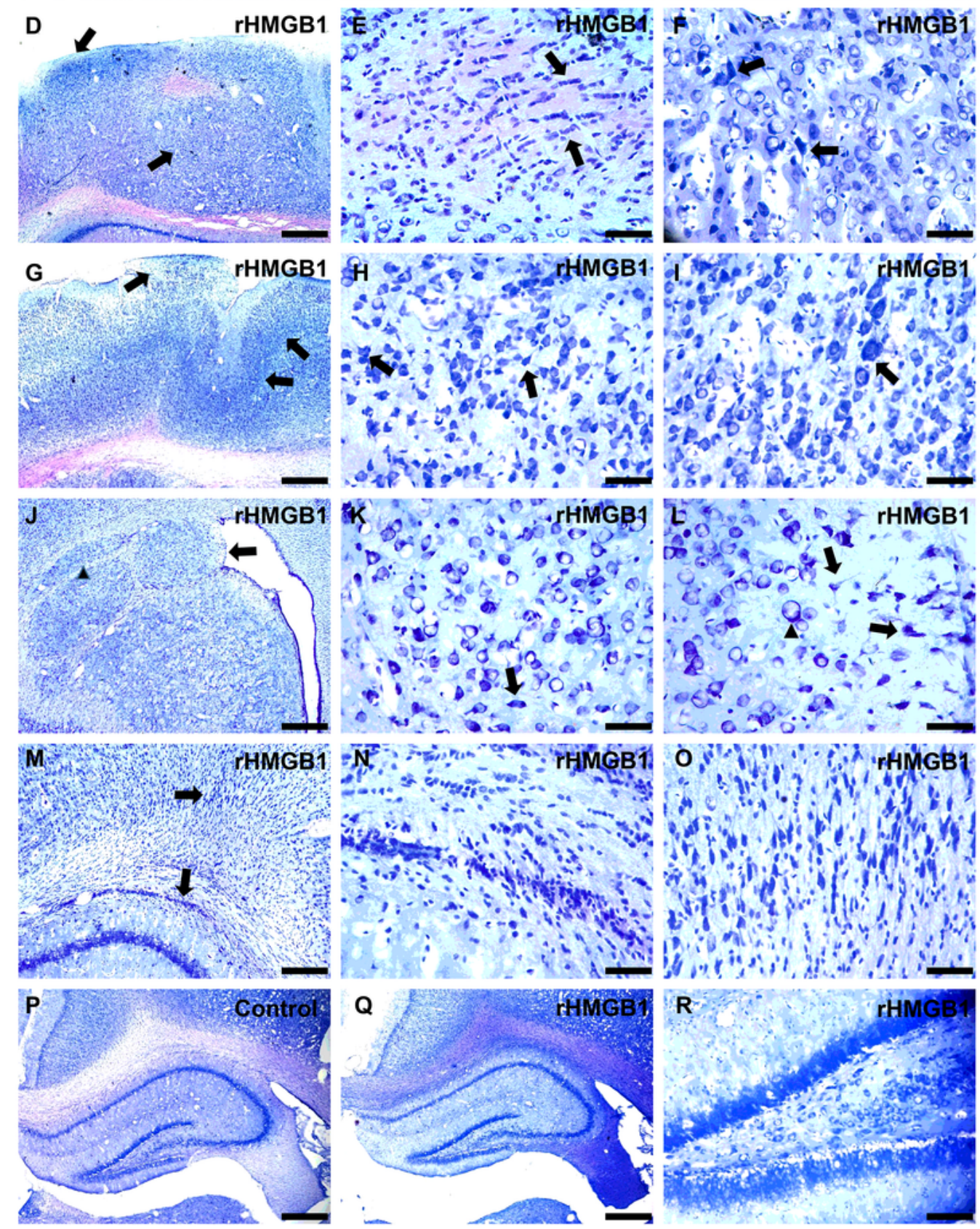

Figure 1

The effects of rHMGB1 during cortex development. A-C: The control showed clear cortex layers and neural orientation; D-F: rHMGB1- treated rats displayed cortical layers disruption, cortical malformation and heterotopic cortex including in disorganized neurons and disoriented neurons (D, F, arrows), even dysplastic neurons which were aligned linearly(E, arrows); G-I: rHMGB1- treated rats showed microgyria and cortex tuber(G, arrows), dysplastic neurons and dendrite increasement (H-I, arrows); J-L: rHMGB1- 
treated rat had paraventricular nodules( $\mathrm{J}$, arrow), subcortical heterotopia( $\mathrm{J}$, triangle), dysmorphic neurons ( $\mathrm{L}$, triangle) and balloon-like cell(K-L, arrows); M-O: The rHMGB1-treated rats displayed neuron migration delayed and retained in the white matter and the grey-white junction (M, arrows); P-R: there were no significant different of the hippocampus between controls and rHMGB1-treated rats in general morphology. rHMGB1, rHMGB1-treated. Scale bars, $500 \mu \mathrm{m}$ for $A, D, G, J, M, P, Q ; 100 \mu m$ for B, R; $50 \mu m$ for the remain.
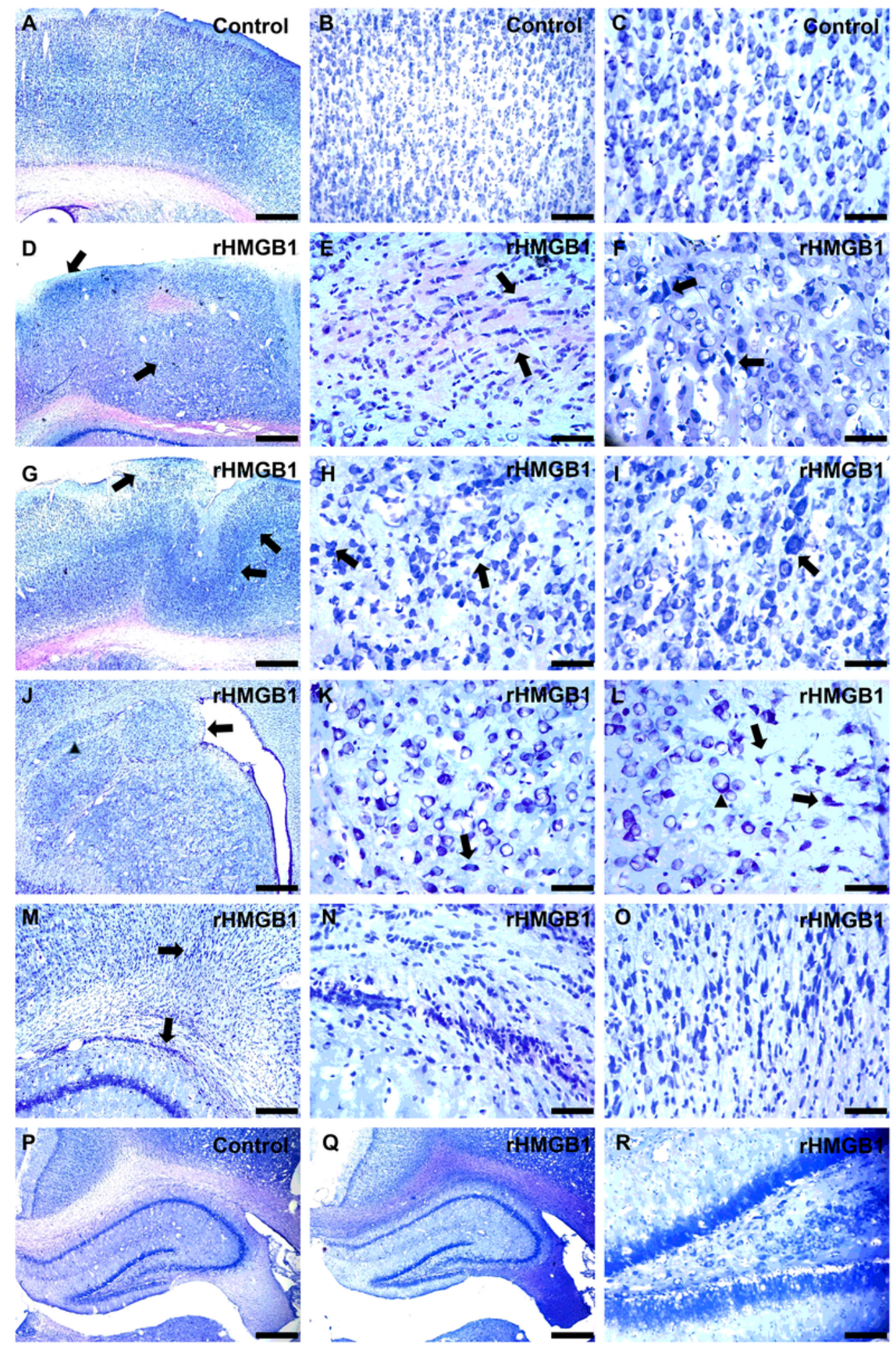

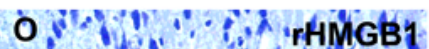

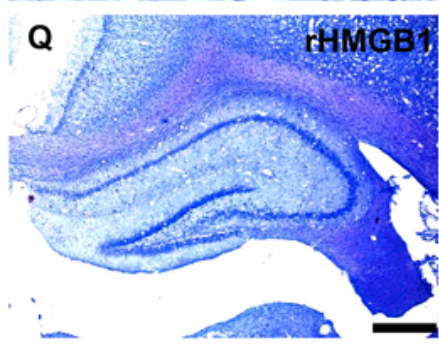

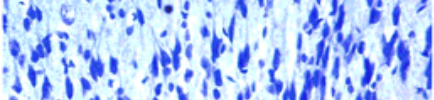
cond

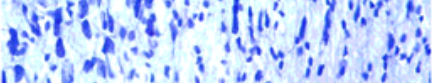

Figure 1 
The effects of rHMGB1 during cortex development. A-C: The control showed clear cortex layers and neural orientation; D-F: rHMGB1- treated rats displayed cortical layers disruption, cortical malformation and heterotopic cortex including in disorganized neurons and disoriented neurons (D, F, arrows), even dysplastic neurons which were aligned linearly(E, arrows); G-I: rHMGB1- treated rats showed microgyria and cortex tuber(G, arrows), dysplastic neurons and dendrite increasement (H-I, arrows); J-L: rHMGB1treated rat had paraventricular nodules $(\mathrm{J}$, arrow), subcortical heterotopia( $\mathrm{J}$, triangle), dysmorphic neurons ( $\mathrm{L}$, triangle) and balloon-like cell(K-L, arrows); M-O: The rHMGB1-treated rats displayed neuron migration delayed and retained in the white matter and the grey-white junction (M, arrows); P-R: there were no significant different of the hippocampus between controls and rHMGB1-treated rats in general morphology. rHMGB1, rHMGB1-treated. Scale bars, $500 \mu \mathrm{m}$ for A, D, G, J, M, P, Q; $100 \mu \mathrm{m}$ for B, R; $50 \mu \mathrm{m}$ for the remain.

\section{Control}
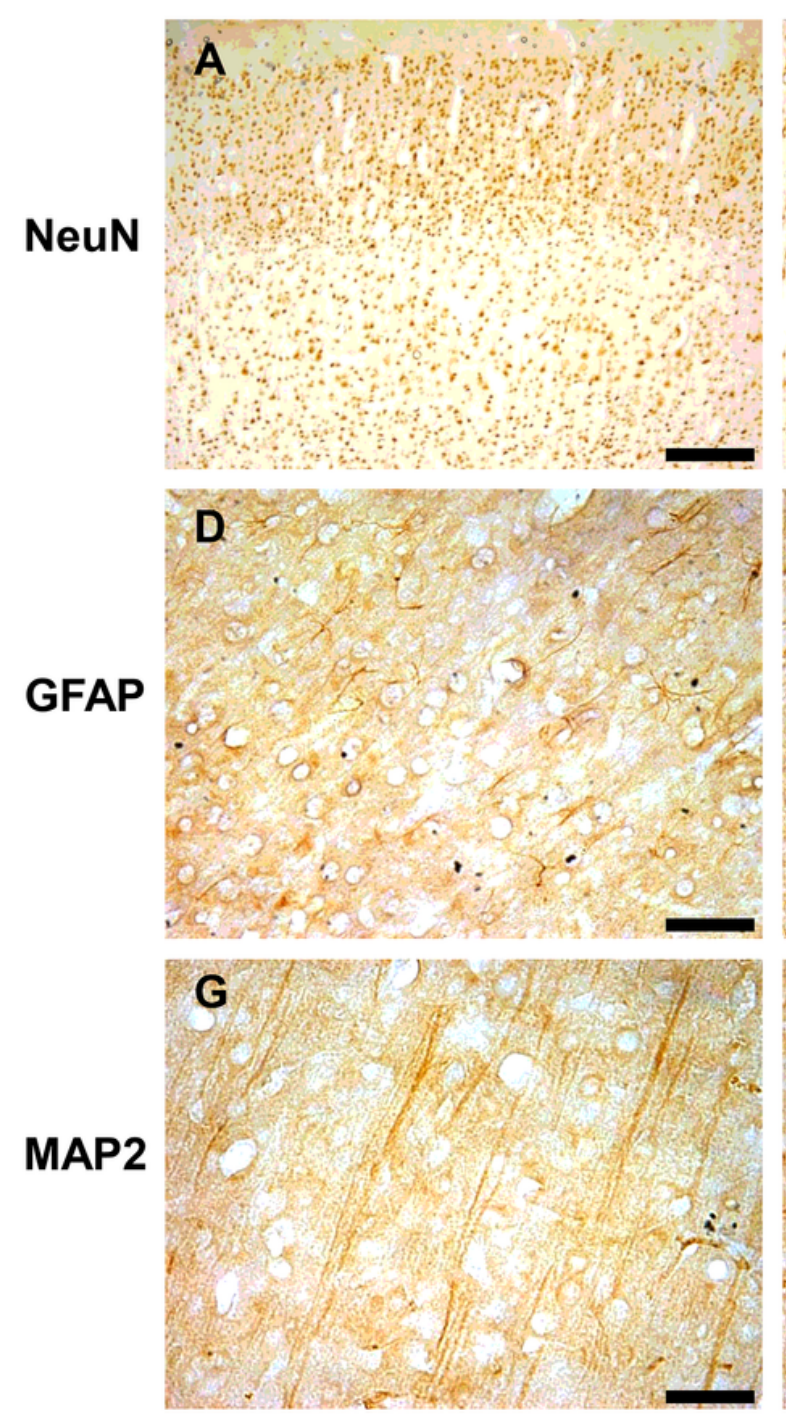

\section{rHMGB1}
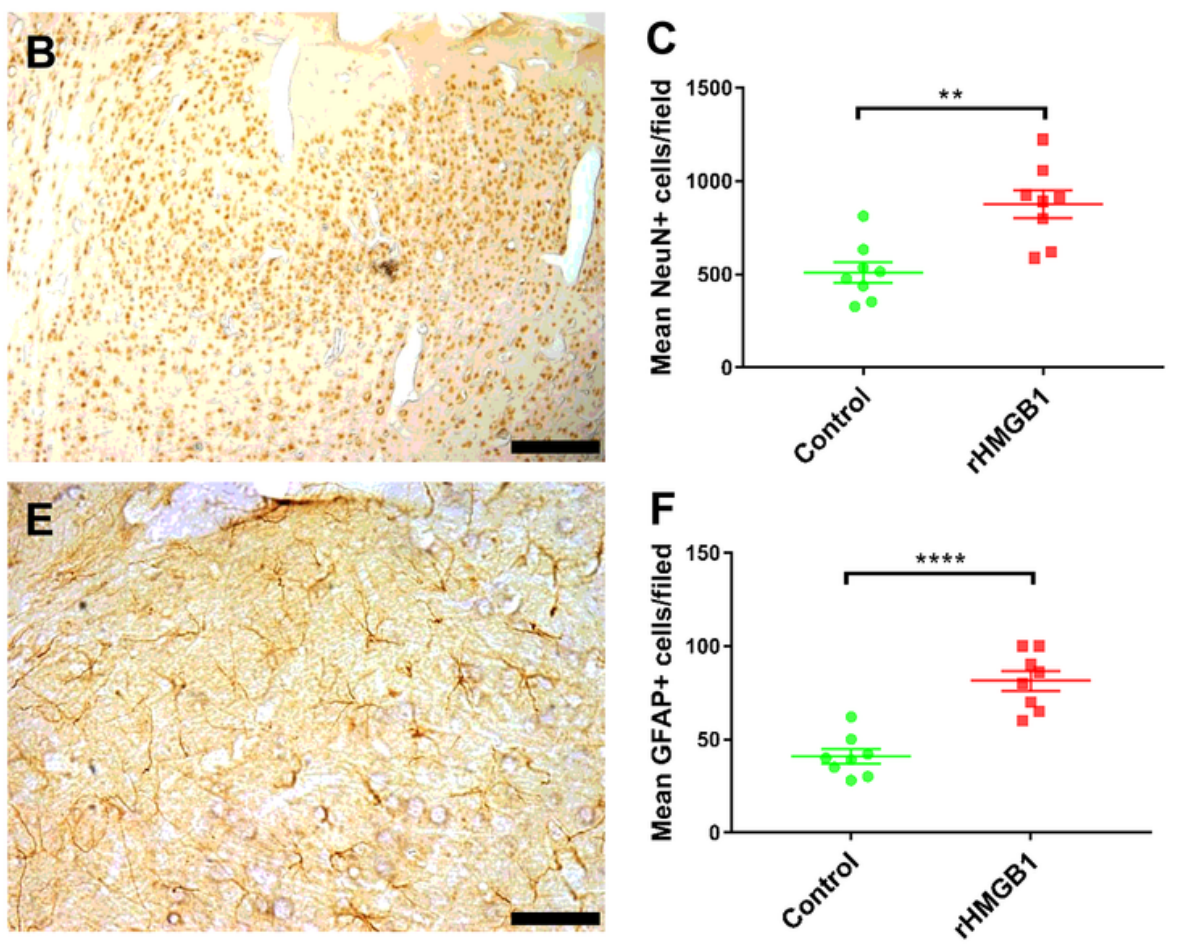

\section{Figure 2}

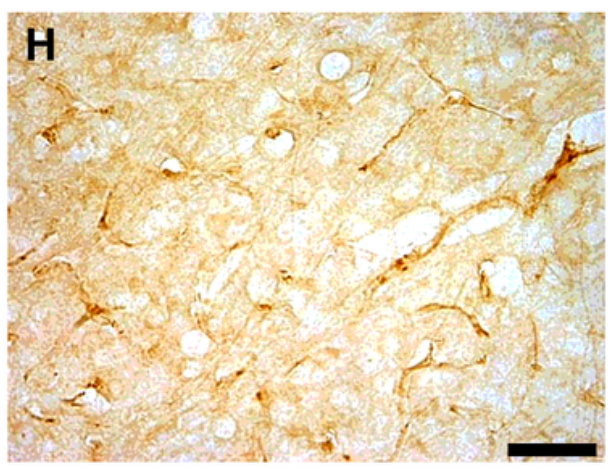

The immunostaining of NeuN, GFAP and MAP2 A-C: The NeuN positive cells were significantly increased in rHMGB1-treated group compared with control group. D-F: GFAP positive astrocyte were also increased 
in the rHMGB1-treated group compared with control group. G-H: The MAP2 staining showed the dendrites randomly oriented and crossed each other, even some dendrites were thicker than the controls. ${ }^{* \star} p<0.01$, ${ }^{* * * *} \mathrm{p}<0.0001$. rHMGB1, rHMGB1-treated. Scale bars, $200 \mu \mathrm{m}$ for A-B; $50 \mu \mathrm{m}$ for $\mathrm{D}-\mathrm{H}$.

\section{Control}

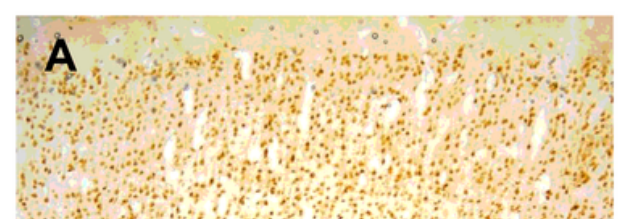

\section{NeuN}

GFAP

MAP2
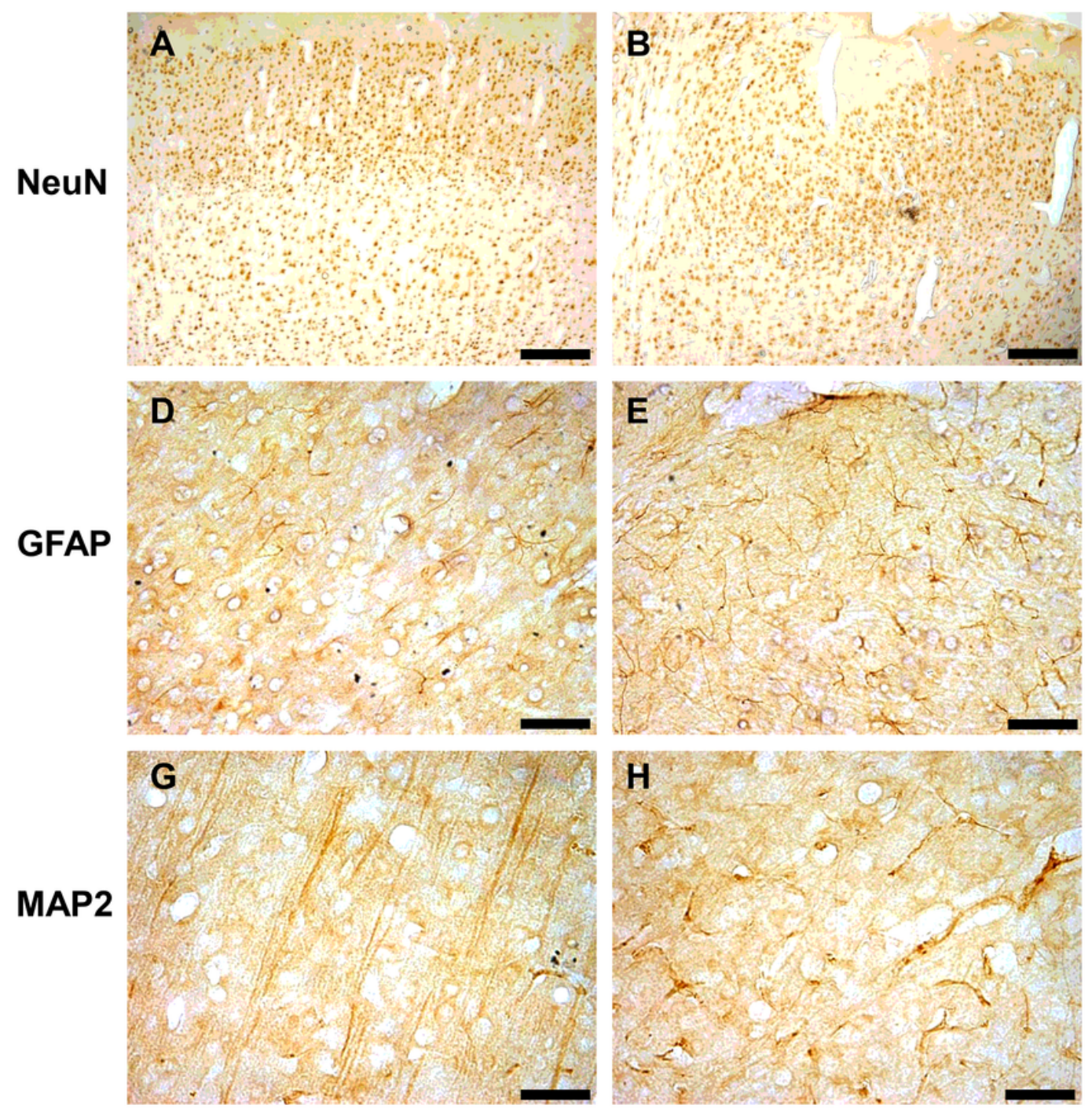

\section{rHMGB1}

C
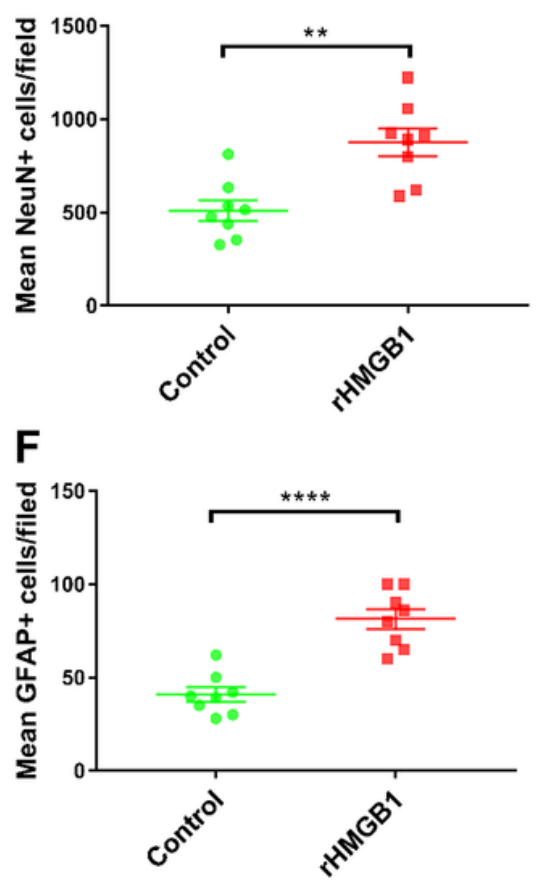

\section{Figure 2}

The immunostaining of NeuN, GFAP and MAP2 A-C: The NeuN positive cells were significantly increased in rHMGB1-treated group compared with control group. D-F: GFAP positive astrocyte were also increased in the rHMGB1-treated group compared with control group. G-H: The MAP2 staining showed the dendrites randomly oriented and crossed each other, even some dendrites were thicker than the controls. ${ }^{* *} p<0.01$, $\star \star \star \star \mathrm{p}<0.0001$. rHMGB1, rHMGB1-treated. Scale bars, $200 \mu \mathrm{m}$ for A-B; $50 \mu \mathrm{m}$ for D-H. 


\section{Control}
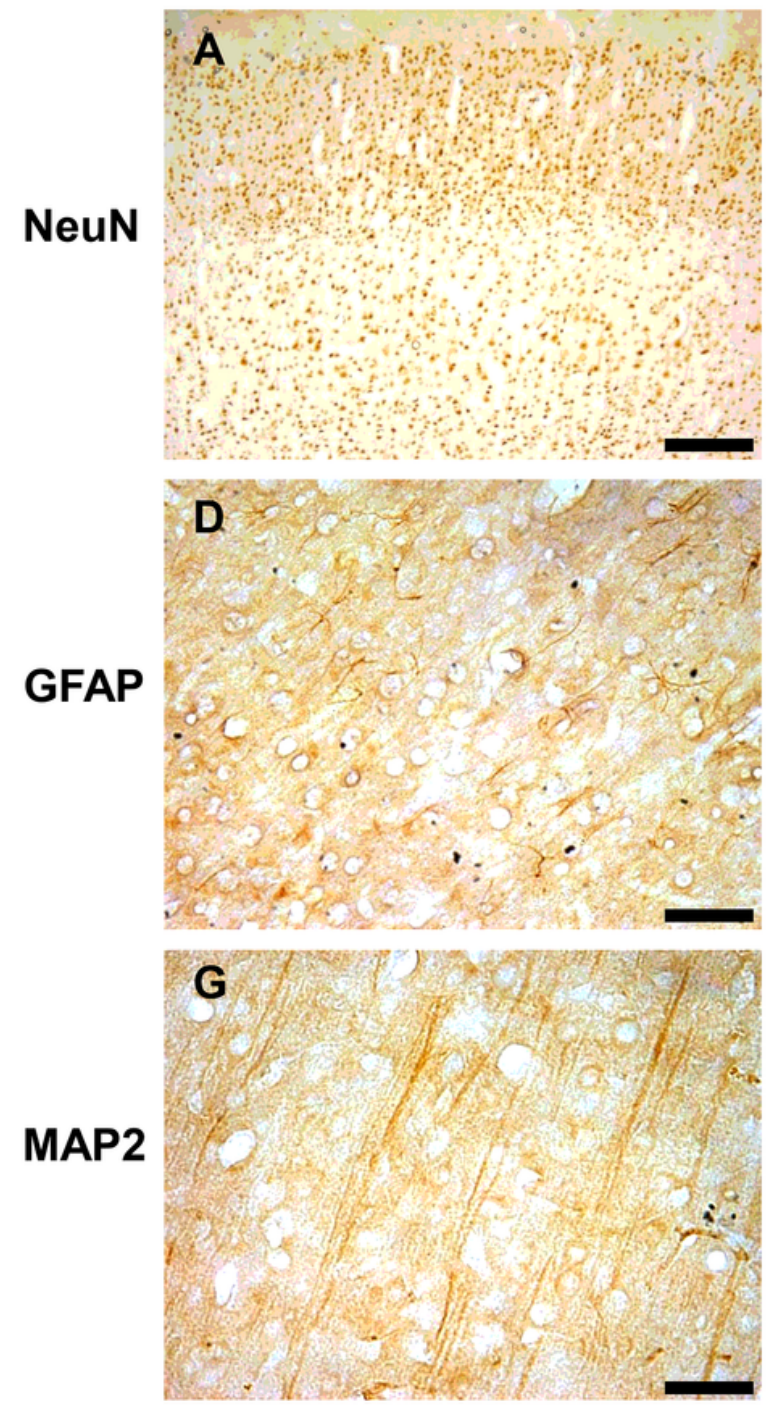

rHMGB1
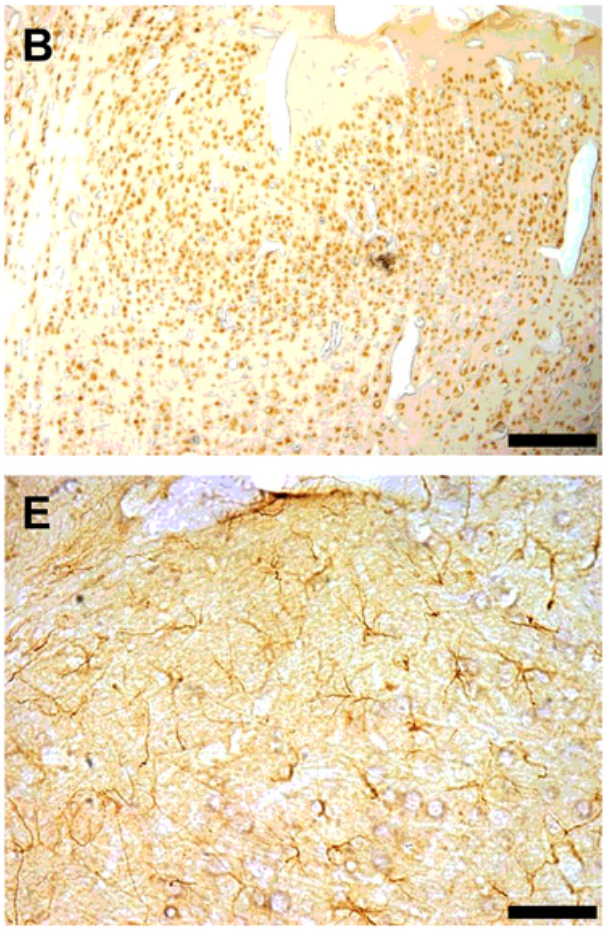

C
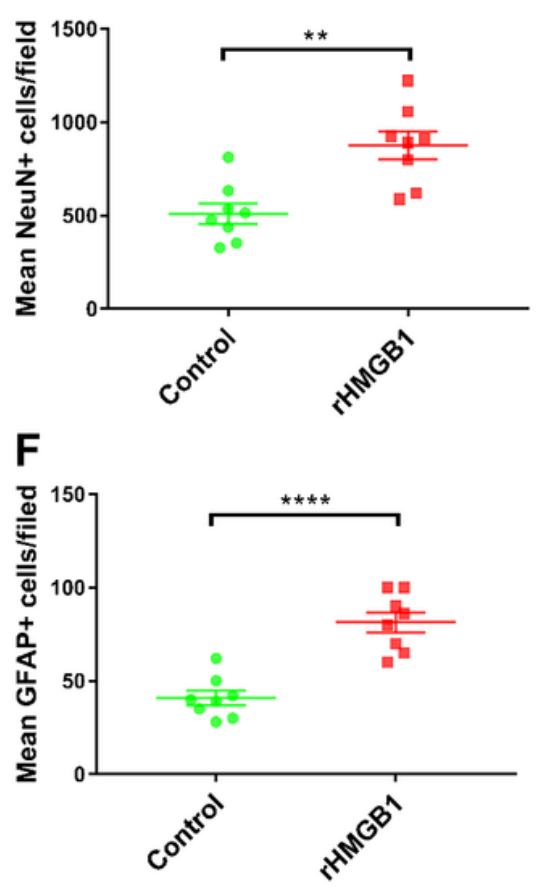

Figure 2

The immunostaining of NeuN, GFAP and MAP2 A-C: The NeuN positive cells were significantly increased in rHMGB1-treated group compared with control group. D-F: GFAP positive astrocyte were also increased in the rHMGB1-treated group compared with control group. G-H: The MAP2 staining showed the dendrites randomly oriented and crossed each other, even some dendrites were thicker than the controls. ${ }^{* *} \mathrm{p}<0.01$, $\star \star \star \star p<0.0001$. rHMGB1, rHMGB1-treated. Scale bars, $200 \mu \mathrm{m}$ for A-B; $50 \mu \mathrm{m}$ for $\mathrm{D}-\mathrm{H}$. 


\section{Control}
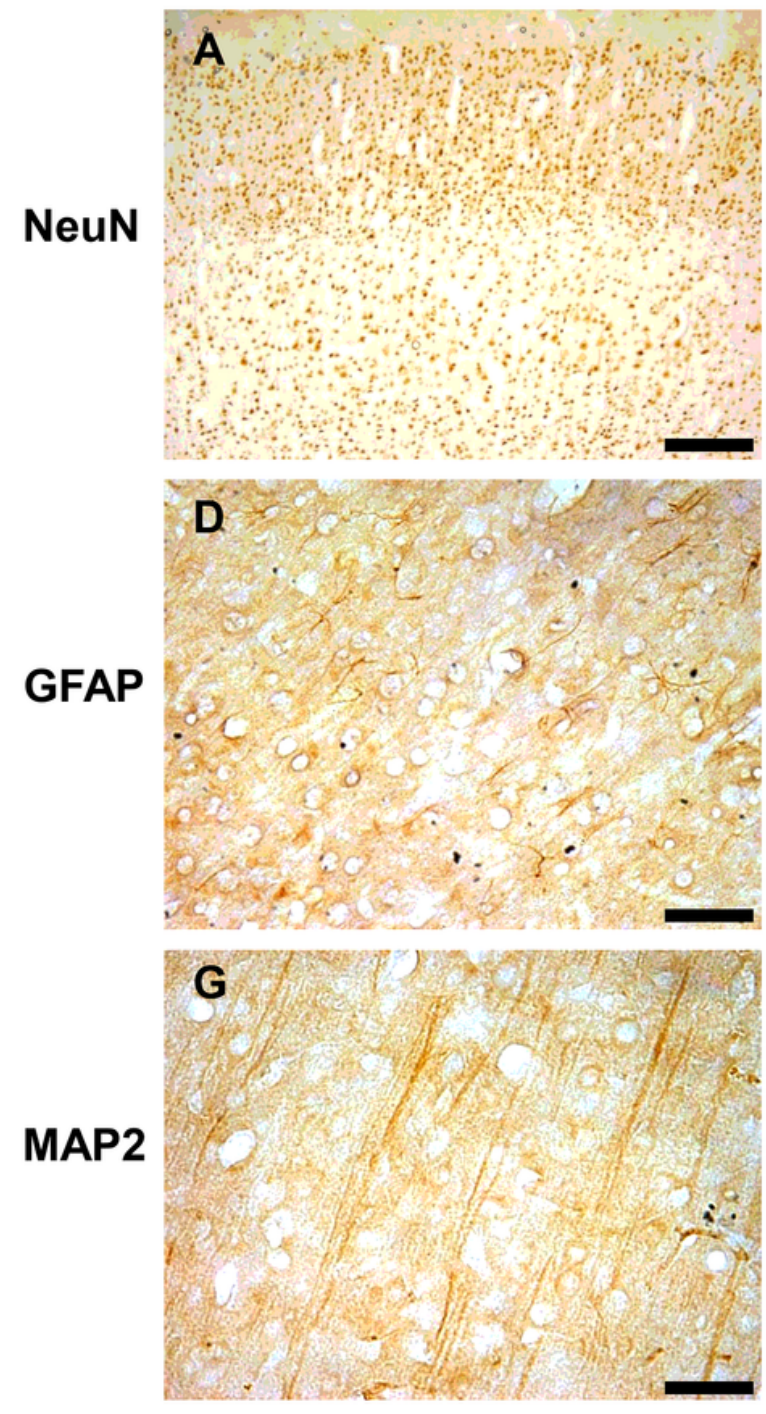

rHMGB1
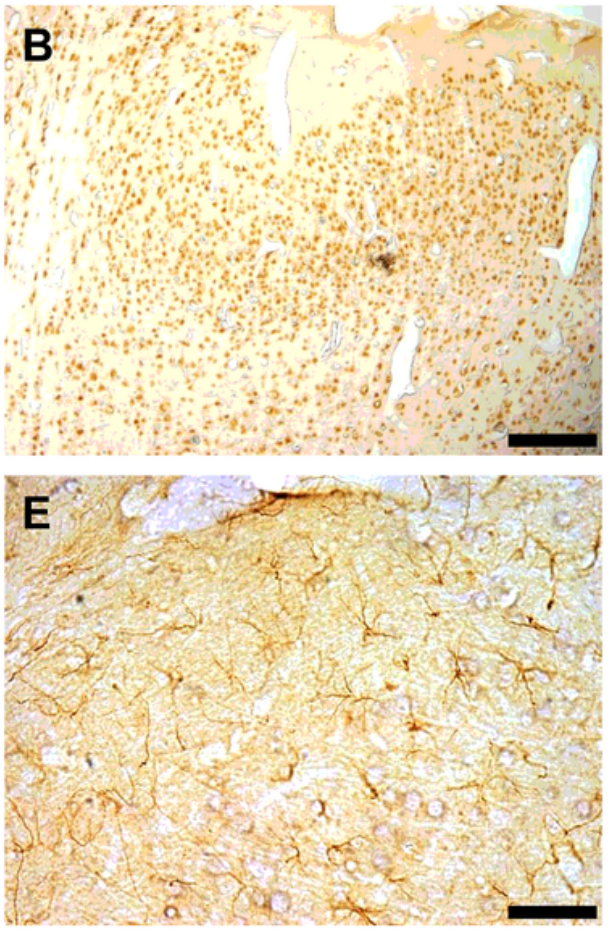

C
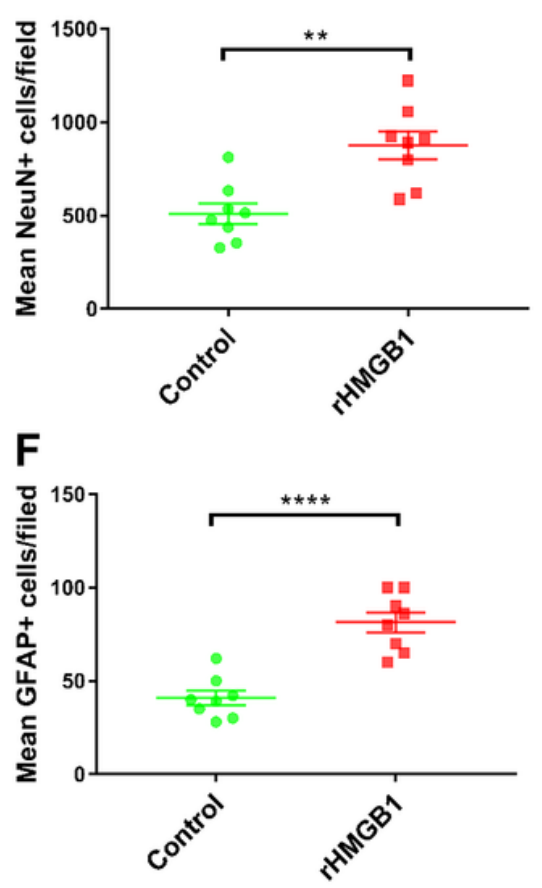

Figure 2

The immunostaining of NeuN, GFAP and MAP2 A-C: The NeuN positive cells were significantly increased in rHMGB1-treated group compared with control group. D-F: GFAP positive astrocyte were also increased in the rHMGB1-treated group compared with control group. G-H: The MAP2 staining showed the dendrites randomly oriented and crossed each other, even some dendrites were thicker than the controls. ${ }^{* *} \mathrm{p}<0.01$, $\star \star \star \star p<0.0001$. rHMGB1, rHMGB1-treated. Scale bars, $200 \mu \mathrm{m}$ for A-B; $50 \mu \mathrm{m}$ for $\mathrm{D}-\mathrm{H}$. 


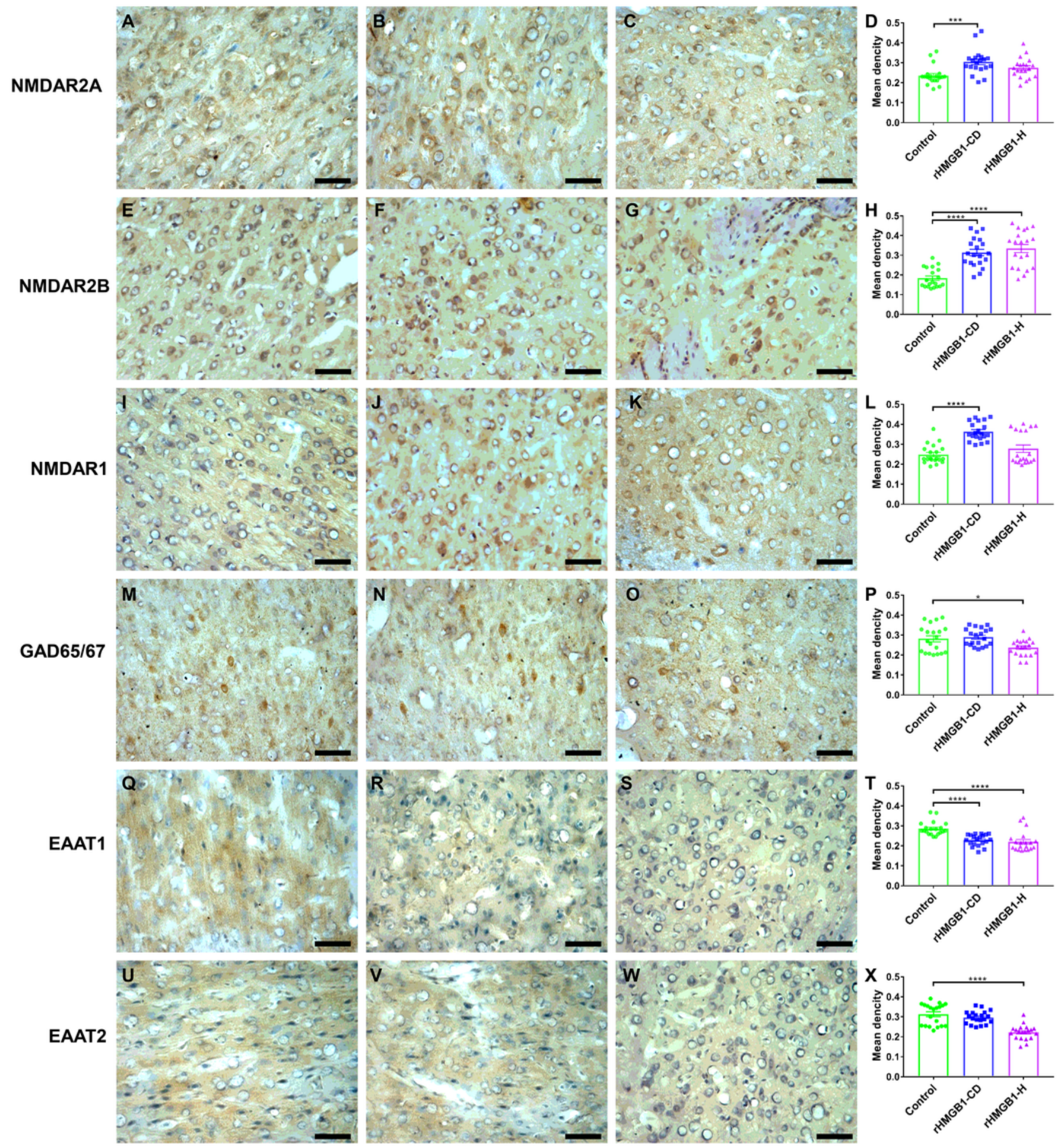

\section{Figure 3}

The immunohistochemical finding of the lesion A-H: NMDAR2 subunit immunostaining. The NR2B immunoreactivity density are significant increase in cortical lesions and heterotopia, but NR2A only increased in cortical lesions. I-L: NMDAR1 immunohistochemical staining. There was significant increase of NR1 immunoreactivity density in cortical lesions. M-P: GAD56/67 immunostaining displayed the mean immunoreactivity density was not different between control cortices and focal cortical lesions, but the 
immunoreactivity density was mild reduced in heterotopia. Q-X: EAAT immunostaining showed EAAT1 were decrease in cortical lesion and heterotopia; EAAT2 was not different between cortical lesions and control cortices; however, EAAT2 was significantly reduced in heterotopia. ${ }^{\star} p<0.05,{ }^{\star \star} p<0.01,{ }^{\star \star \star} p<0.001$, $\star * \star * p<0.0001$. rHMGB1-CD, cortical dysplasia of rHMGB1-treated rat; rHMGB1-H, heterotopia of rHMGB1treated rat. Scale bars, $50 \mu \mathrm{m}$.

Control
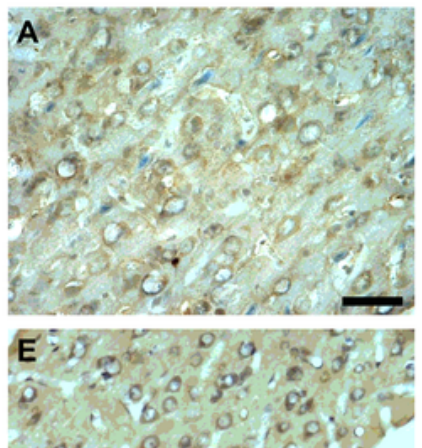

NMDAR2B
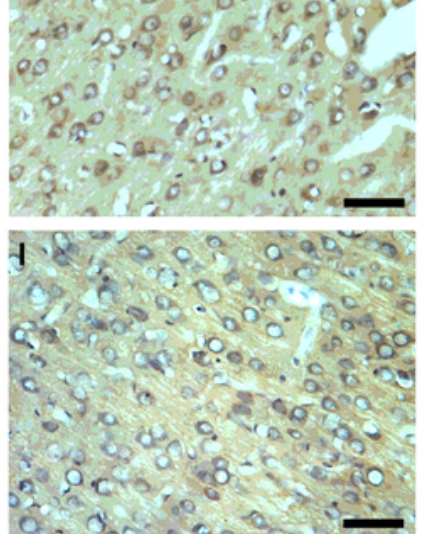

NMDAR1

GAD65/67

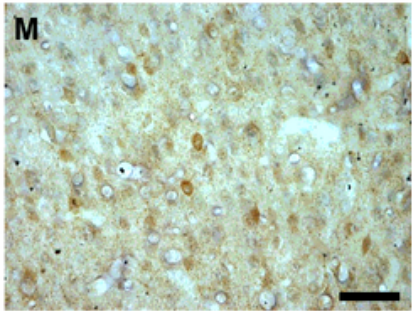

EAAT1

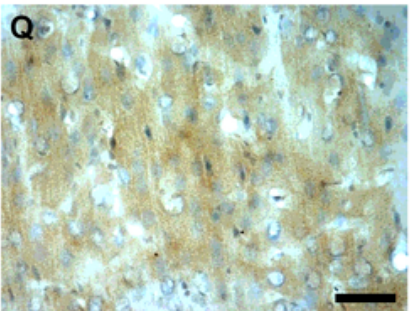

EAAT2

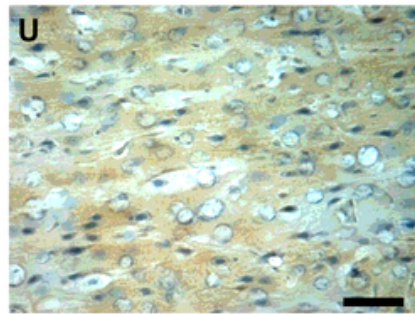

rHMGB1-CD
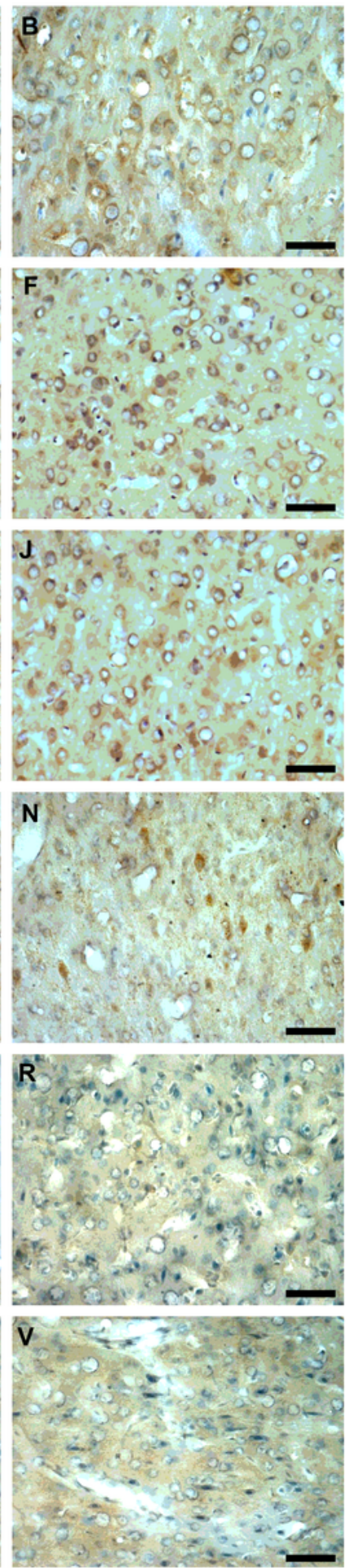

rHMGB1-H
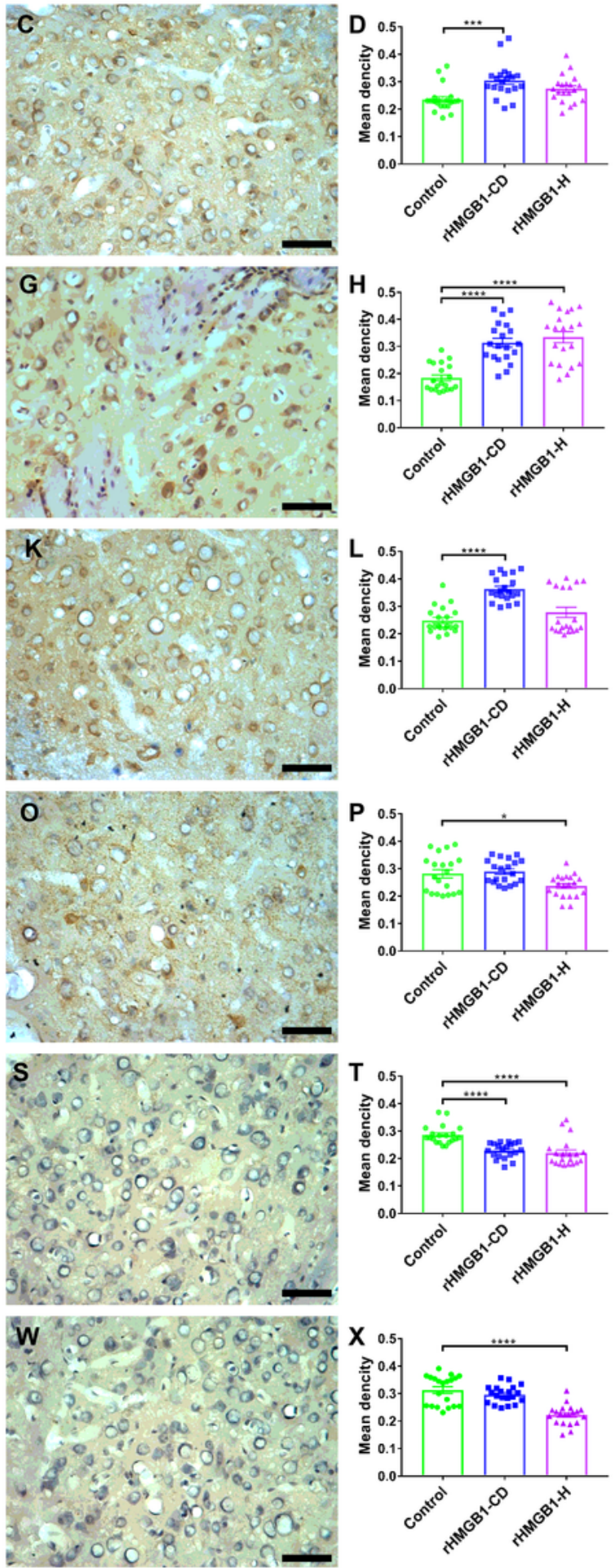

Figure 3 
The immunohistochemical finding of the lesion A-H: NMDAR2 subunit immunostaining. The NR2B immunoreactivity density are significant increase in cortical lesions and heterotopia, but NR2A only increased in cortical lesions. I-L: NMDAR1 immunohistochemical staining. There was significant increase of NR1 immunoreactivity density in cortical lesions. M-P: GAD56/67 immunostaining displayed the mean immunoreactivity density was not different between control cortices and focal cortical lesions, but the immunoreactivity density was mild reduced in heterotopia. Q-X: EAAT immunostaining showed EAAT1 were decrease in cortical lesion and heterotopia; EAAT2 was not different between cortical lesions and control cortices; however, EAAT2 was significantly reduced in heterotopia. ${ }^{*} p<0.05,{ }^{\star \star} p<0.01,{ }^{\star \star *} p<0.001$, $\star \star \star \star * p<0.0001$. rHMGB1-CD, cortical dysplasia of rHMGB1-treated rat; rHMGB1-H, heterotopia of rHMGB1treated rat. Scale bars, $50 \mu \mathrm{m}$. 
Control

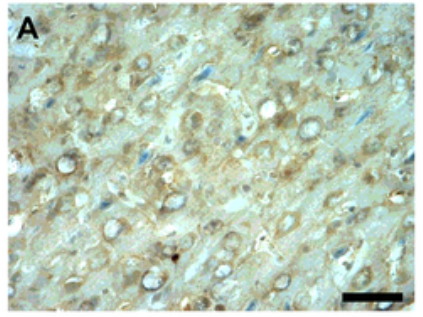

NMDAR2A

NMDAR2B

NMDAR1

GAD65/67
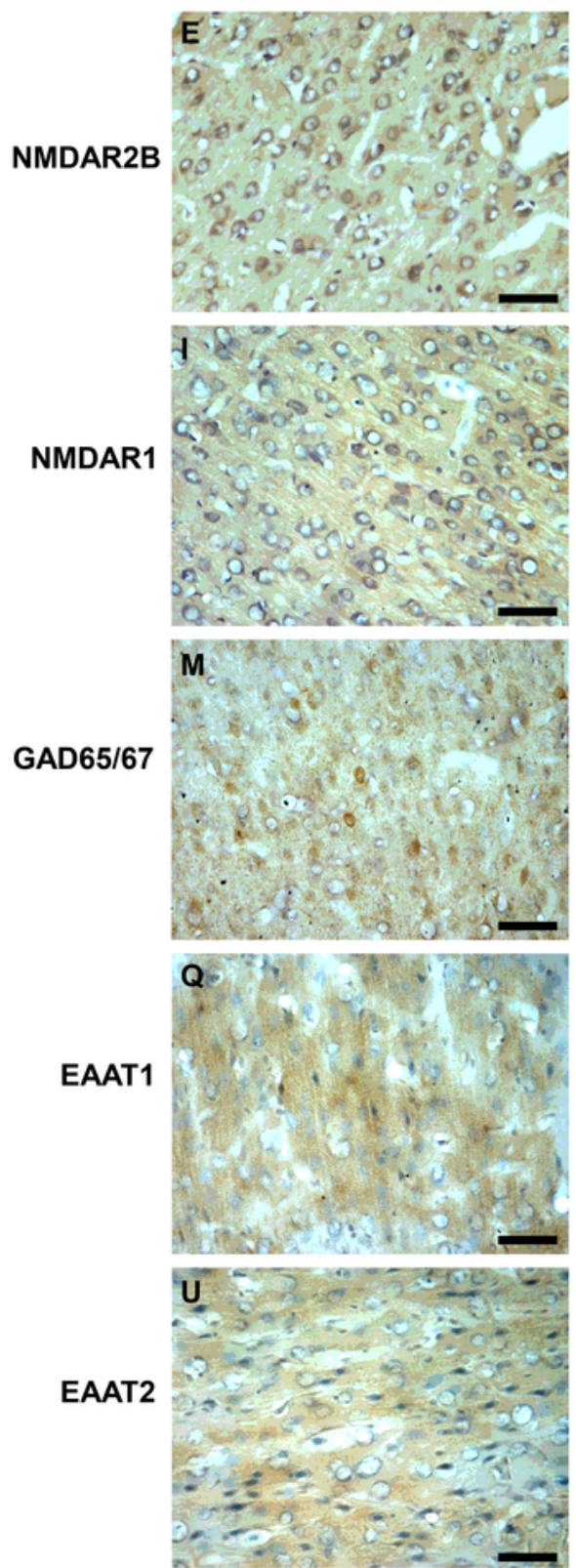

rHMGB1-CD
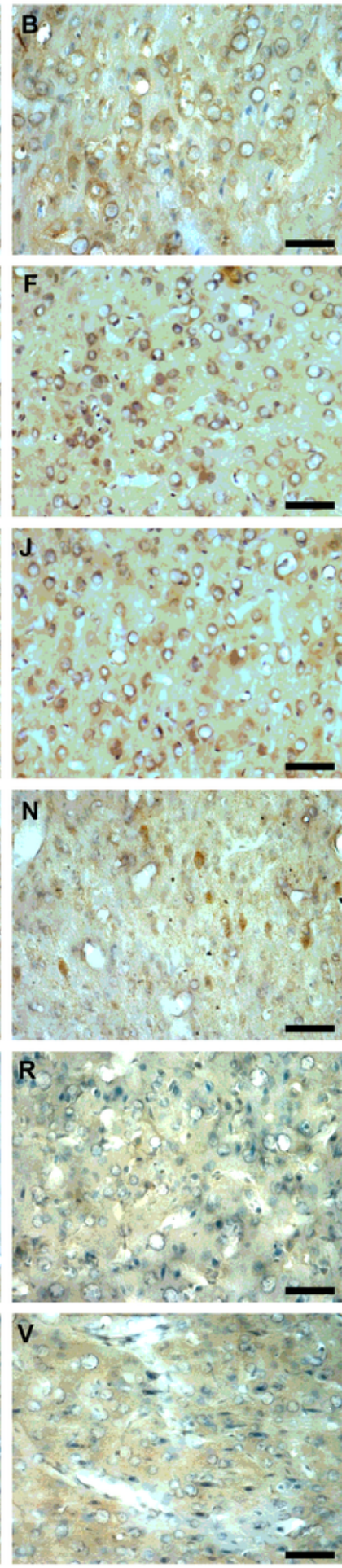

rHMGB1-H
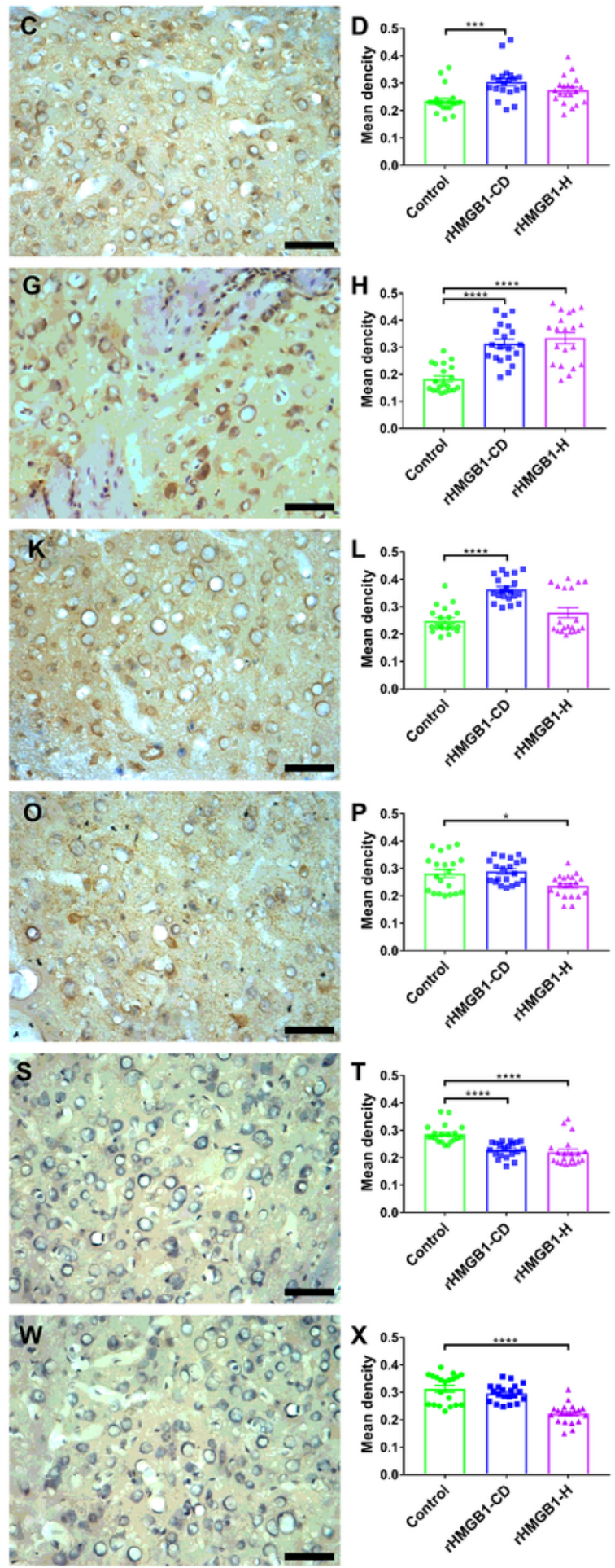

\section{Figure 3}

The immunohistochemical finding of the lesion A-H: NMDAR2 subunit immunostaining. The NR2B immunoreactivity density are significant increase in cortical lesions and heterotopia, but NR2A only increased in cortical lesions. I-L: NMDAR1 immunohistochemical staining. There was significant increase of NR1 immunoreactivity density in cortical lesions. M-P: GAD56/67 immunostaining displayed the mean immunoreactivity density was not different between control cortices and focal cortical lesions, but the 
immunoreactivity density was mild reduced in heterotopia. Q-X: EAAT immunostaining showed EAAT1 were decrease in cortical lesion and heterotopia; EAAT2 was not different between cortical lesions and control cortices; however, EAAT2 was significantly reduced in heterotopia. ${ }^{\star} p<0.05,{ }^{\star \star} p<0.01,{ }^{\star \star \star} p<0.001$, $\star * \star * p<0.0001$. rHMGB1-CD, cortical dysplasia of rHMGB1-treated rat; rHMGB1-H, heterotopia of rHMGB1treated rat. Scale bars, $50 \mu \mathrm{m}$.

Control
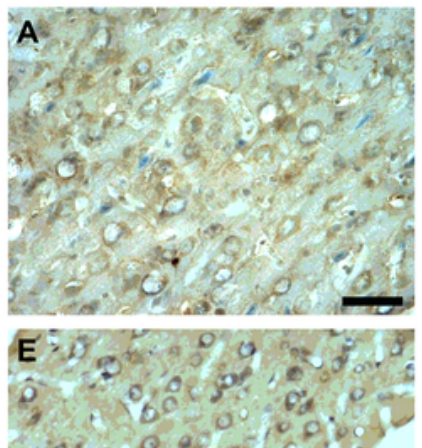

NMDAR2B
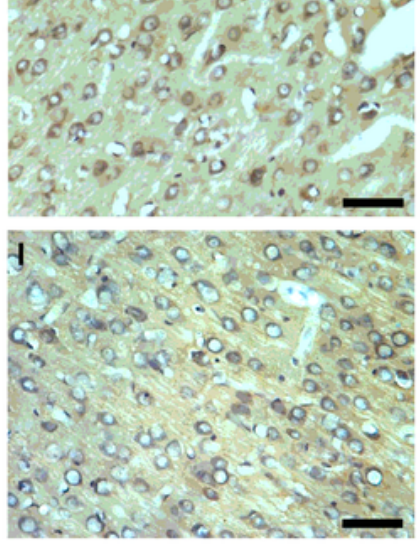

NMDAR1

GAD65/67

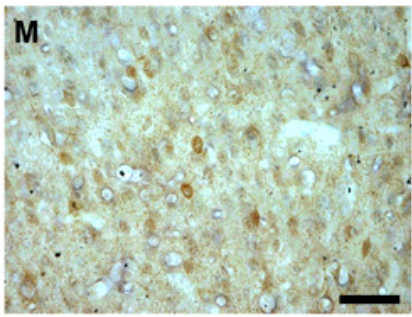

EAAT1

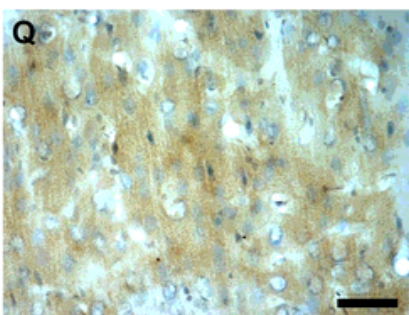

EAAT2

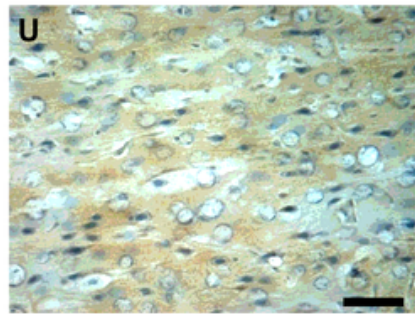

rHMGB1-CD
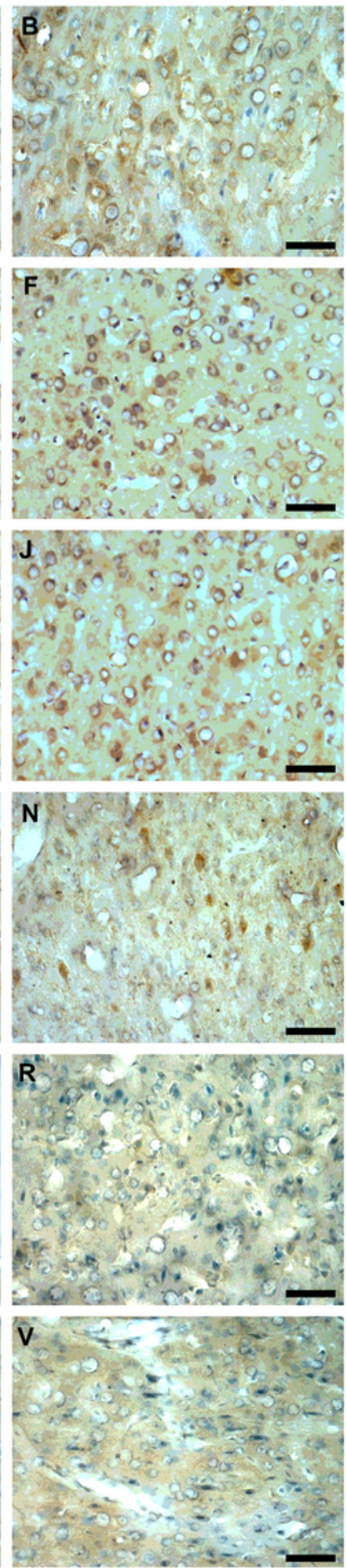

rHMGB1-H
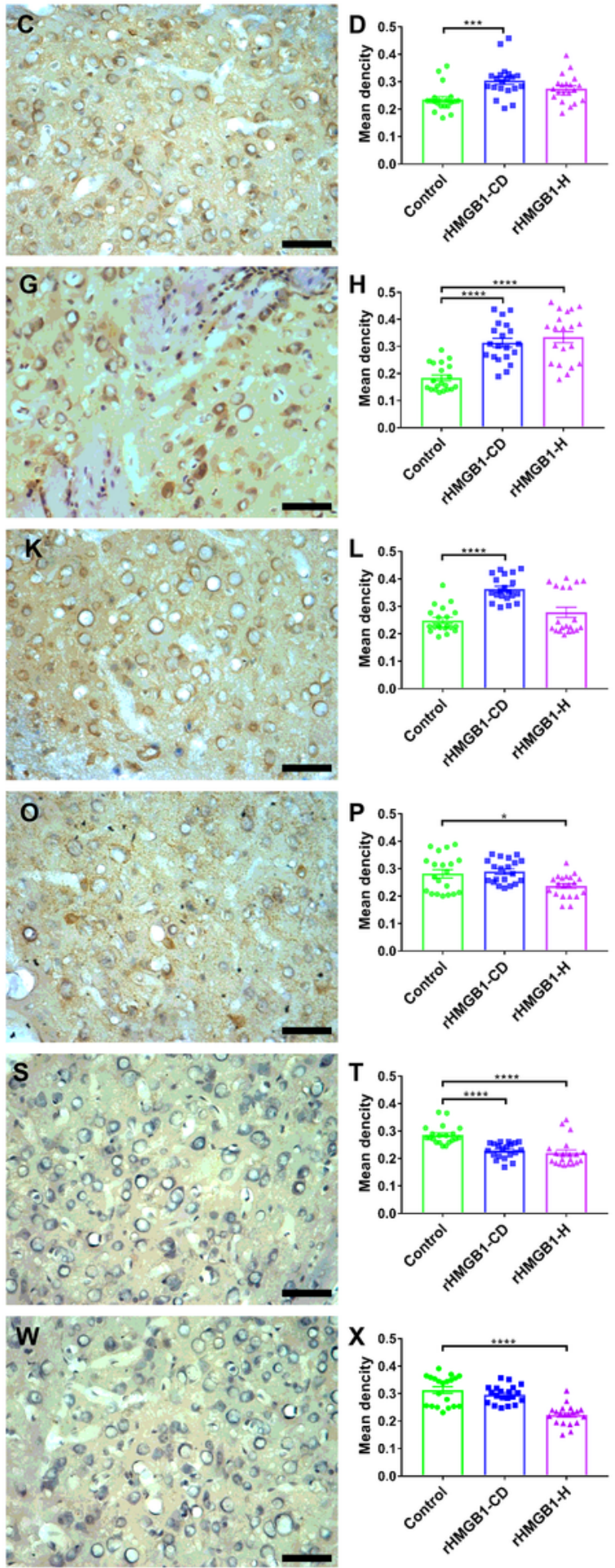

Figure 3 
The immunohistochemical finding of the lesion A-H: NMDAR2 subunit immunostaining. The NR2B immunoreactivity density are significant increase in cortical lesions and heterotopia, but NR2A only increased in cortical lesions. I-L: NMDAR1 immunohistochemical staining. There was significant increase of NR1 immunoreactivity density in cortical lesions. M-P: GAD56/67 immunostaining displayed the mean immunoreactivity density was not different between control cortices and focal cortical lesions, but the immunoreactivity density was mild reduced in heterotopia. Q-X: EAAT immunostaining showed EAAT1 were decrease in cortical lesion and heterotopia; EAAT2 was not different between cortical lesions and control cortices; however, EAAT2 was significantly reduced in heterotopia. ${ }^{*} p<0.05,{ }^{* \star} p<0.01,{ }^{* \star \star} p<0.001$, $\star \star \star \star * p<0.0001$. rHMGB1-CD, cortical dysplasia of rHMGB1-treated rat; rHMGB1-H, heterotopia of rHMGB1treated rat. Scale bars, $50 \mu \mathrm{m}$.

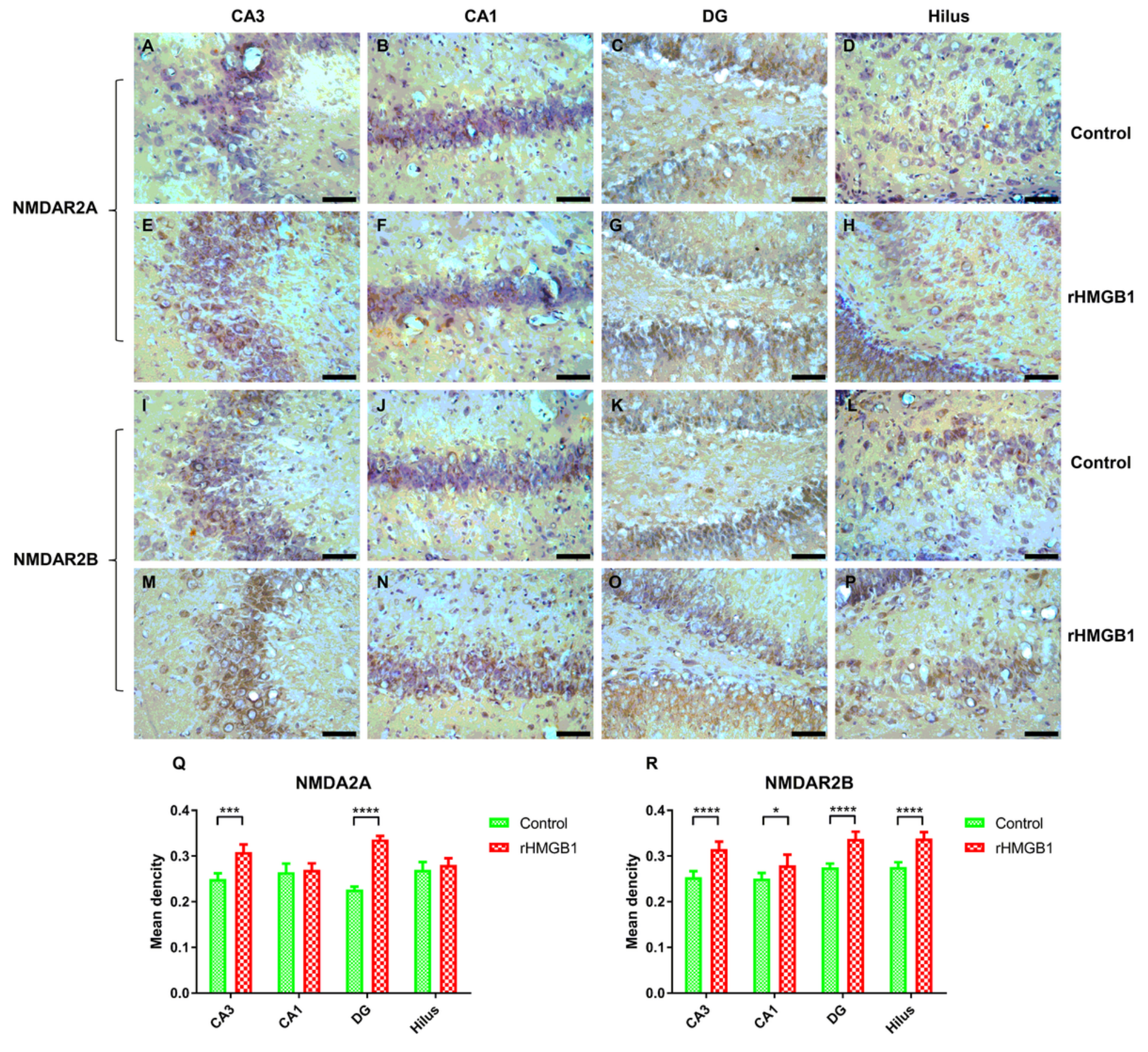

Figure 4 
The immunohistochemical examination of the hippocampus A-H, Q: The results showed there were stronger immunoreactivity of NR2A in CA3 and DG of rHMGB1-treated rats than controls; but there was no difference in CA1 and Hilus. I-P, R: Immunoreactivity of NR2B were stronger in CA3, CA1, DG and Hilus of rHMGB1-treated group than control group. ${ }^{*} p<0.05,{ }^{* \star *} p<0.001,{ }^{* \star * *} p<0.0001$. rHMGB1, rHMGB1treated. Scale bars, $50 \mu \mathrm{m}$.

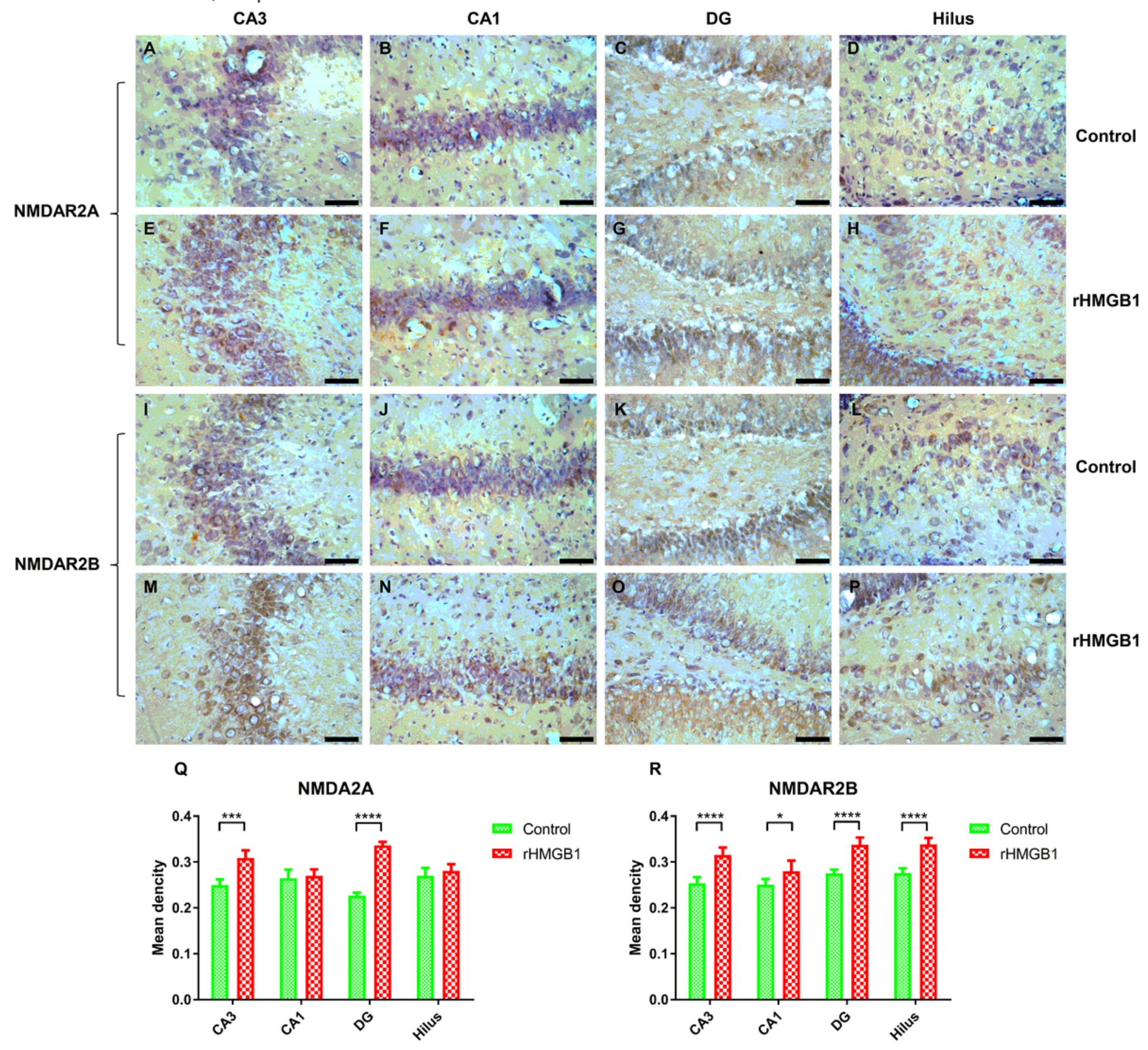

Figure 4

The immunohistochemical examination of the hippocampus $\mathrm{A}-\mathrm{H}, \mathrm{Q}$ : The results showed there were stronger immunoreactivity of NR2A in CA3 and DG of rHMGB1-treated rats than controls; but there was no difference in CA1 and Hilus. I-P, R: Immunoreactivity of NR2B were stronger in CA3, CA1, DG and Hilus 
of rHMGB1-treated group than control group. ${ }^{\star} p<0.05,{ }^{* \star *} \mathrm{p}<0.001,{ }^{\star * \star *} \mathrm{p}<0.0001$. rHMGB1, rHMGB1treated. Scale bars, $50 \mu \mathrm{m}$.

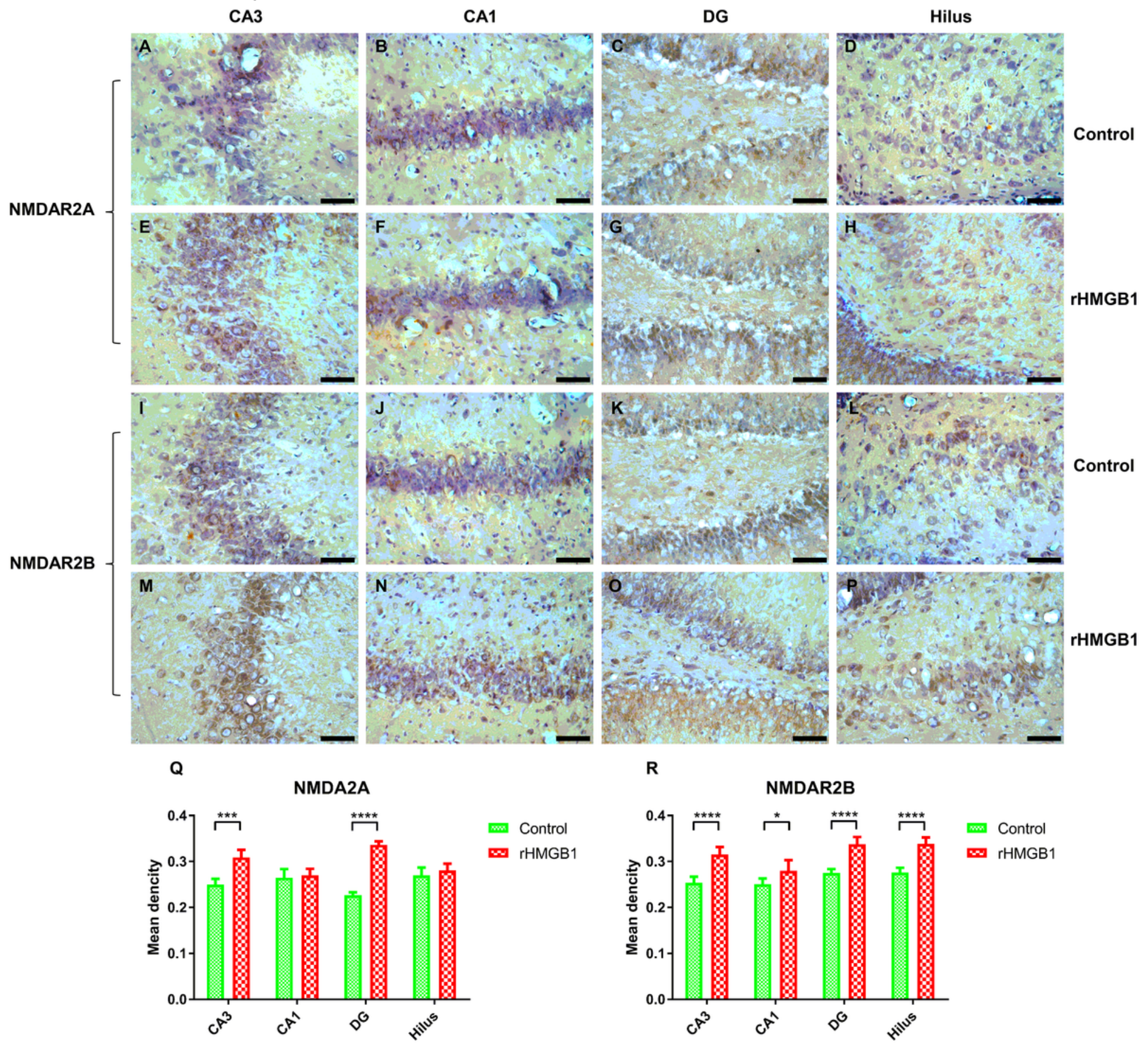

Figure 4

The immunohistochemical examination of the hippocampus $\mathrm{A}-\mathrm{H}, \mathrm{Q}$ : The results showed there were stronger immunoreactivity of NR2A in CA3 and DG of rHMGB1-treated rats than controls; but there was no difference in CA1 and Hilus. I-P, R: Immunoreactivity of NR2B were stronger in CA3, CA1, DG and Hilus of rHMGB1-treated group than control group. ${ }^{*} p<0.05,{ }^{\star \star *} p<0.001,{ }^{\star \star \star *} p<0.0001$. rHMGB1, rHMGB1treated. Scale bars, $50 \mu \mathrm{m}$. 


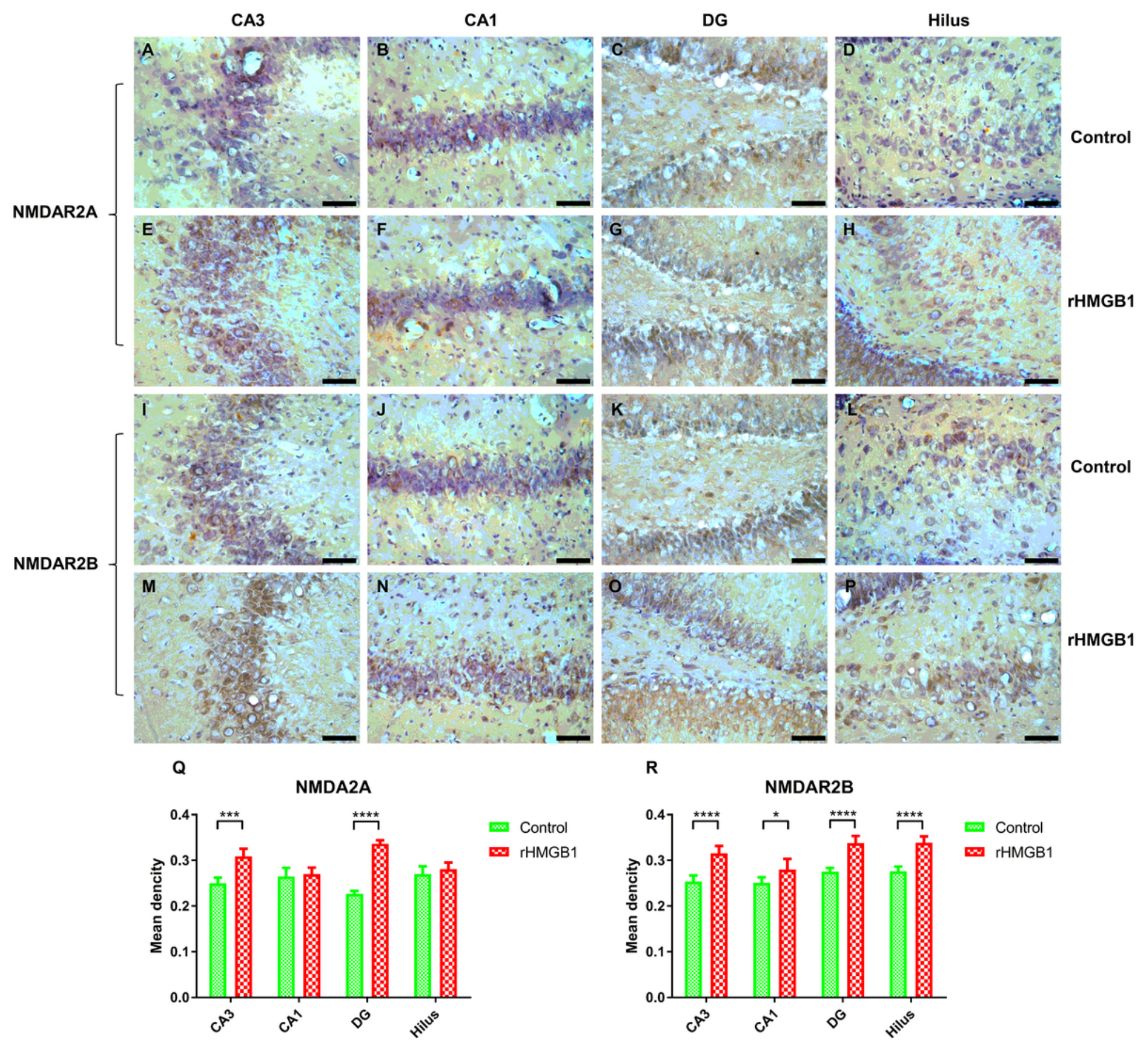

Figure 4

The immunohistochemical examination of the hippocampus A-H, Q: The results showed there were stronger immunoreactivity of NR2A in CA3 and DG of rHMGB1-treated rats than controls; but there was no difference in CA1 and Hilus. I-P, R: Immunoreactivity of NR2B were stronger in CA3, CA1, DG and Hilus of rHMGB1-treated group than control group. ${ }^{*} p<0.05,{ }^{\star \star \star} \mathrm{p}<0.001,{ }^{\star \star \star \star *} p<0.0001$. rHMGB1, rHMGB1treated. Scale bars, $50 \mu \mathrm{m}$. 
A

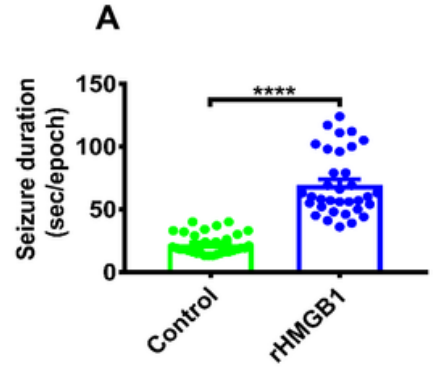

E
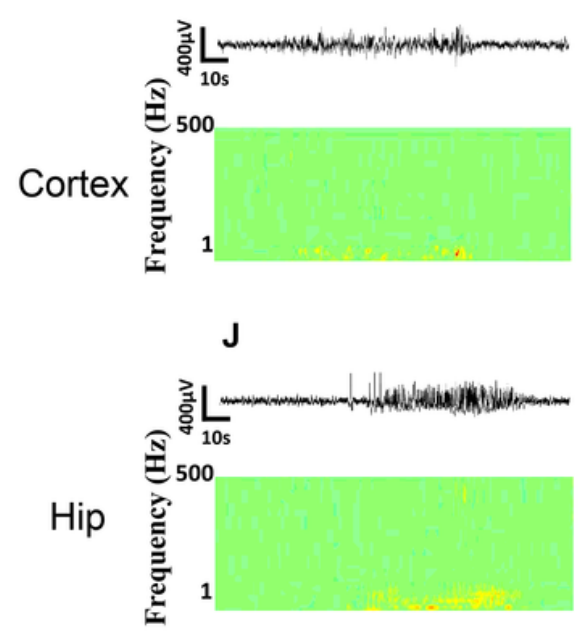

B
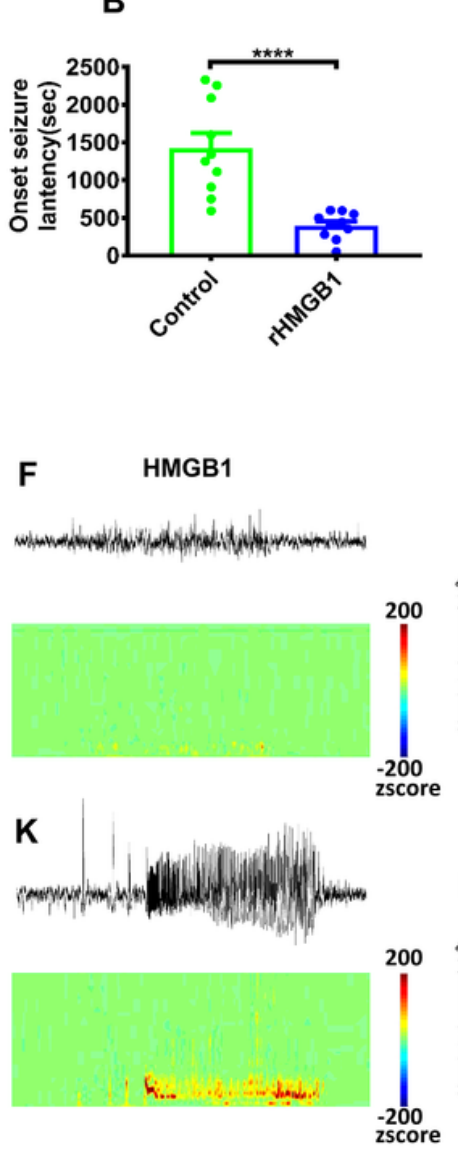

C
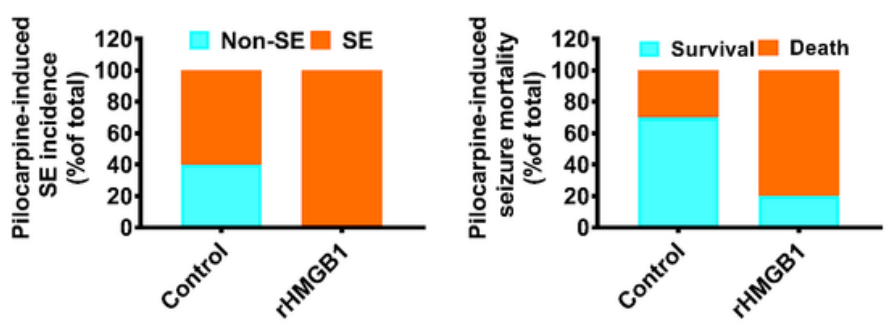

G
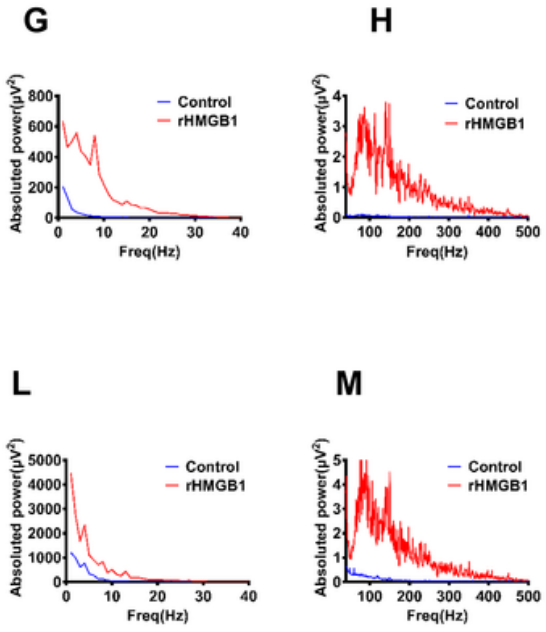

M

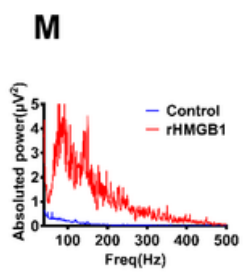

$\mathbf{N}$

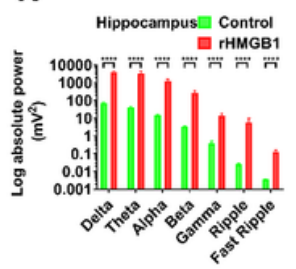

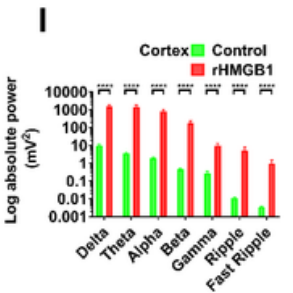

\section{Figure 5}

Pilocarpine-induced effects were obtained from rHMGB1-treated rats and controls A-B: The onset latency of seizures was shorter in rHMGB1-treated rats after pilocarpine administered intracerebroventricularly, and the duration of seizure were longer than controls. C-D: The rate of status epilepsy and mortality were higher in rHMGB1-treated group than controls. E-N: The raw EEG showed the amplitude were significantly increase; The time-frequency analysis displayed all bands power were significantly higher in rHMGB1treated rats than controls during seizure after pilocarpine injection. Especially, the around $4 \mathrm{~Hz}$ and $150 \mathrm{~Hz}$ bands power is the most significant in both cortex and hippocampus, but only the around $8 \mathrm{~Hz}$ peak in cortex. ${ }^{* \star \star \star} p<0.0001, \mathrm{SE}$, status epilepsy; rHMGB1, rHMGB1-treated. Hip, hippocampus. 
A

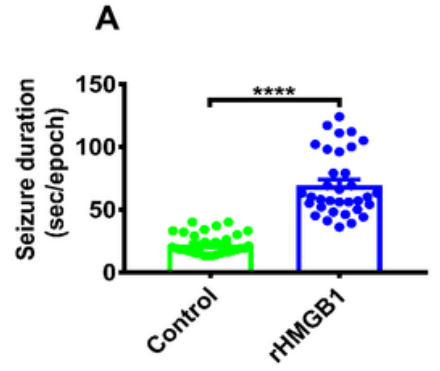

$E$
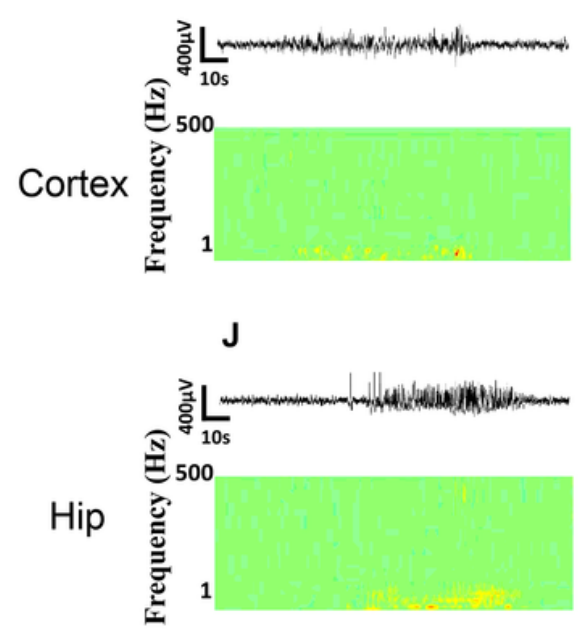

B
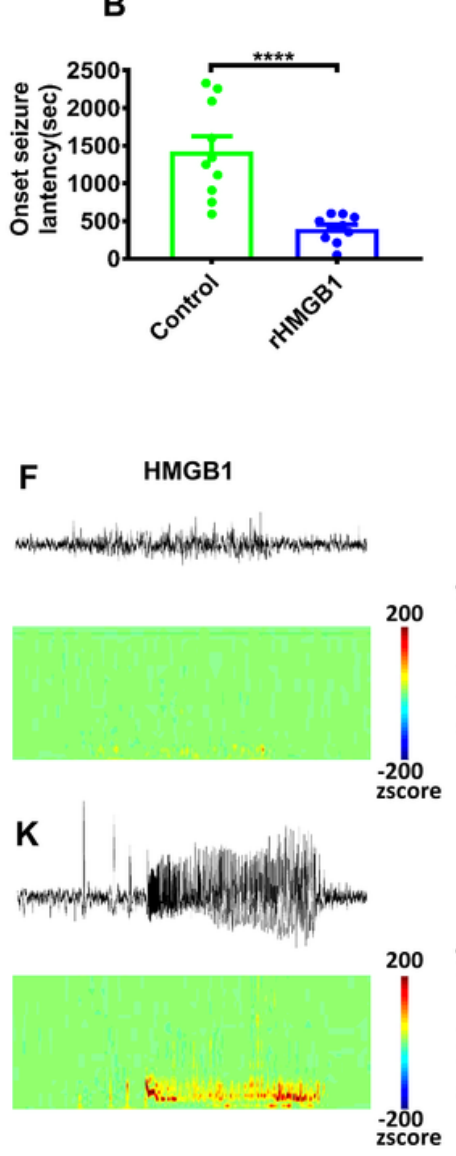

C
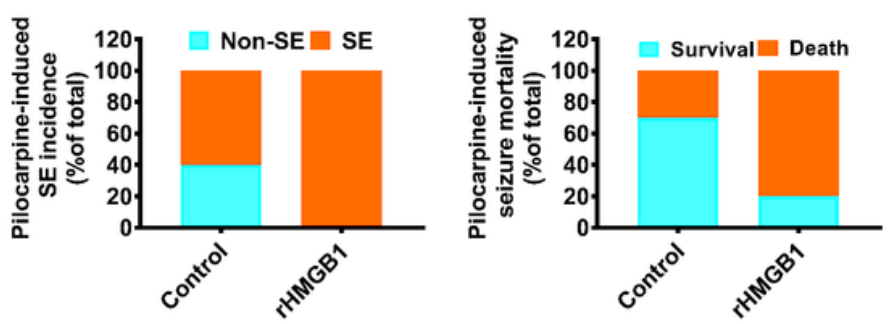

G
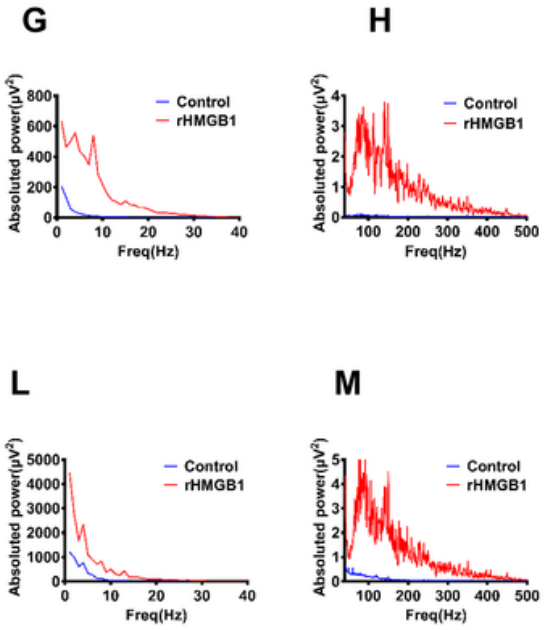

M

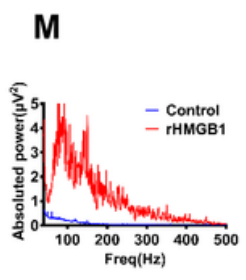

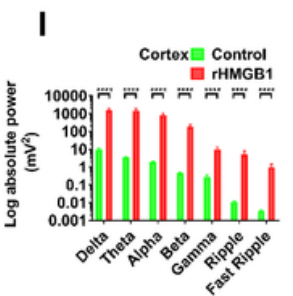

$\mathbf{N}$

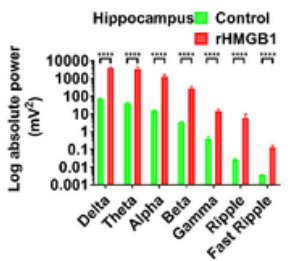

\section{Figure 5}

Pilocarpine-induced effects were obtained from rHMGB1-treated rats and controls A-B: The onset latency of seizures was shorter in rHMGB1-treated rats after pilocarpine administered intracerebroventricularly, and the duration of seizure were longer than controls. C-D: The rate of status epilepsy and mortality were higher in rHMGB1-treated group than controls. E-N: The raw EEG showed the amplitude were significantly increase; The time-frequency analysis displayed all bands power were significantly higher in rHMGB1treated rats than controls during seizure after pilocarpine injection. Especially, the around $4 \mathrm{~Hz}$ and $150 \mathrm{~Hz}$ bands power is the most significant in both cortex and hippocampus, but only the around $8 \mathrm{~Hz}$ peak in cortex. ${ }^{* \star \star \star} p<0.0001, \mathrm{SE}$, status epilepsy; rHMGB1, rHMGB1-treated. Hip, hippocampus. 
A

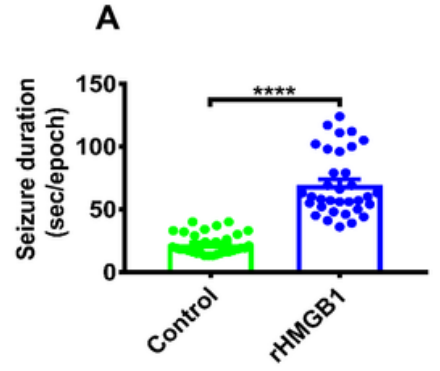

E
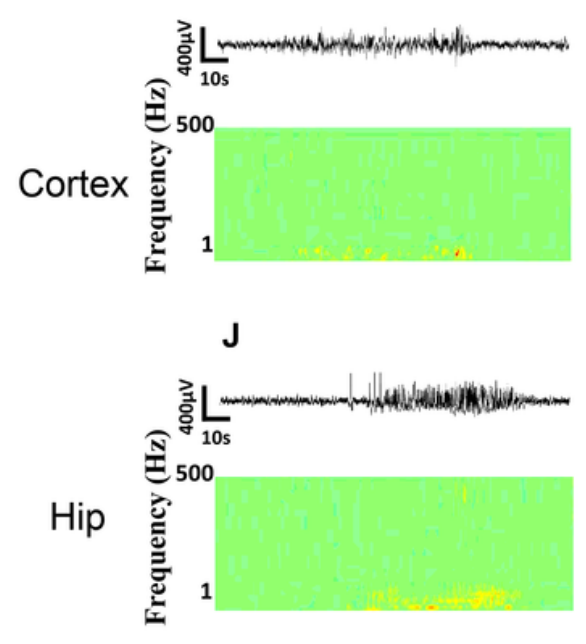

B
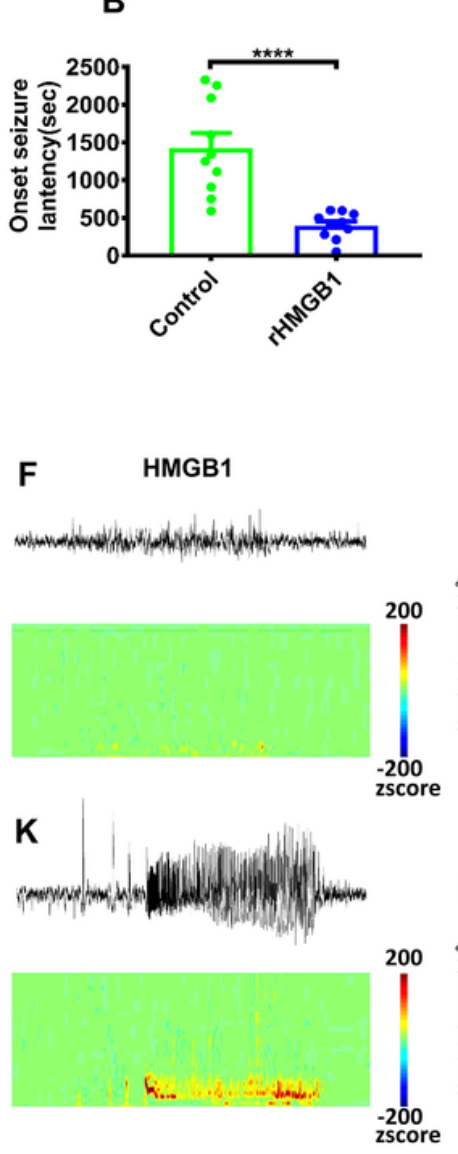

C
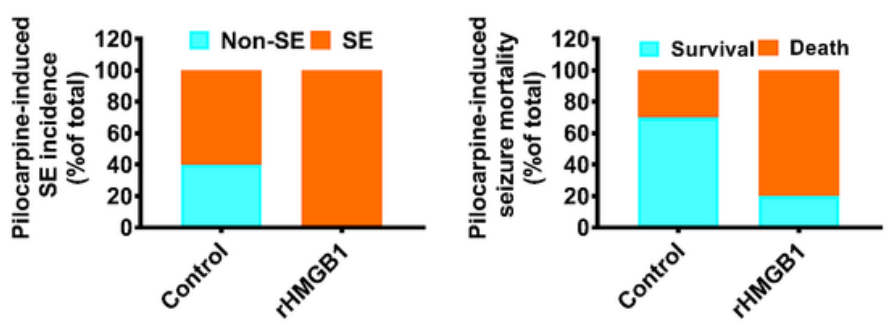

G
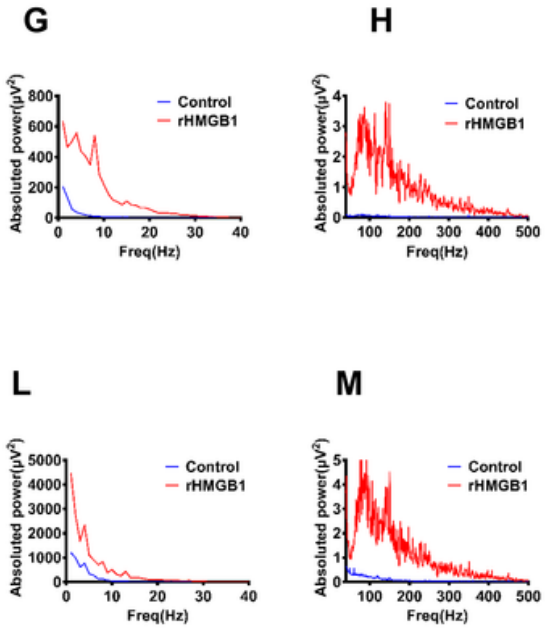

M

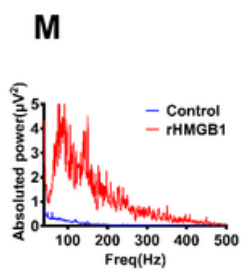

$\mathbf{N}$

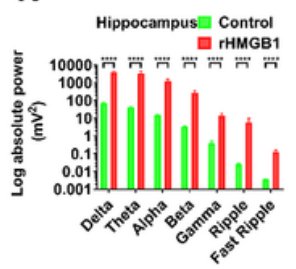

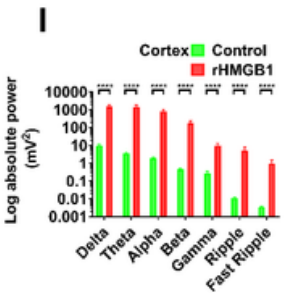

\section{Figure 5}

Pilocarpine-induced effects were obtained from rHMGB1-treated rats and controls A-B: The onset latency of seizures was shorter in rHMGB1-treated rats after pilocarpine administered intracerebroventricularly, and the duration of seizure were longer than controls. C-D: The rate of status epilepsy and mortality were higher in rHMGB1-treated group than controls. E-N: The raw EEG showed the amplitude were significantly increase; The time-frequency analysis displayed all bands power were significantly higher in rHMGB1treated rats than controls during seizure after pilocarpine injection. Especially, the around $4 \mathrm{~Hz}$ and $150 \mathrm{~Hz}$ bands power is the most significant in both cortex and hippocampus, but only the around $8 \mathrm{~Hz}$ peak in cortex. ${ }^{* \star \star \star} p<0.0001, \mathrm{SE}$, status epilepsy; rHMGB1, rHMGB1-treated. Hip, hippocampus. 
A

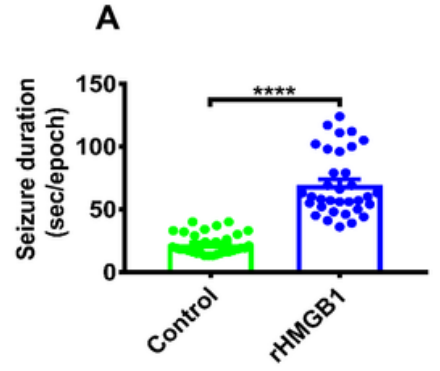

E
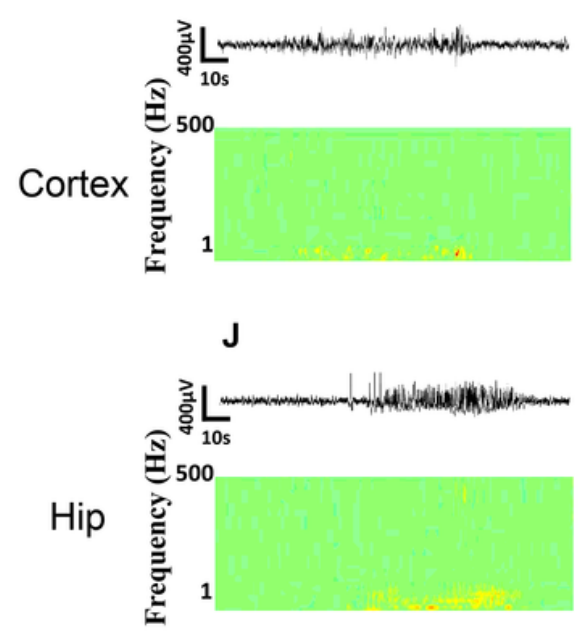

B
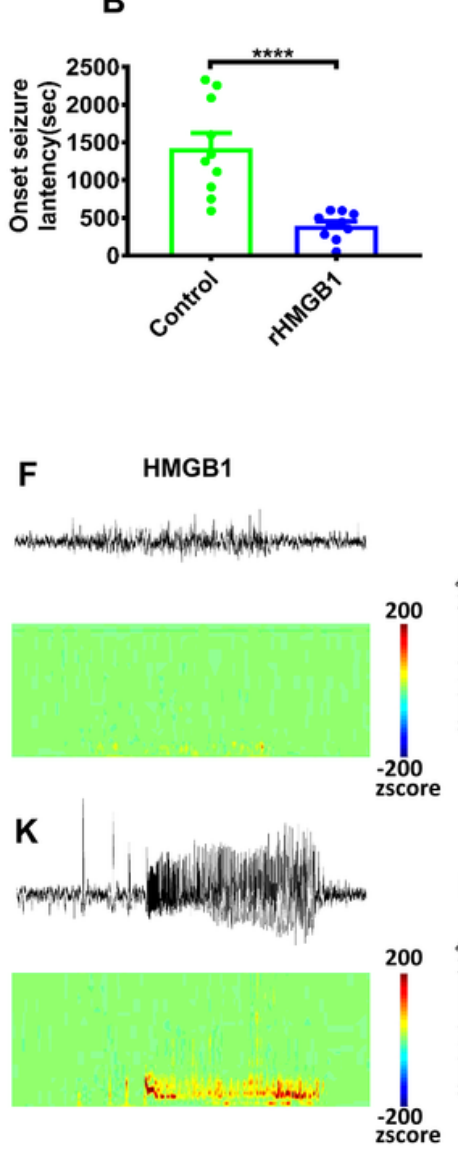

C
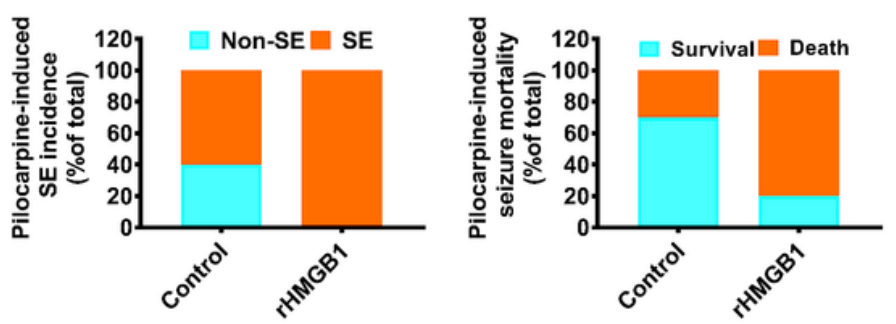

G
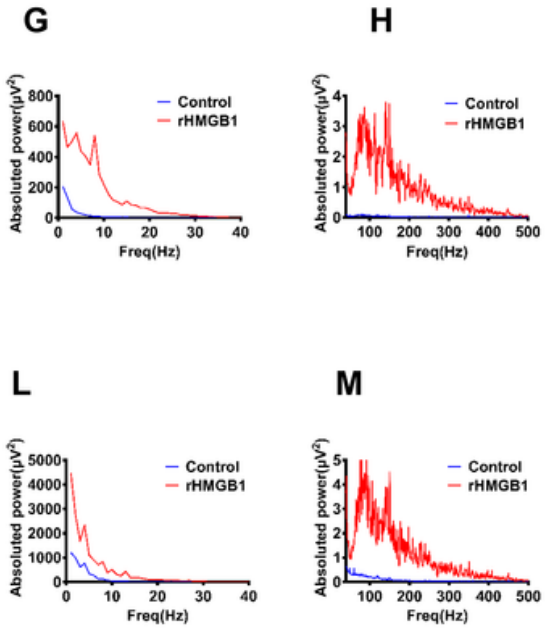

M

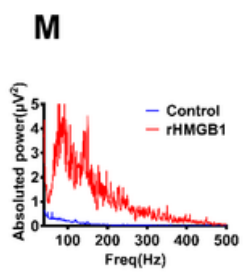

$\mathbf{N}$

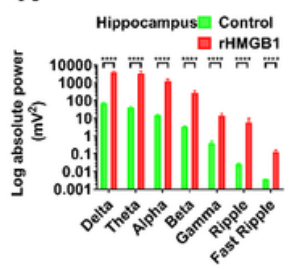

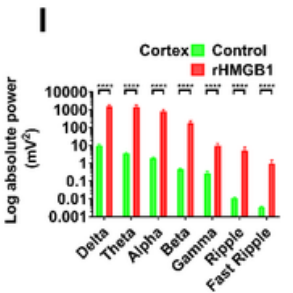

\section{Figure 5}

Pilocarpine-induced effects were obtained from rHMGB1-treated rats and controls A-B: The onset latency of seizures was shorter in rHMGB1-treated rats after pilocarpine administered intracerebroventricularly, and the duration of seizure were longer than controls. C-D: The rate of status epilepsy and mortality were higher in rHMGB1-treated group than controls. E-N: The raw EEG showed the amplitude were significantly increase; The time-frequency analysis displayed all bands power were significantly higher in rHMGB1treated rats than controls during seizure after pilocarpine injection. Especially, the around $4 \mathrm{~Hz}$ and $150 \mathrm{~Hz}$ bands power is the most significant in both cortex and hippocampus, but only the around $8 \mathrm{~Hz}$ peak in cortex. ${ }^{* \star \star \star} p<0.0001, \mathrm{SE}$, status epilepsy; rHMGB1, rHMGB1-treated. Hip, hippocampus. 

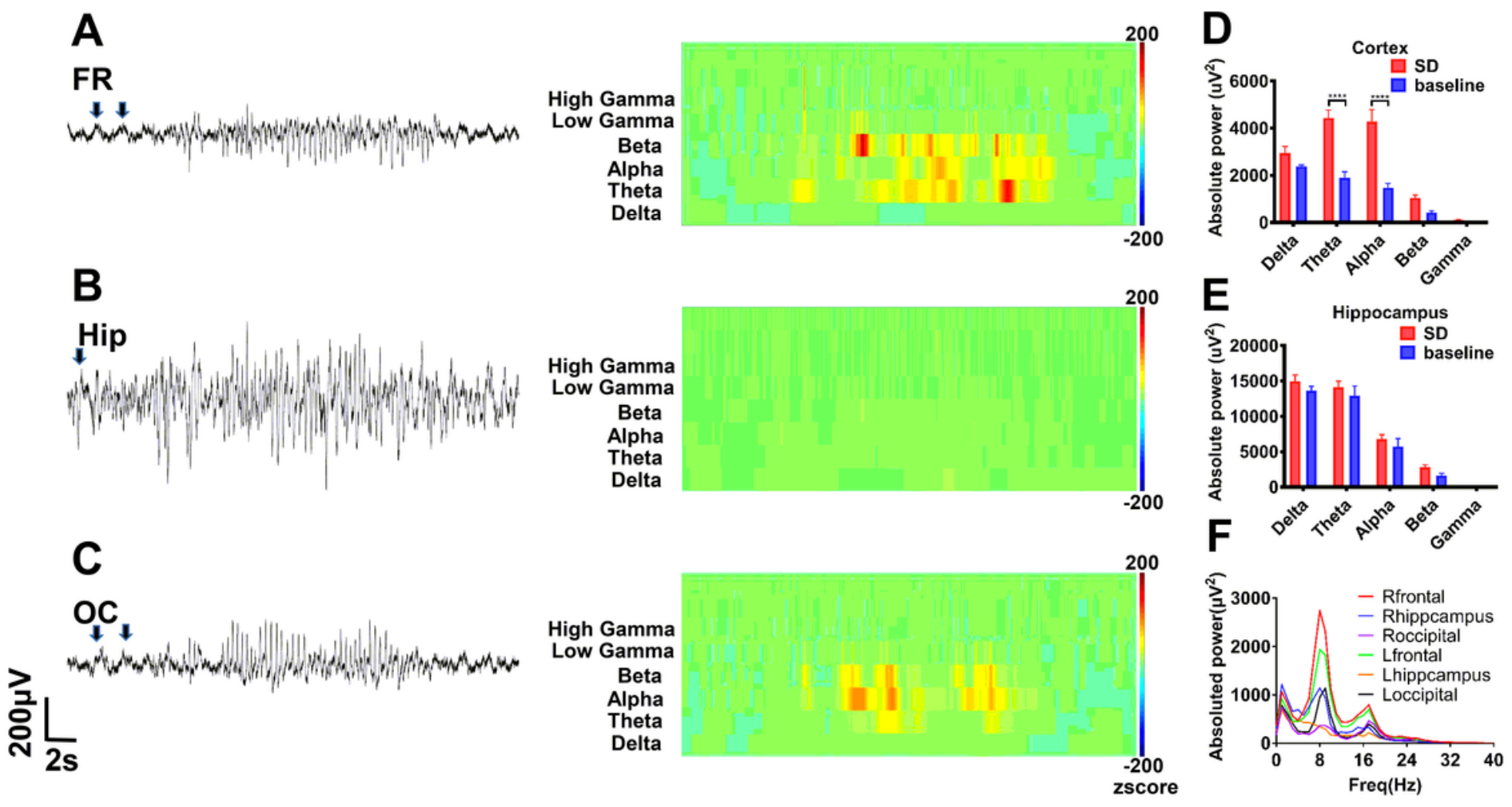

Figure 6

The rHMGB1-treated rats displayed non-convulsive spontaneous spike-wave discharges A-C: The raw EEG recording and wavelet analysis of nonconvulsive spontaneous discharges; the theta, alpha, beta and gamma power were increased in rHMGB1-treated group and the slow wave was before spontaneous spike-wave discharges(arrows). D-E: The statistics of different bands power demonstrated compare with self-baseline, only the cortical powers of theta and alpha bands were significantly increased. $F$ : The power spectra showed around $8 \mathrm{~Hz}$ is the most significant on frontal electrode. ${ }^{\star \star \star \star} \mathrm{p}<0.0001$. FR, frontal lobe; Hip, hippocampus; OC, occipital lobe; SD, spontaneous discharges. 


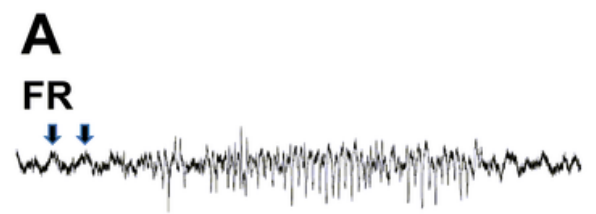

B
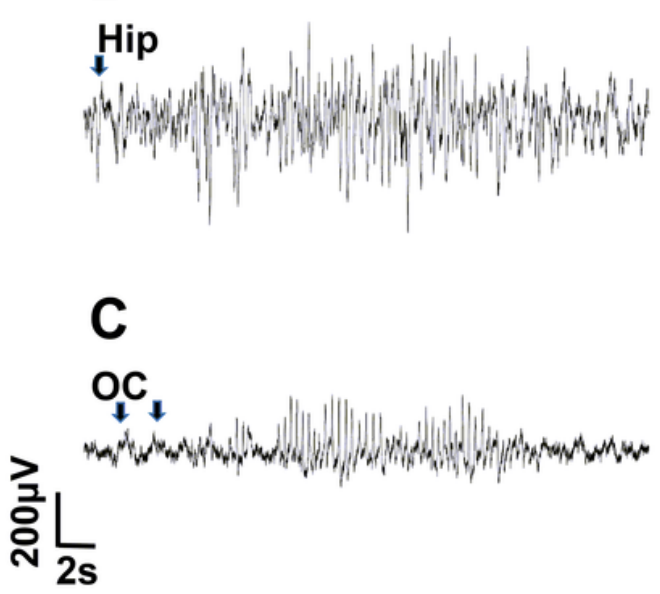
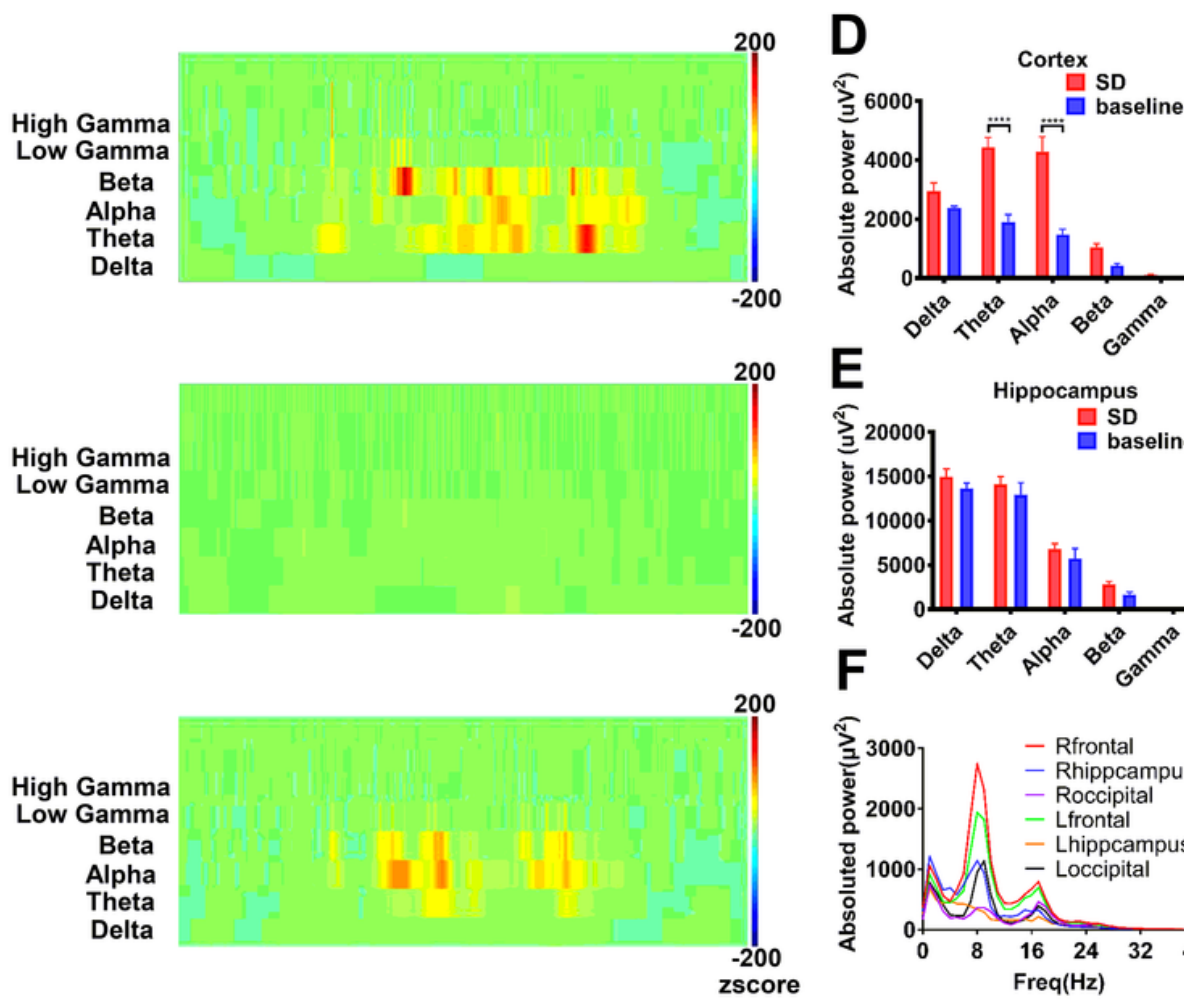

\section{Figure 6}

The rHMGB1-treated rats displayed non-convulsive spontaneous spike-wave discharges A-C: The raw EEG recording and wavelet analysis of nonconvulsive spontaneous discharges; the theta, alpha, beta and gamma power were increased in rHMGB1-treated group and the slow wave was before spontaneous spike-wave discharges(arrows). D-E: The statistics of different bands power demonstrated compare with self-baseline, only the cortical powers of theta and alpha bands were significantly increased. $F$ : The power spectra showed around $8 \mathrm{~Hz}$ is the most significant on frontal electrode. ${ }^{\star \star \star \star} \mathrm{p}<0.0001$. FR, frontal lobe; Hip, hippocampus; OC, occipital lobe; SD, spontaneous discharges. 


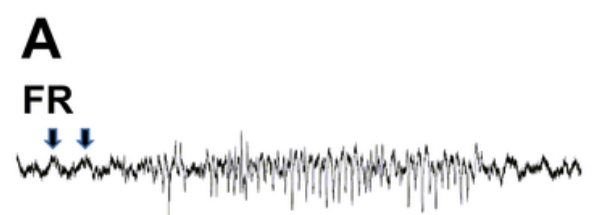

B
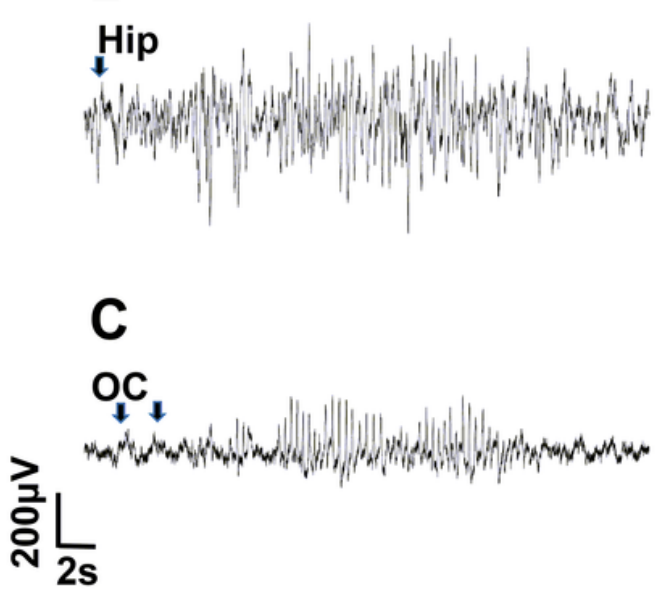
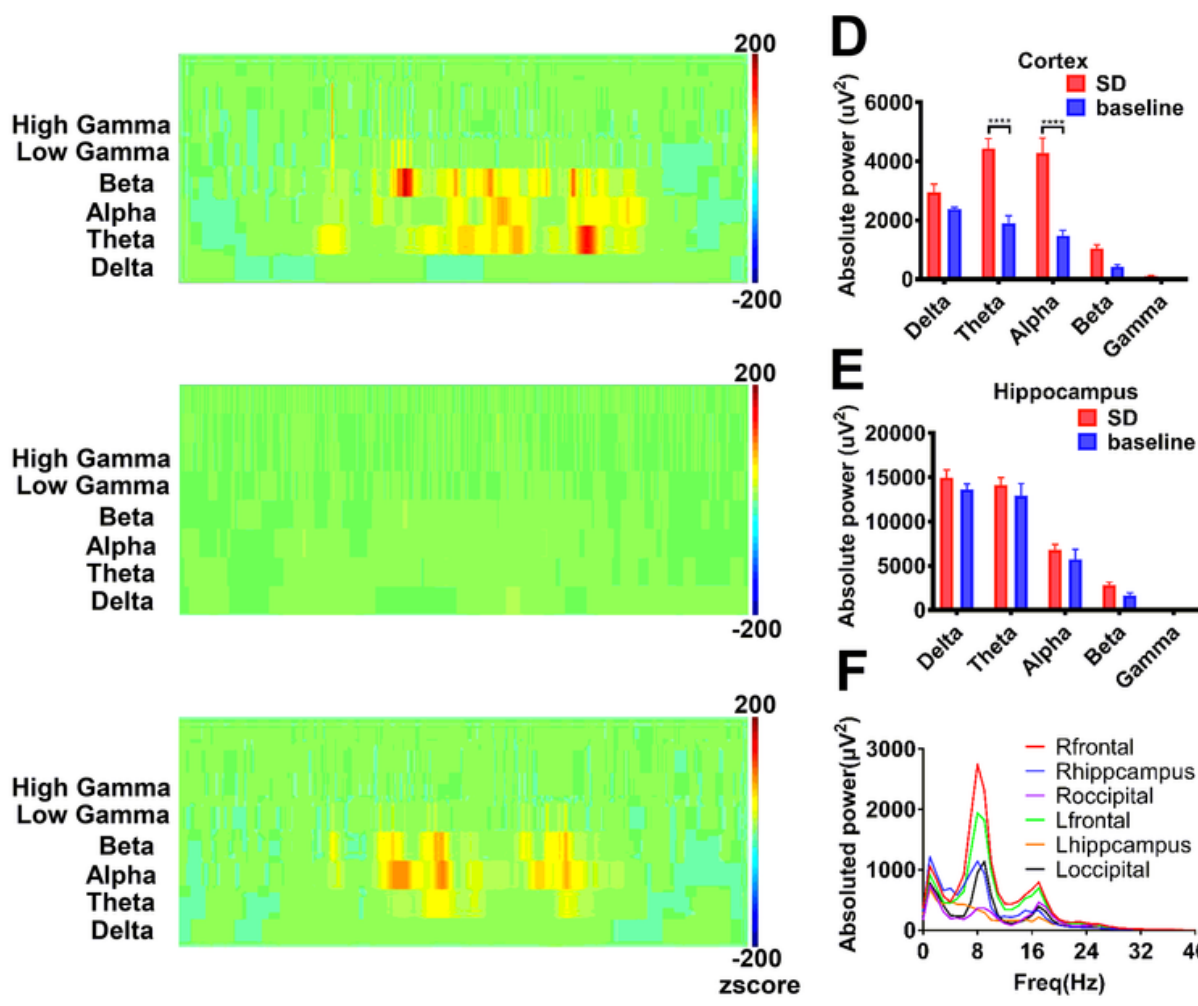

\section{Figure 6}

The rHMGB1-treated rats displayed non-convulsive spontaneous spike-wave discharges A-C: The raw EEG recording and wavelet analysis of nonconvulsive spontaneous discharges; the theta, alpha, beta and gamma power were increased in rHMGB1-treated group and the slow wave was before spontaneous spike-wave discharges(arrows). D-E: The statistics of different bands power demonstrated compare with self-baseline, only the cortical powers of theta and alpha bands were significantly increased. $F$ : The power spectra showed around $8 \mathrm{~Hz}$ is the most significant on frontal electrode. ${ }^{\star \star \star \star} \mathrm{p}<0.0001$. FR, frontal lobe; Hip, hippocampus; OC, occipital lobe; SD, spontaneous discharges. 


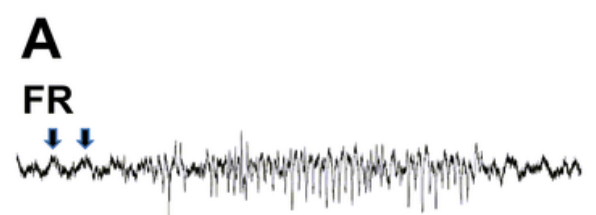

B
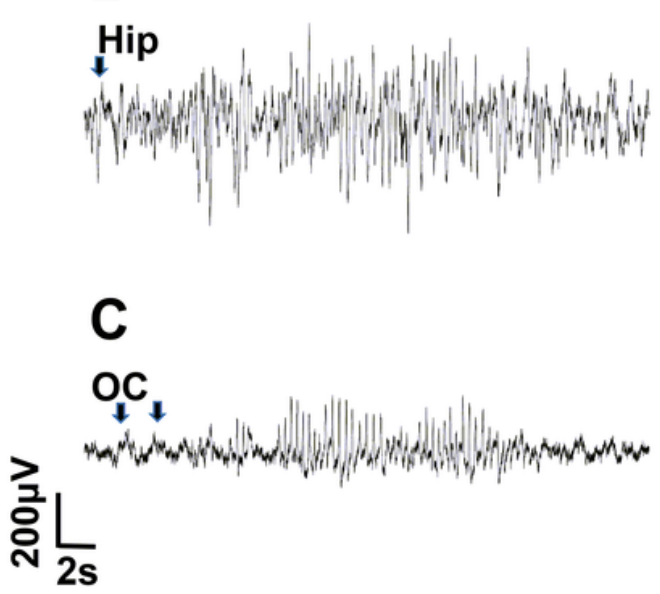
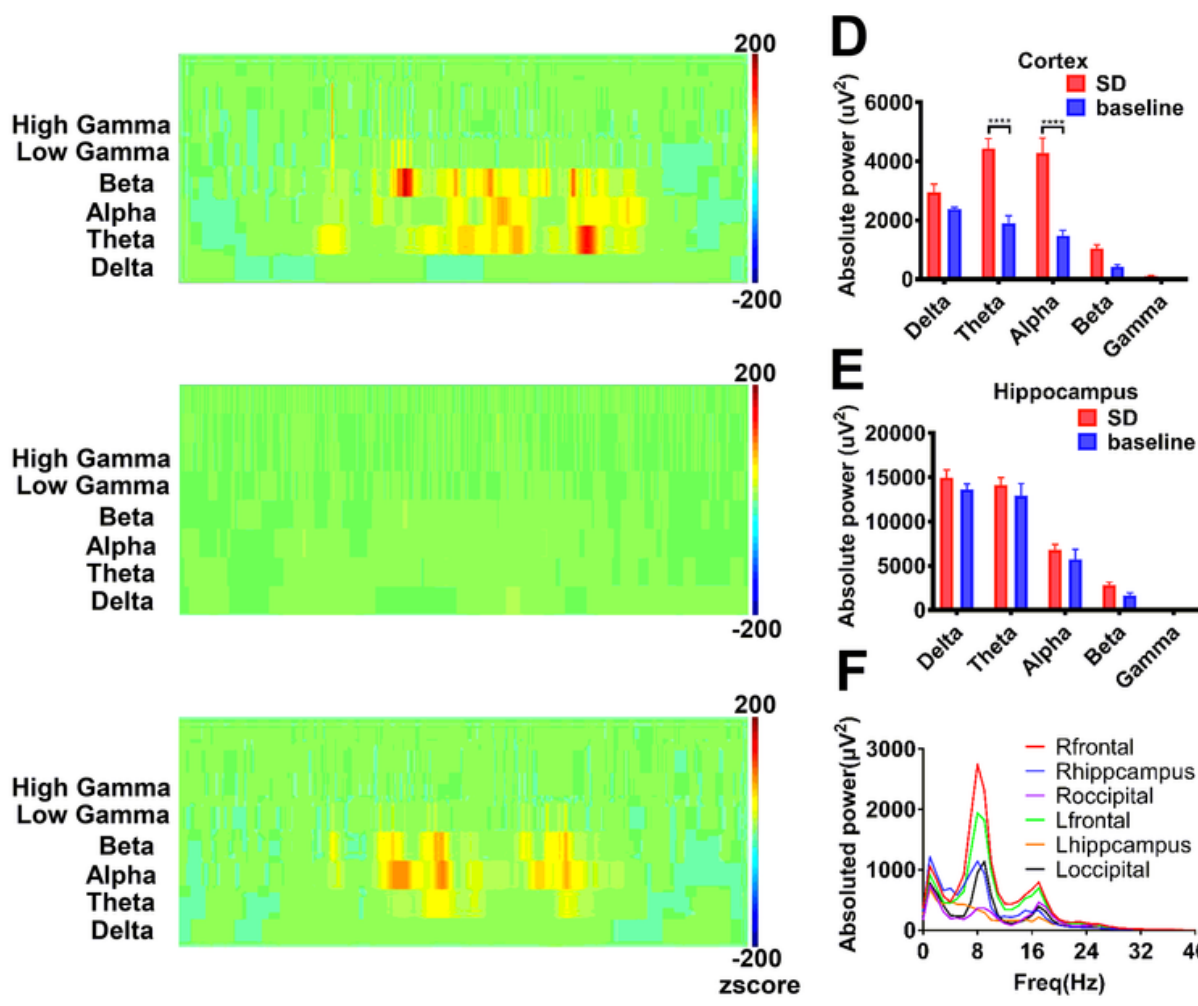

\section{Figure 6}

The rHMGB1-treated rats displayed non-convulsive spontaneous spike-wave discharges A-C: The raw EEG recording and wavelet analysis of nonconvulsive spontaneous discharges; the theta, alpha, beta and gamma power were increased in rHMGB1-treated group and the slow wave was before spontaneous spike-wave discharges(arrows). D-E: The statistics of different bands power demonstrated compare with self-baseline, only the cortical powers of theta and alpha bands were significantly increased. $F$ : The power spectra showed around $8 \mathrm{~Hz}$ is the most significant on frontal electrode. ${ }^{\star \star \star \star} \mathrm{p}<0.0001$. FR, frontal lobe; Hip, hippocampus; OC, occipital lobe; SD, spontaneous discharges. 


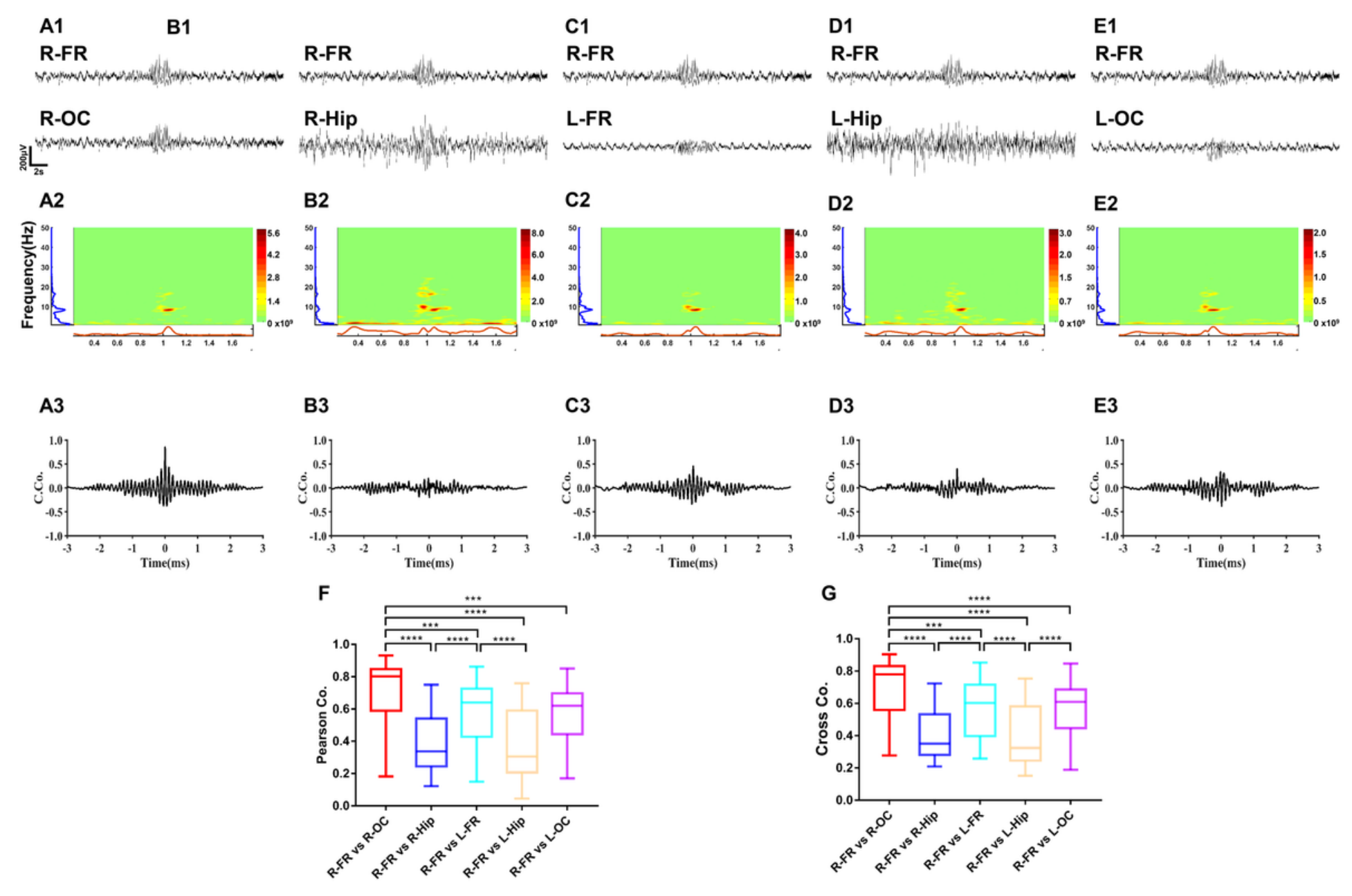

\section{Figure 7}

The synchrony degree of spontaneous discharges between different brain regions. The results showed the right frontal cortex and occipital cortex had the highest degree of synchrony(A1-G). Intriguing, synchronization of spontaneous spike-wave discharges was concentrated to $8 \mathrm{~Hz}$ (A2-E2). The coherence and cross-correlation analysis also showed right frontal cortex and occipital cortex had the maximum coefficient, secondly the coefficient between right frontal cortex and left frontal cortex(A3-G). Nonetheless, the spontaneous spike-wave discharges between cortex and hippocampus displayed various degree of

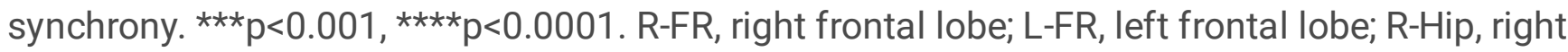
hippocampus; L-Hip, left hippocampus; R-OC, right occipital lobe; L-OC, left occipital lobe. 


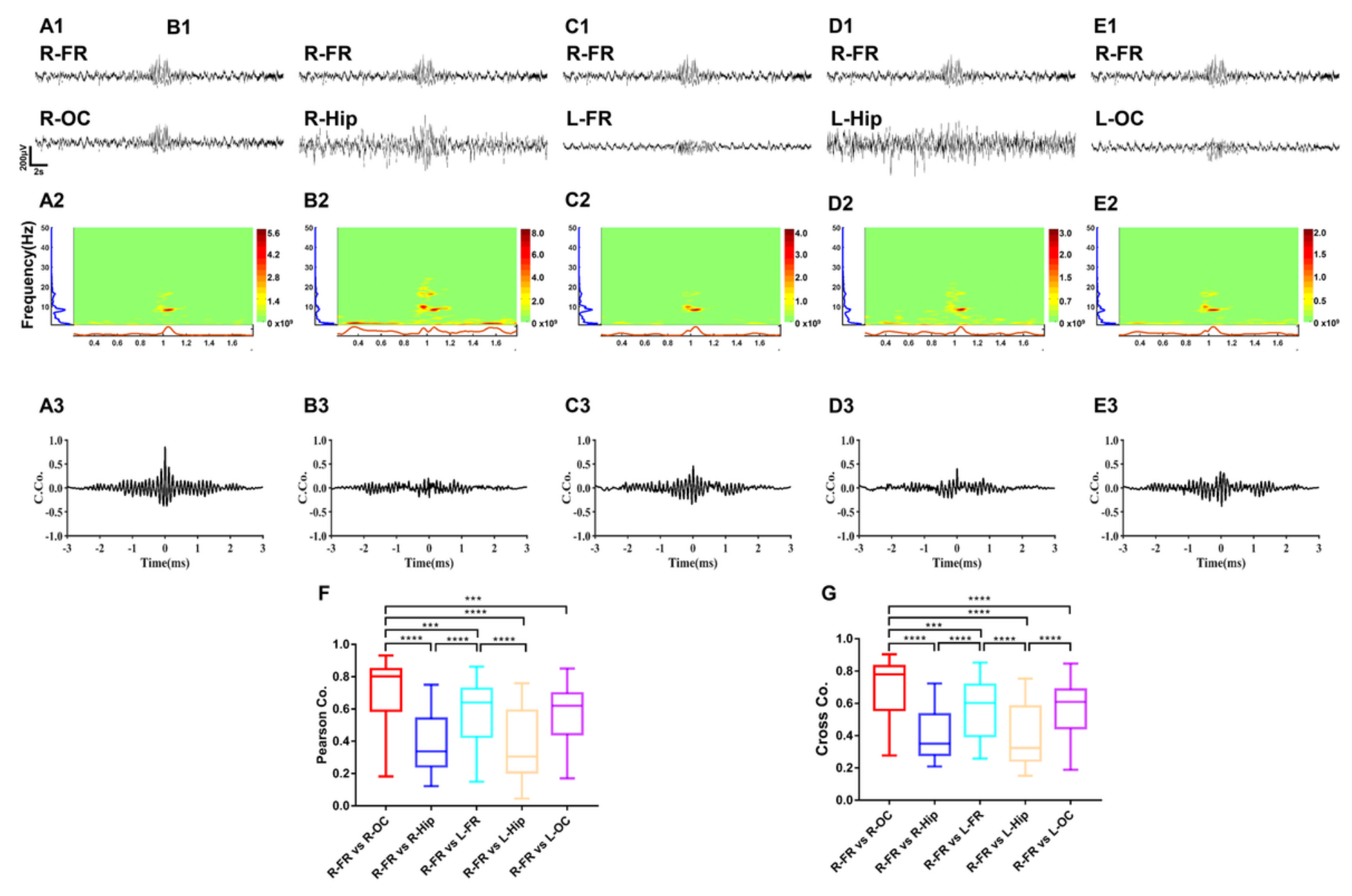

\section{Figure 7}

The synchrony degree of spontaneous discharges between different brain regions. The results showed the right frontal cortex and occipital cortex had the highest degree of synchrony(A1-G). Intriguing, synchronization of spontaneous spike-wave discharges was concentrated to $8 \mathrm{~Hz}$ (A2-E2). The coherence and cross-correlation analysis also showed right frontal cortex and occipital cortex had the maximum coefficient, secondly the coefficient between right frontal cortex and left frontal cortex(A3-G). Nonetheless, the spontaneous spike-wave discharges between cortex and hippocampus displayed various degree of

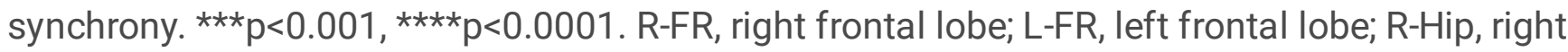
hippocampus; L-Hip, left hippocampus; R-OC, right occipital lobe; L-OC, left occipital lobe. 


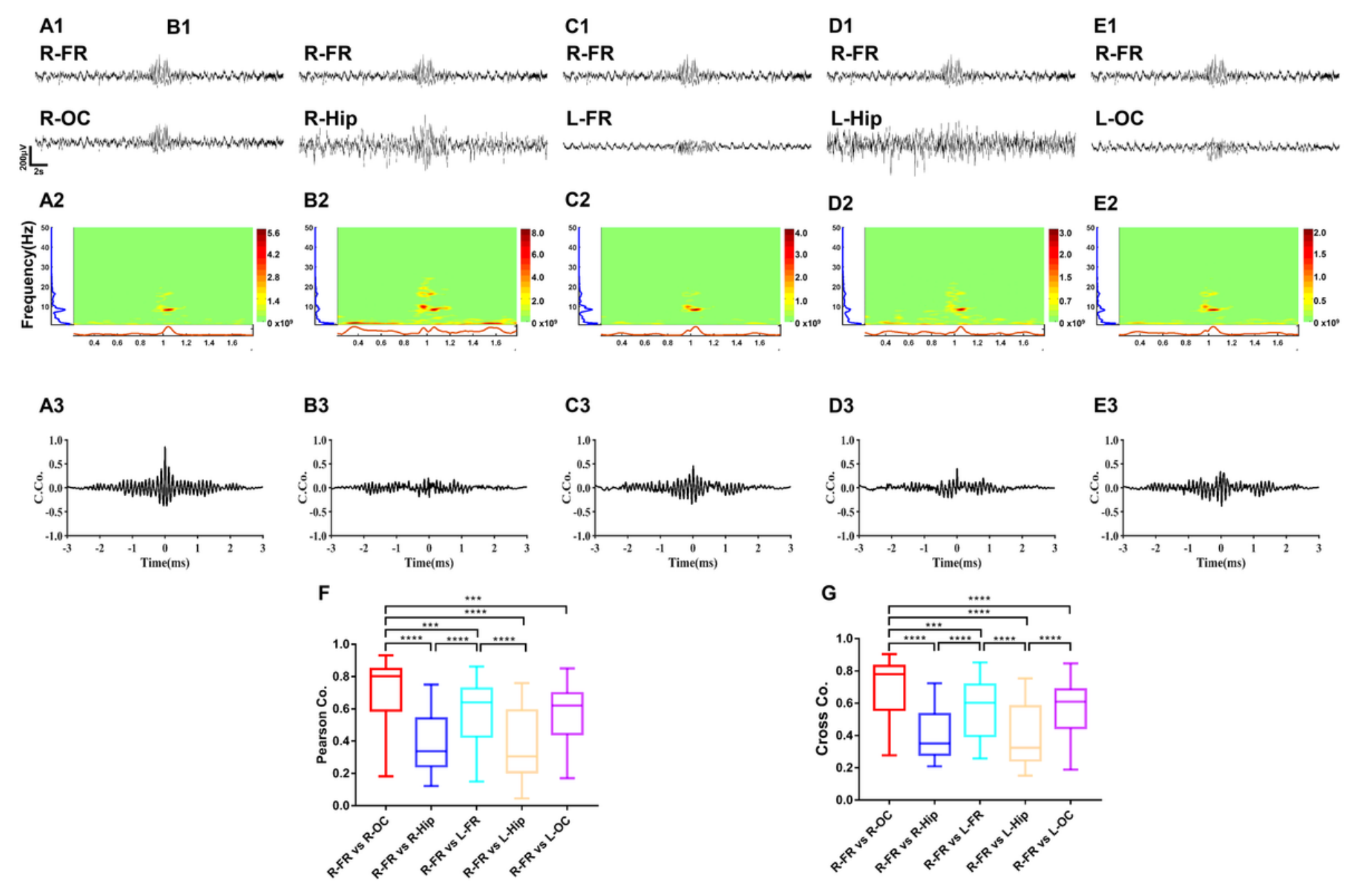

\section{Figure 7}

The synchrony degree of spontaneous discharges between different brain regions. The results showed the right frontal cortex and occipital cortex had the highest degree of synchrony(A1-G). Intriguing, synchronization of spontaneous spike-wave discharges was concentrated to $8 \mathrm{~Hz}$ (A2-E2). The coherence and cross-correlation analysis also showed right frontal cortex and occipital cortex had the maximum coefficient, secondly the coefficient between right frontal cortex and left frontal cortex(A3-G). Nonetheless, the spontaneous spike-wave discharges between cortex and hippocampus displayed various degree of

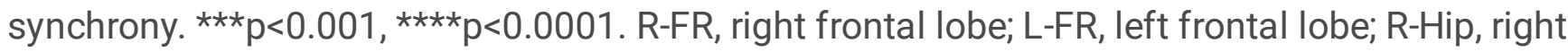
hippocampus; L-Hip, left hippocampus; R-OC, right occipital lobe; L-OC, left occipital lobe. 


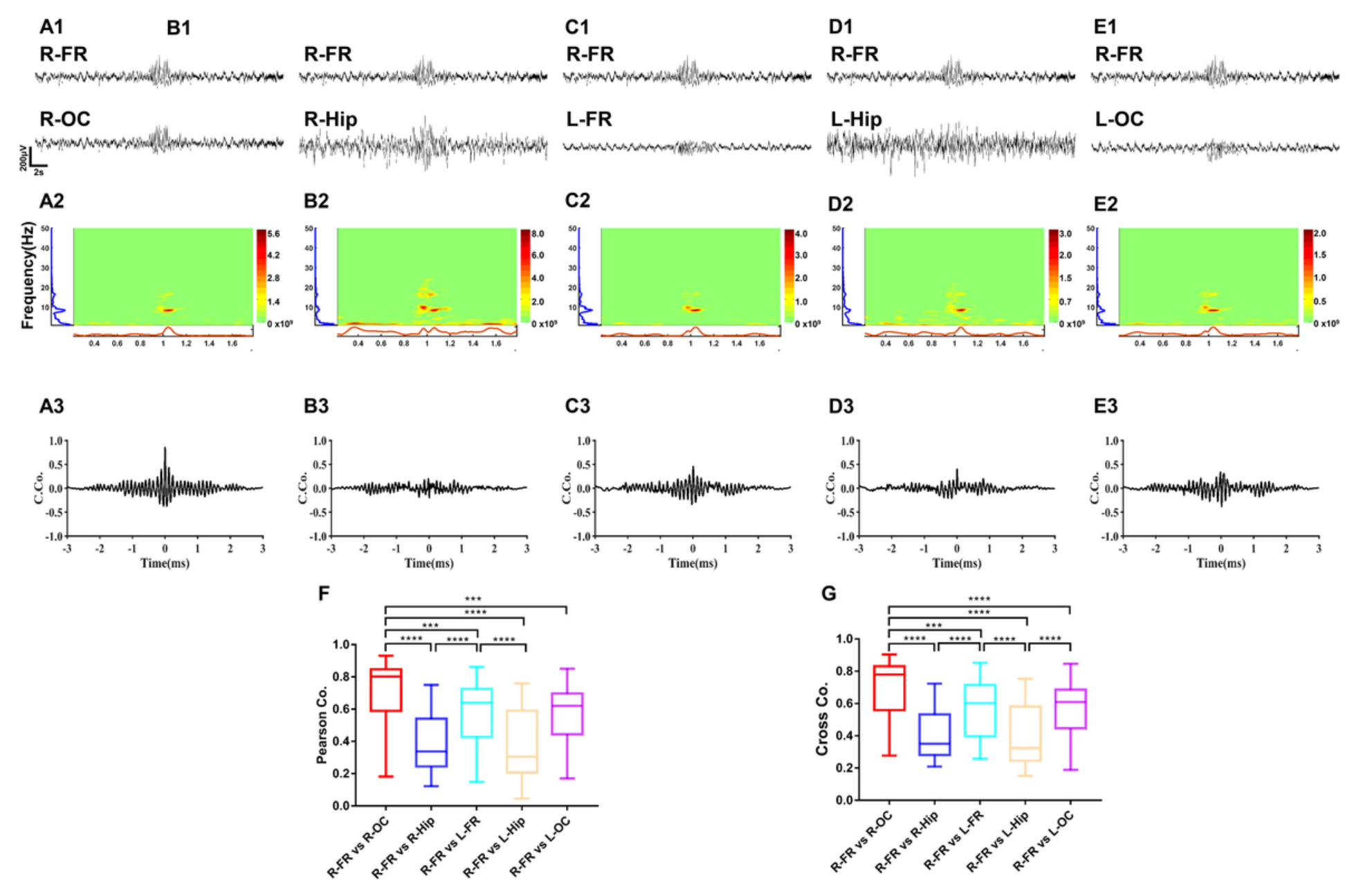

\section{Figure 7}

The synchrony degree of spontaneous discharges between different brain regions. The results showed the right frontal cortex and occipital cortex had the highest degree of synchrony(A1-G). Intriguing, synchronization of spontaneous spike-wave discharges was concentrated to $8 \mathrm{~Hz}$ (A2-E2). The coherence and cross-correlation analysis also showed right frontal cortex and occipital cortex had the maximum coefficient, secondly the coefficient between right frontal cortex and left frontal cortex(A3-G). Nonetheless, the spontaneous spike-wave discharges between cortex and hippocampus displayed various degree of

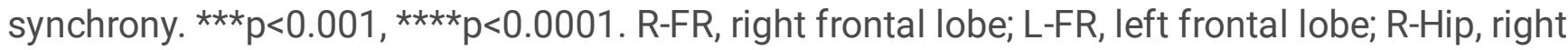
hippocampus; L-Hip, left hippocampus; R-OC, right occipital lobe; L-OC, left occipital lobe. 
A

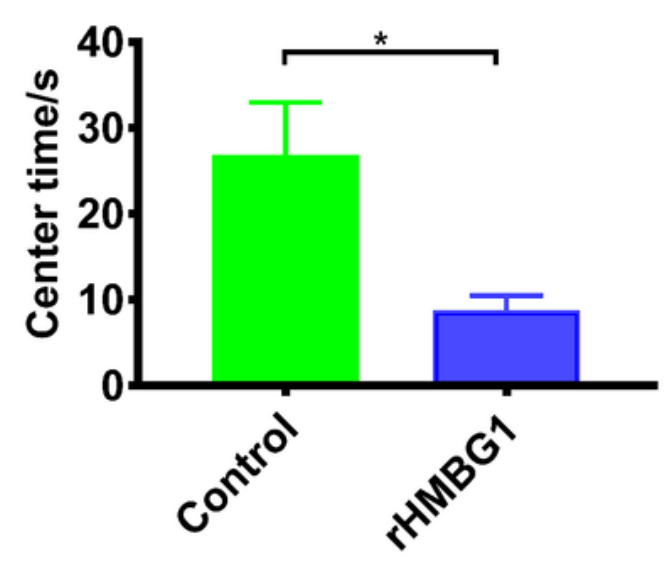

C

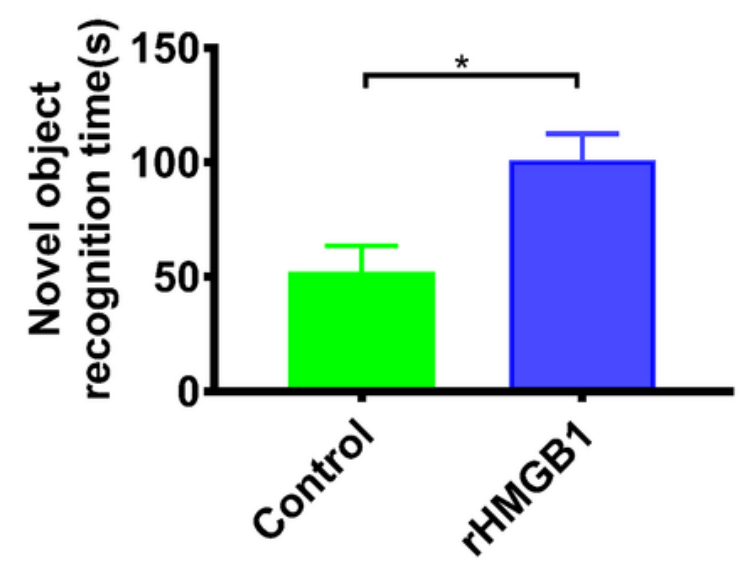

E

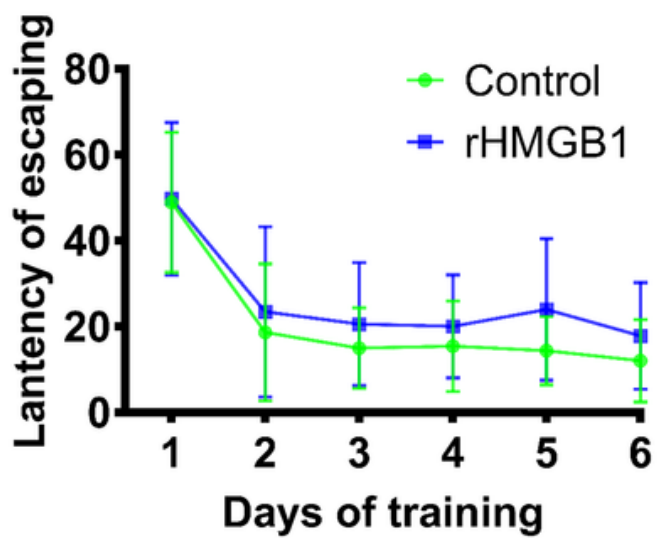

B

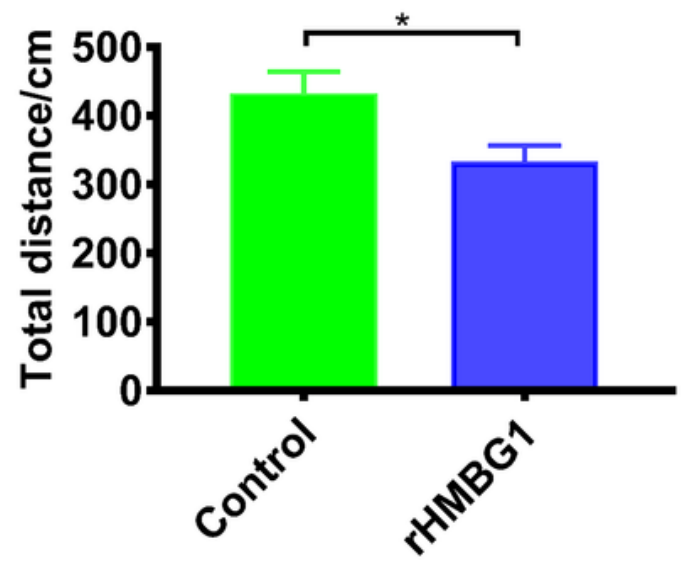

D

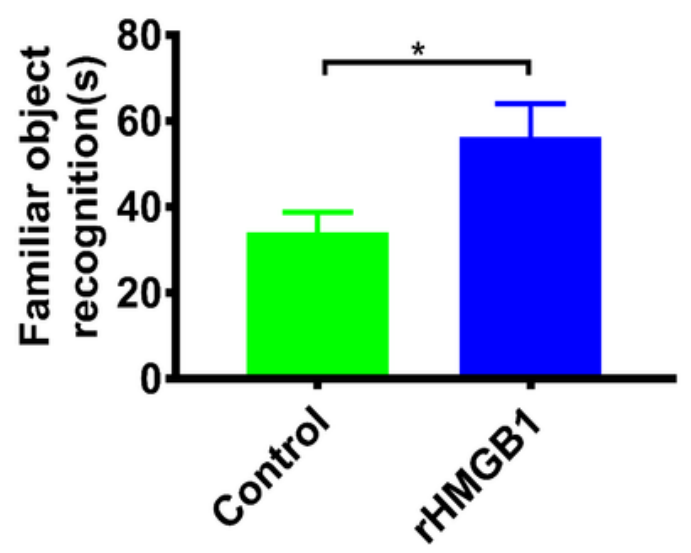

$\mathbf{F}$

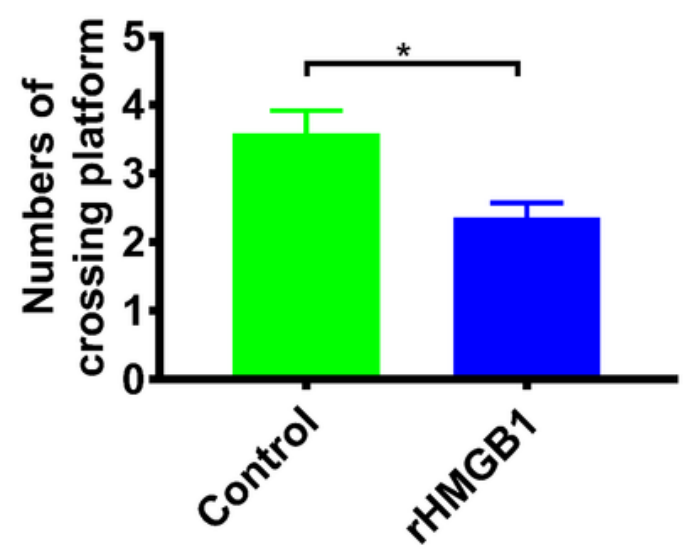

Figure 8

Rats displayed impaired cognition and spatial learning and memory in rHMGB1-treatment group. A-B: The center time and total activity distance. C-D: Average time exploring novel and familiar object during 5 min test. E-F: The escape latency during training days and the numbers of crossing platform during $10 \mathrm{~min}$ probe test. ${ }^{*} p<0.05$. rHMGB1, rHMGB1-treatment 
A

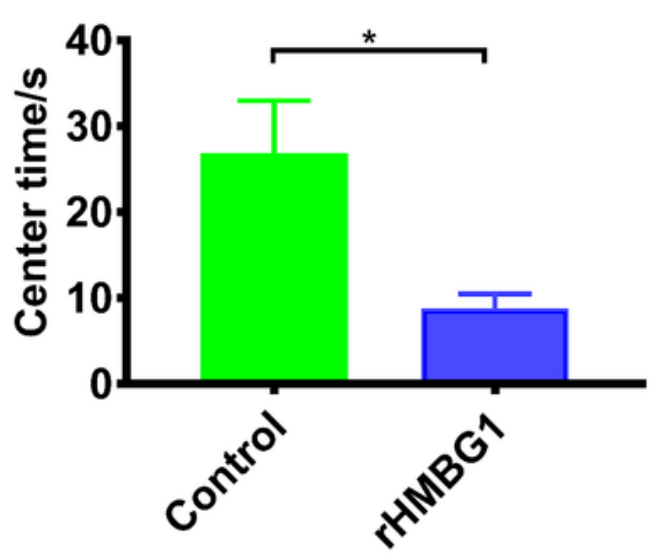

C

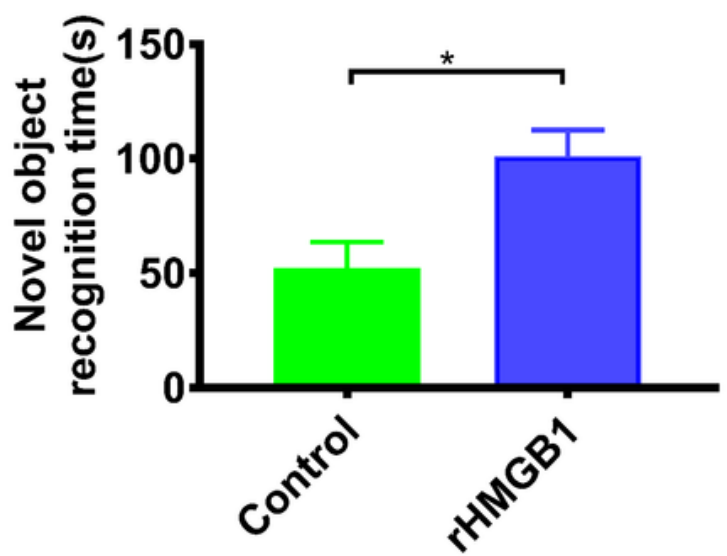

$\mathbf{E}$

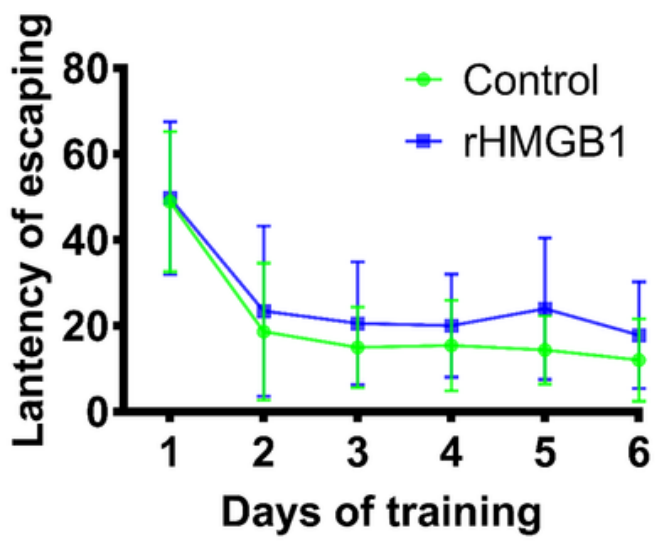

B

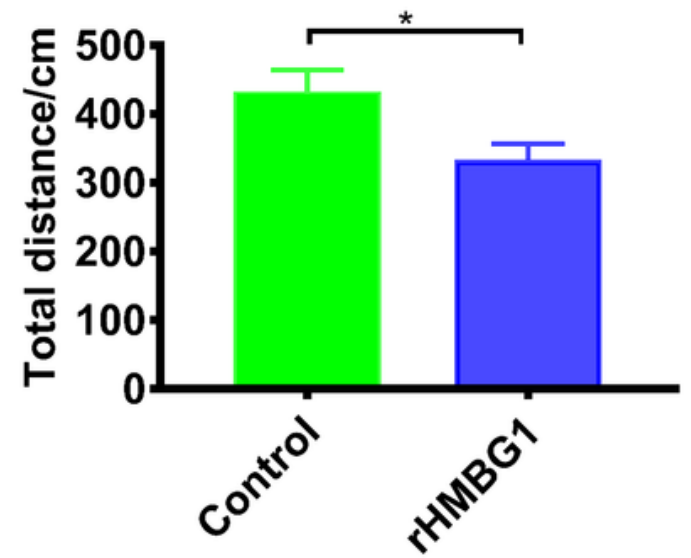

D

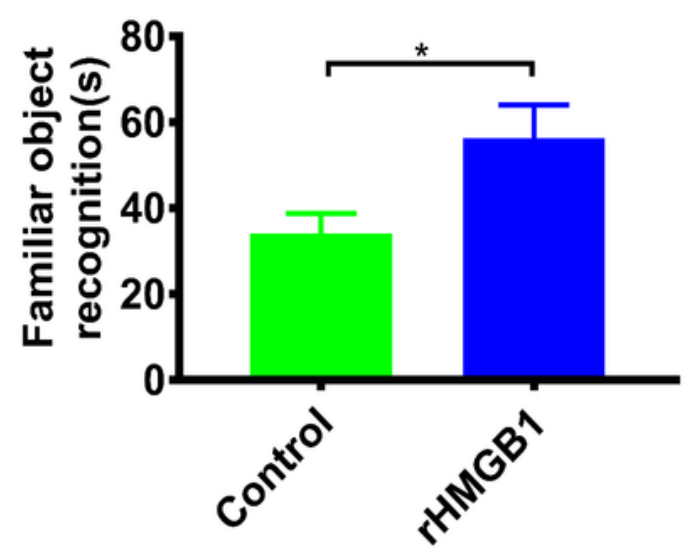

F

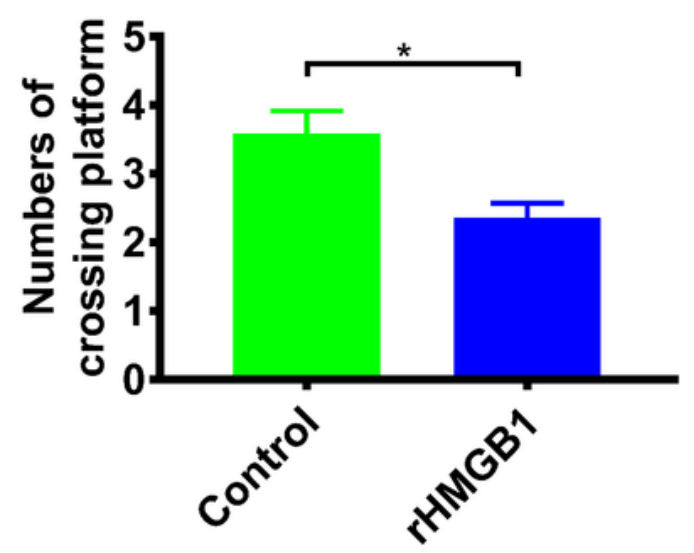

Figure 8

Rats displayed impaired cognition and spatial learning and memory in rHMGB1-treatment group. A-B: The center time and total activity distance. C-D: Average time exploring novel and familiar object during 5 min test. E-F: The escape latency during training days and the numbers of crossing platform during $10 \mathrm{~min}$ probe test. ${ }^{*} \mathrm{p}<0.05$. rHMGB1, rHMGB1-treatment 
A

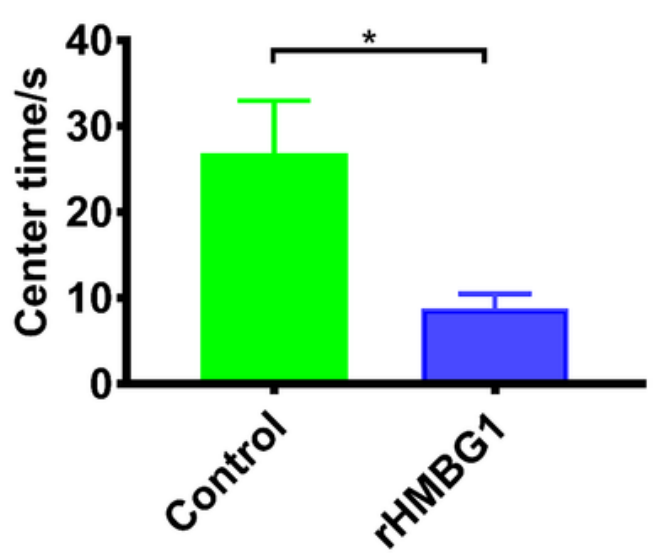

C

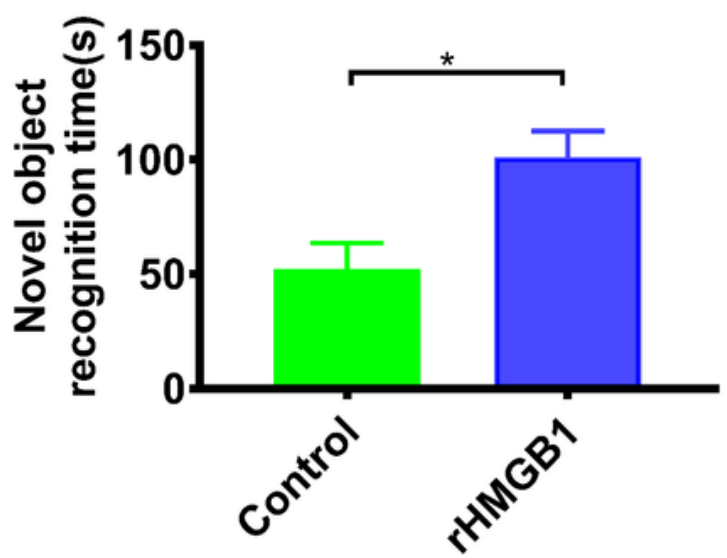

$\mathbf{E}$

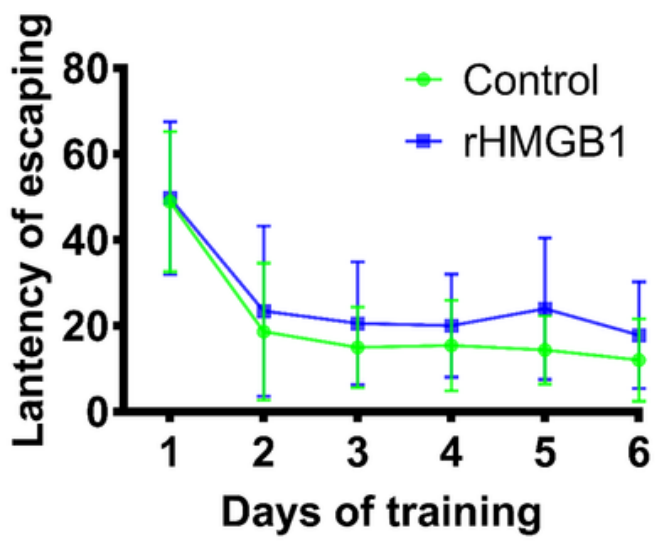

B

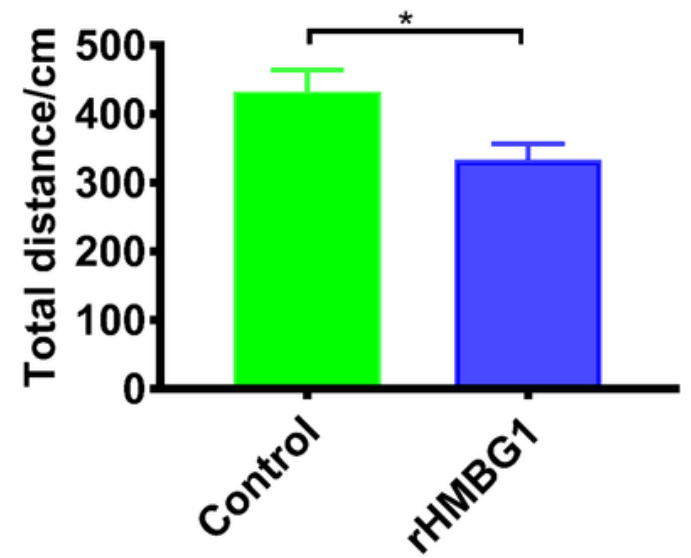

D

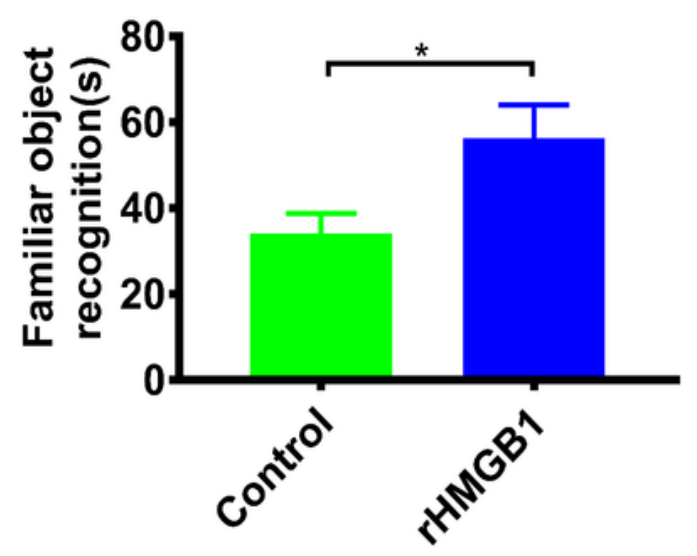

F

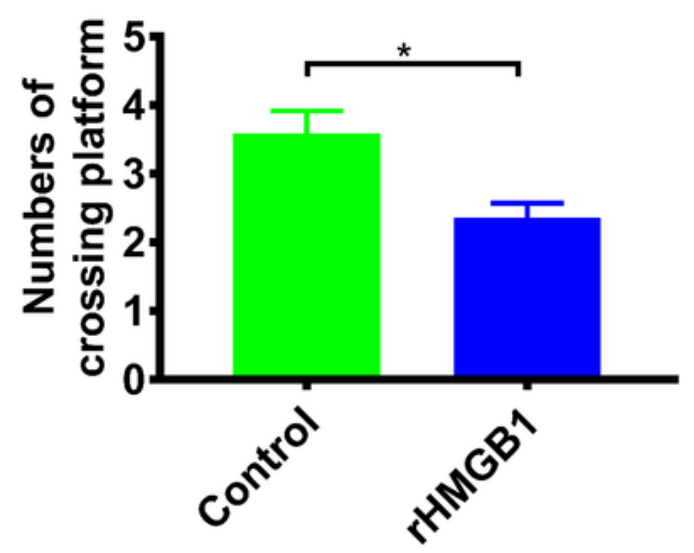

Figure 8

Rats displayed impaired cognition and spatial learning and memory in rHMGB1-treatment group. A-B: The center time and total activity distance. C-D: Average time exploring novel and familiar object during 5 min test. E-F: The escape latency during training days and the numbers of crossing platform during $10 \mathrm{~min}$ probe test. ${ }^{*} \mathrm{p}<0.05$. rHMGB1, rHMGB1-treatment 
A

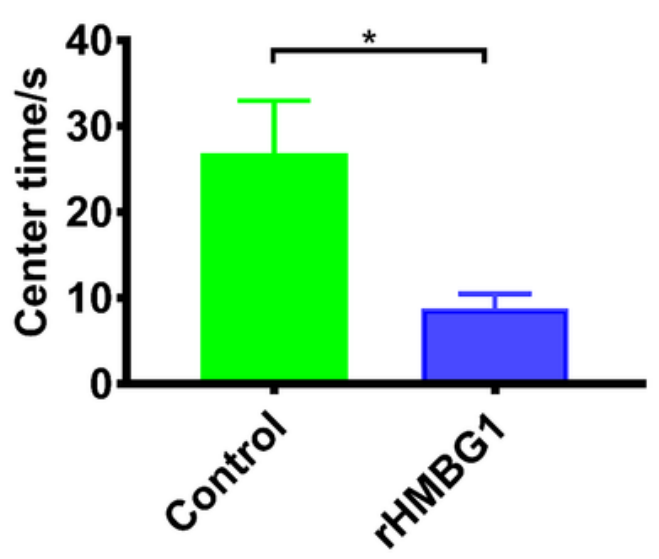

C

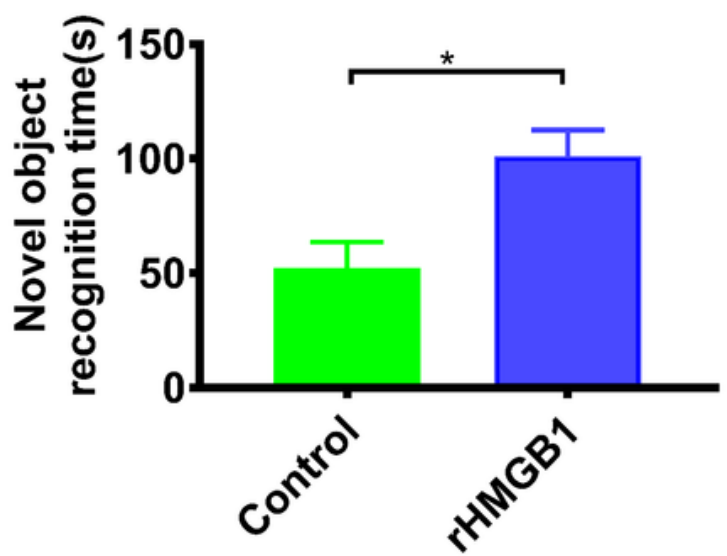

$\mathbf{E}$

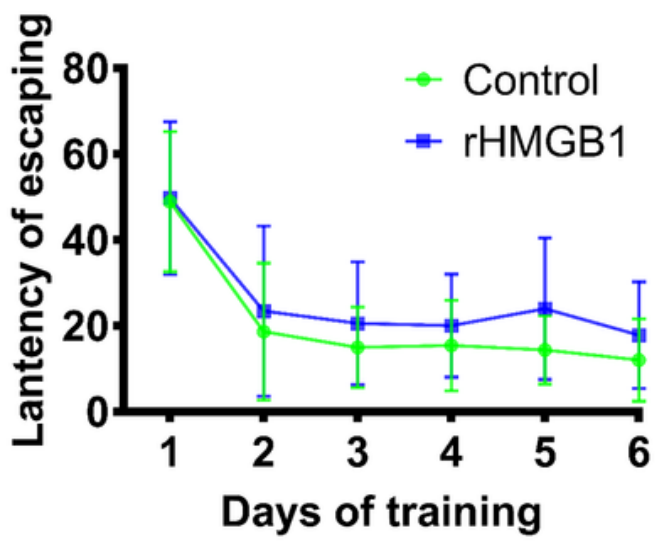

B

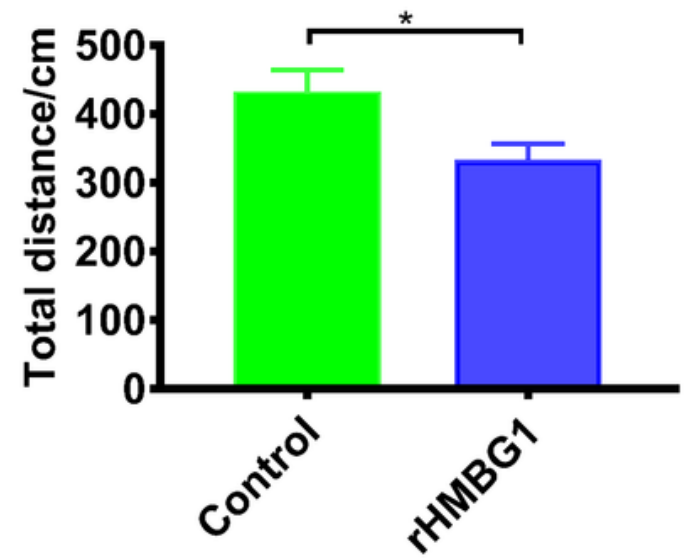

D

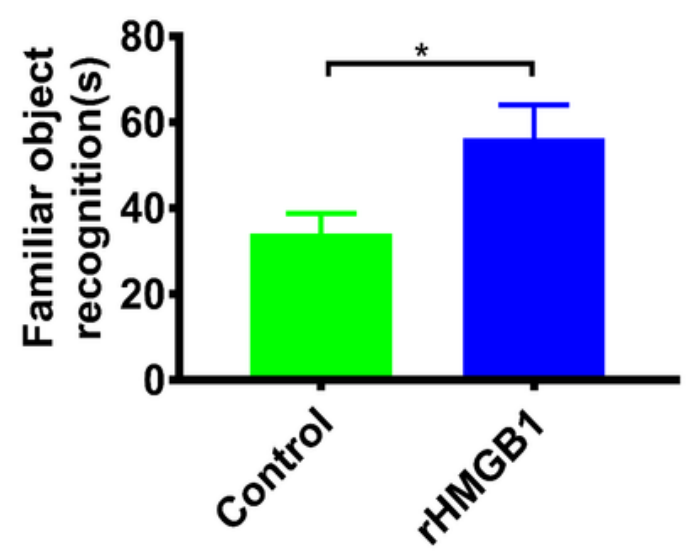

F

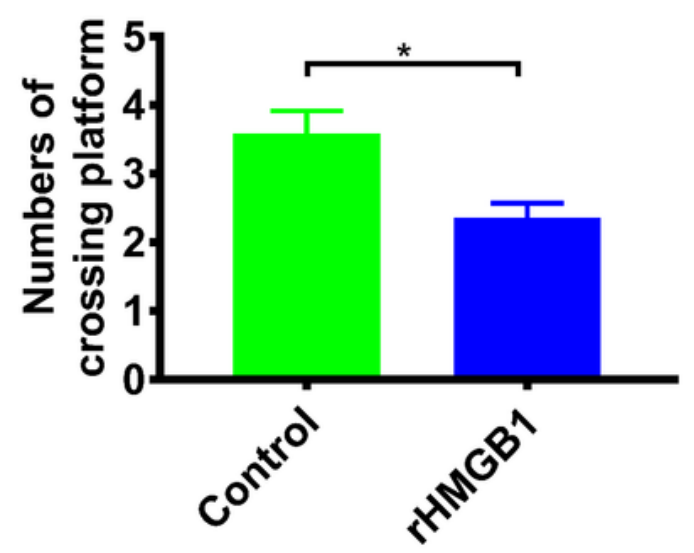

Figure 8

Rats displayed impaired cognition and spatial learning and memory in rHMGB1-treatment group. A-B: The center time and total activity distance. C-D: Average time exploring novel and familiar object during 5 min test. E-F: The escape latency during training days and the numbers of crossing platform during $10 \mathrm{~min}$ probe test. ${ }^{*} \mathrm{p}<0.05$. rHMGB1, rHMGB1-treatment 